\title{
Bioactive Secondary Metabolites of the Genus Diaporthe and Anamorph Phomopsis from Terrestrial and Marine Habitats and Endophytes: 2010-2019
}

\author{
Tang-Chang Xu, Yi-Han Lu, Jun-Fei Wang, Zhi-Qiang Song, Ya-Ge Hou, Si-Si Liu, Chuan-Sheng Liu and \\ Shao-Hua $\mathrm{Wu} *$ (i)
}

\section{check for} updates

Citation: Xu, T.-C.; Lu, Y.-H.; Wang, J.-F.; Song, Z.-Q.; Hou, Y.-G.; Liu, S.-S.; Liu, C.-S.; Wu, S.-H. Bioactive Secondary Metabolites of the Genus Diaporthe and Anamorph Phomopsis from Terrestrial and Marine Habitats and Endophytes: 2010-2019. Microorganisms 2021, 9, 217. https://doi.org/10.3390/ microorganisms 9020217

\section{Academic Editor:}

Carlos García-Estrada

Received: 31 December 2020

Accepted: 18 January 2021

Published: 21 January 2021

Publisher's Note: MDPI stays neutral with regard to jurisdictional claims in published maps and institutional affiliations.

Copyright: (c) 2021 by the authors. Licensee MDPI, Basel, Switzerland. This article is an open access article distributed under the terms and conditions of the Creative Commons Attribution (CC BY) license (https:/ / creativecommons.org/licenses/by/ $4.0 /)$.
Yunnan Institute of Microbiology, School of Life Sciences, Yunnan University, Kunming 650091, China; xu2950129@163.com (T.-C.X.); luyihan1995@126.com (Y.-H.L.); wang_junfei@163.com (J.-F.W.); songzhiqiang1996@126.com (Z.-Q.S.); houyage@126.com (Y.-G.H.); liusisi1994@126.com (S.-S.L.); liucs313@126.com (C.-S.L.)

* Correspondence: shwu123@126.com

\begin{abstract}
The genus Diaporthe and its anamorph Phomopsis are distributed worldwide in many ecosystems. They are regarded as potential sources for producing diverse bioactive metabolites. Most species are attributed to plant pathogens, non-pathogenic endophytes, or saprobes in terrestrial host plants. They colonize in the early parasitic tissue of plants, provide a variety of nutrients in the cycle of parasitism and saprophytism, and participate in the basic metabolic process of plants. In the past ten years, many studies have been focused on the discovery of new species and biological secondary metabolites from this genus. In this review, we summarize a total of 335 bioactive secondary metabolites isolated from 26 known species and various unidentified species of Diaporthe and Phomopsis during 2010-2019. Overall, there are 106 bioactive compounds derived from Diaporthe and 246 from Phomopsis, while 17 compounds are found in both of them. They are classified into polyketides, terpenoids, steroids, macrolides, ten-membered lactones, alkaloids, flavonoids, and fatty acids. Polyketides constitute the main chemical population, accounting for $64 \%$. Meanwhile, their bioactivities mainly involve cytotoxic, antifungal, antibacterial, antiviral, antioxidant, antiinflammatory, anti-algae, phytotoxic, and enzyme inhibitory activities. Diaporthe and Phomopsis exhibit their potent talents in the discovery of small molecules for drug candidates.
\end{abstract}

Keywords: ascomycetes; endophytic fungi; plant pathogens; biological activities; natural products

\section{Introduction}

Diaporthe is an important fungal genus of plant pathogens [1] belonging to the family Diaporthaceae, order Diaporthales, class Sordariomycetes [2]. It is mainly isolated from various hosts distributed in tropical and temperate zones and can cause diseases to a wide range of plant hosts, as well as humans and other mammals [3,4]. The ascomycetes of Diaporthe Nitschke 1870 and Phomopsis (Sacc.) Bubák 1905 are regarded to form a genus [5,6]. In Index Fungorum (2020), more than 1120 records of Diaporthe and 986 of Phomopsis are listed (http:/ / www.indexfungorum.org/, accessed December 2020). There is a common understanding that, in these ascomycetes, the teleomorph states are named as Diaporthe and the anamorph states called as Phomopsis [7-10]. For a long time, a dispute has remained concerning whether the generic name should be defined as Diaporthe or Phomopsis. Due to the importance of this genus as plant pathogens, the classification of Diaporthe has been discussed by many researchers. Since Diaporthe was cited earlier and represents most of the species described in nature, more mycologists suggest that the use of Diaporthe as a generic name have more priority and is more suitable for the current study of this fungal group [11-13]. In recent years, the previous classification methods based on morphological characteristics are no longer applicable to the genus Diaporthe and advanced molecular 
techniques will replace them to solve the classification problem of Diaporthe $[13,14]$. In this review, we use the older name Diaporthe as the generic name.

Based on the existing literature investigations, more secondary metabolites have been separated from Phomopsis than Diaporthe. To date, a large number of compounds have been isolated from endophytic fungi of terrestrial plants in Diaporthe and Phomopsis, some of which originate from the marine environment (mainly mangroves and sediments). Most of compounds are classified as polyketides, which is the main structural type of secondary metabolites in this genus. The reported compounds showed various bioactivities, such as cytotoxic [15], antifungal [16], antibacterial [17], antiviral [18], antioxidant [19], antiinflammatory [20], phytotoxic [21], and enzyme inhibition [22]. Up to now, there are 26 known species and various unidentified species of Diaporthe and Phomopsis have been studied for their metabolites. Our current review comprehensively summarize a total of 335 bioactive natural products from Diaporthe and Phomopsis between 2010 and 2019, covering their detailed chemical structures with classifications in structural types, as well as their bioactivities and habitats.

\section{Bioactive Secondary Metabolites from Phomopsis}

The Phomopsis fungi are important resource of bioactive compounds in the field of drug discovery, and have remarkable medical application value. According to the literature reports in recent ten years, a total of 246 bioactive compounds are summarized from Phomopsis herein. These substances have rich and diverse biological activities, such as cytotoxic, antifungal, antibacterial, antiviral, antioxidant, anti-inflammatory, phytotoxic, antimalarial, antialgae, antimigratory, pro-apoptotic, accelerating, and inhibiting the growth of subintestinal vessel plexus (SIV) branches, protecting effects on pancreatic $\beta$-cells, motility inhibitory and zoosporicidal potential, and enzyme inhibitory activities (Table 1). Among them, some interesting and promising bioactive compounds might be used in pharmaceutical and agricultural fields. The derived habitats of the Phomopsis strains can also be found in Table 1, which shows that there are 174 (accounting for $71 \%$ ) and 66 (accounting for $27 \%$ ) compounds obtained from terrestrial and marine environments, respectively, while six compounds (accounting for $2 \%$ ) were not mentioned their habitats.

Table 1. The bioactive secondary metabolites of the anamorph Phomopsis during 2010-2019.

\begin{tabular}{|c|c|c|c|c|c|c|}
\hline Number & Structural Types & Compounds & Strains & $\begin{array}{l}\text { Habitats } \\
\left(\mathrm{T} / \mathrm{M}^{\mathrm{a}}\right)\end{array}$ & Activities & Refs. \\
\hline 1 & Xanthones & $\begin{array}{l}\text { 1,5-Dihydroxy-3- } \\
\text { hydroxyethyl-6-methoxy- } \\
\text { carbonylxanthone }\end{array}$ & Phomopsis sp. & $\begin{array}{l}\text { Paris polyphylla } \\
\text { var. yunnanensis } \\
\text { (T) }\end{array}$ & Cytotoxic & [23] \\
\hline 2 & & $\begin{array}{l}\text { 1-Hydroxy-5-methoxy-3- } \\
\text { hydroxyethyl-6- } \\
\text { methoxycarbonylxanthone }\end{array}$ & Phomopsis sp. & $\begin{array}{l}\text { P. polyphylla var. } \\
\text { yunnanensis }(\mathrm{T})\end{array}$ & Cytotoxic & [23] \\
\hline 3 & & $\begin{array}{l}\text { 1-Hydroxy-3-hydroxyethyl-8- } \\
\text { ethoxycarbonyl-xanthone }\end{array}$ & Phomopsis sp. & $\begin{array}{l}\text { P. polyphylla var. } \\
\text { yunnanensis }(\mathrm{T})\end{array}$ & Cytotoxic & [23] \\
\hline 4 & & Pinselin & Phomopsis sp. & $\begin{array}{l}\text { P. polyphylla var. } \\
\text { yunnanensis }(\mathrm{T})\end{array}$ & Cytotoxic & [23] \\
\hline 5 & & $\begin{array}{l}\text { 1-Hydroxy-8- } \\
\text { (hydroxymethyl)-3-methoxy- } \\
\text { 6-methylxanthone }\end{array}$ & Phomopsis sp. & $\begin{array}{l}\text { P. polyphylla var. } \\
\text { yunnanensis }(\mathrm{T})\end{array}$ & Cytotoxic & [23] \\
\hline 6 & & $\begin{array}{c}\text { 2,6-Dihydroxy-3-methyl-9- } \\
\text { oxoxanthene-8-carboxylic acid } \\
\text { methyl ester }\end{array}$ & $\begin{array}{l}\text { Phomopsis sp. (No. } \\
\text { SK7RN3G1) }\end{array}$ & Sediment (M) & Cytotoxic & [24] \\
\hline 7 & & $\begin{array}{c}\text { 4,5-Dihydroxy-3-(2- } \\
\text { hydroxyethyl)-1-methoxy-8- } \\
\text { methoxy- } \\
\text { carbonylxanthone }\end{array}$ & P. amygdali & Paris axialis $(\mathrm{T})$ & Cytotoxic & [25] \\
\hline 8 & & $\begin{array}{c}\text { 1,8-Dihydroxy-4-(2- } \\
\text { hydroxyethyl)-3- } \\
\text { methoxyxanthone }\end{array}$ & P. amygdali & P. axialis $(\mathrm{T})$ & Cytotoxic & [25] \\
\hline 9 & & Hydroxyvertixanthone & $\begin{array}{c}\text { Phomopsis sp. YM } \\
355364\end{array}$ & $\begin{array}{c}\text { Aconitum } \\
\text { carmichaelii }(\mathrm{T})\end{array}$ & Antimicrobial & [26] \\
\hline
\end{tabular}


Table 1. Cont.

\begin{tabular}{|c|c|c|c|c|c|c|}
\hline Number & Structural Types & Compounds & Strains & $\begin{array}{l}\text { Habitats } \\
\left(\mathrm{T} / \mathbf{M}^{\mathrm{a}}\right)\end{array}$ & Activities & Refs. \\
\hline 10 & & Dalienxanthone A & Phomopsis sp. & Paris daliensis $(\mathrm{T})$ & Cytotoxic & [27] \\
\hline 11 & & Dalienxanthone B & Phomopsis sp. & P. daliensis $(\mathrm{T})$ & Cytotoxic & [27] \\
\hline 12 & & Dalienxanthone C & Phomopsis sp. & P. daliensis $(\mathrm{T})$ & Cytotoxic & [27] \\
\hline 13 & & Paucinervin E & P. amygdali & P. axialis $(\mathrm{T})$ & Cytotoxic & [25] \\
\hline 14 & & $\begin{array}{l}\text { 1,3-Dihydroxy-4-(1,3,4- } \\
\text { trihydroxybutan-2-yl)-8- } \\
\text { methoxy-9H-xanthen-9-one }\end{array}$ & P. amygdali & $\begin{array}{l}\text { P. polyphylla var. } \\
\text { yunnanensis }(\mathrm{T})\end{array}$ & Cytotoxic & [28] \\
\hline 15 & & $\begin{array}{l}\text { 3-Methoxy-1,4,8-trihydroxy-5- } \\
\left(1^{\prime}, 3^{\prime}, 4^{\prime} \text {-trihydroxybutan-2'- }\right. \\
\text { yl)-xanthone }\end{array}$ & P. amygdali & P.axialis $(\mathrm{T})$ & Cytotoxic & [29] \\
\hline 16 & & $\begin{array}{c}\text { 8-Methoxy-1,3,4-trihydroxy-5- } \\
\left(1^{\prime}, 3^{\prime}, 4^{\prime} \text {-trihydroxybutan-2'- }\right. \\
\text { yl)-xanthone }\end{array}$ & P. amygdali & P.axialis $(\mathrm{T})$ & Cytotoxic & [29] \\
\hline 17 & & Secosterigmatocystin & $\begin{array}{l}\text { Phomopsis sp. } \\
\text { P. amygdali }\end{array}$ & $\begin{array}{l}\text { P. polyphylla var. } \\
\text { yunnanensis }(\mathrm{T}) \\
\text { P.axialis }(\mathrm{T})\end{array}$ & $\begin{array}{l}\text { Cytotoxic } \\
\text { Cytotoxic }\end{array}$ & $\begin{array}{l}{[23]} \\
{[29]}\end{array}$ \\
\hline 18 & & $\begin{array}{l}\text { 3,8-Dihydroxy-4-(2,3- } \\
\text { dihydroxy-1- } \\
\text { hydroxymethylpropyl)-1- } \\
\text { methoxyxanthone }\end{array}$ & Phomopsis sp. & P. daliensis $(\mathrm{T})$ & Cytotoxic & [27] \\
\hline 19 & & Oliganthins E & Phomopsis sp. & P. daliensis $(\mathrm{T})$ & Cytotoxic & [27] \\
\hline 20 & & Dihydrosterigmatocystin & P. amygdali & P.axialis $(\mathrm{T})$ & Cytotoxic & [29] \\
\hline 21 & & Vieillardixanthone & P. amygdali & P. axialis (T) & Cytotoxic & [29] \\
\hline 22 & & $\begin{array}{l}\text { 1,7-Dihydroxy-2-methoxy-3- } \\
\text { (3-methylbut-2-enyl)xanthone }\end{array}$ & Phomopsis sp. & $\begin{array}{l}\text { P. polyphylla var. } \\
\text { yunnanensis }(\mathrm{T})\end{array}$ & Cytotoxic & [23] \\
\hline 23 & & $\begin{array}{l}\text { 1-Hydroxy-4,7-dimethoxy-6- } \\
\text { (3-oxobutyl)-xanthone }\end{array}$ & Phomopsis sp. & $\begin{array}{l}\text { P. polyphylla var. } \\
\text { yunnanensis }(\mathrm{T})\end{array}$ & Cytotoxic & [23] \\
\hline 24 & & Asperxanthone & Phomopsis sp. & $\begin{array}{l}\text { P. polyphylla var. } \\
\text { yunnanensis }(\mathrm{T})\end{array}$ & Cytotoxic & [23] \\
\hline 25 & & $\begin{array}{c}\text { 6-O-Methyl-2- } \\
\text { deprenylrheediaxanthone } \\
\text { B }\end{array}$ & Phomopsis sp. & $\begin{array}{l}\text { P. polyphylla var. } \\
\text { yunnanensis }(\mathrm{T})\end{array}$ & Cytotoxic & [23] \\
\hline 26 & & $\begin{array}{l}\text { Cratoxylumxanthone D } \\
\text { 3-O-(6-O- } \alpha \text {-L- }\end{array}$ & Phomopsis sp. & P. daliensis $(\mathrm{T})$ & Cytotoxic & [27] \\
\hline 27 & & $\begin{array}{l}\text { Arabinopyranosyl)- } \beta \text {-D- } \\
\text { glucopyranosyl-1,4- } \\
\text { dimethoxyxanthone }\end{array}$ & $\begin{array}{l}\text { Phomopsis sp. } \\
\quad(\text { ZH76) }\end{array}$ & $\begin{array}{c}\text { Excoecaria } \\
\text { agallocha }(\mathrm{M})\end{array}$ & Cytotoxic & [30] \\
\hline 28 & & Phomoxanthone A & $\begin{array}{c}\text { P. longicolla } \\
\text { Phomopsis sp. IM } \\
41-1 \\
\text { Phomopsis sp. 33\# }\end{array}$ & $\begin{array}{c}\text { Sonneratia } \\
\text { caseolaris }(\mathrm{M}) \\
\text { Rhizhopora } \\
\text { mucronata }(\mathrm{M}) \\
\text { Rhizophora stylosa } \\
\text { (M) }\end{array}$ & $\begin{array}{l}\text { Pro-apoptotic } \\
\text { Antimicrobial } \\
\text { Inhibiting } \\
\text { acetylcholinesterase } \\
\text { and } \alpha \text {-glucosidase, } \\
\text { Antioxidant }\end{array}$ & $\begin{array}{l}{[31]} \\
{[32]} \\
{[33]}\end{array}$ \\
\hline 29 & & $\begin{array}{l}\text { 12-O-Deacetyl- } \\
\text { phomoxanthone } \\
\text { A }\end{array}$ & $\begin{array}{l}\text { Phomopsis sp. IM } \\
\quad 41-1\end{array}$ & R. mucronata $(\mathrm{M})$ & Antimicrobial & [32] \\
\hline 30 & & Dicerandrol A & $\begin{array}{l}\text { P. longicolla S1B4 } \\
\text { Phomopsis sp. } \\
\text { HNY29-2B }\end{array}$ & $\begin{array}{c}-\mathrm{b} \\
\text { Acanthus ilicifolius } \\
\text { (M) }\end{array}$ & $\begin{array}{l}\text { Antimicrobial } \\
\text { Cytotoxic }\end{array}$ & $\begin{array}{l}{[34]} \\
{[35]}\end{array}$ \\
\hline 31 & & Dicerandrol B & $\begin{array}{l}\text { P. longicolla S1B4 } \\
\text { Phomopsis sp. } \\
\text { HNY29-2B }\end{array}$ & $\stackrel{-\mathrm{b}}{\text { A. ilicifolius (M) }}$ & $\begin{array}{l}\text { Antibacterial } \\
\text { Cytotoxic }\end{array}$ & {$[34]$} \\
\hline 32 & & Dicerandrol C & P. longicolla S1B4 & $-b$ & Antibacterial & [34] \\
\hline 33 & & Deacetylphomoxanthone B & $\begin{array}{l}\text { P. longicolla S1B4 } \\
\text { Phomopsis sp. } \\
\text { HNY29-2B }\end{array}$ & A. ilicifolius(M) & $\begin{array}{l}\text { Antibacterial } \\
\text { Cytotoxic }\end{array}$ & $\begin{array}{l}{[34]} \\
{[35]}\end{array}$ \\
\hline 34 & & Penexanthone A & $\begin{array}{l}\text { Phomopsis sp. } \\
\text { HNY29-2B }\end{array}$ & A. ilicifolius (M) & Cytotoxic & [35] \\
\hline 35 & Chromones & (+)-Phomopsichin A & Phomopsis sp. 33\# & R. stylosa (M) & $\begin{array}{l}\text { Antimicrobial, } \\
\text { Antioxidant, } \\
\text { Inhibiting } \\
\text { acetylcholinesterase } \\
\text { and } \alpha \text {-glucosidase }\end{array}$ & [33] \\
\hline
\end{tabular}


Table 1. Cont.

\begin{tabular}{|c|c|c|c|c|c|c|}
\hline Number & Structural Types & Compounds & Strains & $\begin{array}{l}\text { Habitats } \\
\left(\mathrm{T} / \mathrm{M}^{\mathrm{a}}\right)\end{array}$ & Activities & Refs. \\
\hline 36 & & (-)-Phomopsichin B & Phomopsis sp. 33\# & R. stylosa (M) & $\begin{array}{c}\text { Antimicrobial, } \\
\text { Antioxidant, } \\
\text { Inhibiting } \\
\text { acetylcholinesterase } \\
\text { and } \alpha \text {-glucosidase }\end{array}$ & [33] \\
\hline 37 & & Phomopsichin C & Phomopsis sp. 33\# & R. stylosa (M) & $\begin{array}{l}\text { Antimicrobial, } \\
\text { Antioxidant, } \\
\text { Inhibiting } \\
\text { acetylcholinesterase } \\
\text { and } \alpha \text {-glucosidase }\end{array}$ & [33] \\
\hline 38 & & Phomopsichin D & Phomopsis sp. 33\# & R. stylosa (M) & $\begin{array}{l}\text { Antimicrobial, } \\
\text { Antioxidant, } \\
\text { Inhibiting } \\
\text { acetylcholinesterase } \\
\text { and } \alpha \text {-glucosidase }\end{array}$ & [33] \\
\hline 39 & & Chaetocyclinone B & $\begin{array}{l}\text { Phomopsis sp. } \\
\text { HNY29-2B }\end{array}$ & A. ilicifolius (M) & Cytotoxic & [36] \\
\hline 40 & & Pestalotiopsone F & $\begin{array}{l}\text { Phomopsis sp. } \\
\text { IFB-ZS1-S4 }\end{array}$ & $\begin{array}{c}\text { Scaevola } \\
\text { hainanensis (M) }\end{array}$ & $\begin{array}{c}\text { Inhibiting } \\
\text { neuraminidase }\end{array}$ & [37] \\
\hline 41 & & Phomoxanthone F & $\begin{array}{c}\text { Phomopsis sp. } \\
\text { xy21 }\end{array}$ & $\begin{array}{l}\text { Xylocarpus } \\
\text { granatum }(\mathrm{M})\end{array}$ & Anti-HIV & [38] \\
\hline 42 & & $\begin{array}{l}\text { 5-Hydroxy-3-hydroxymethyl- } \\
\text { 2-methyl-7- } \\
\text { methoxychromone }\end{array}$ & $\begin{array}{l}\text { Phomopsis sp. (No. } \\
\text { Gx-4) }\end{array}$ & Sediment (M) & $\begin{array}{l}\text { Cytotoxic, Inhibiting } \\
\text { the growth of SIV } \\
\text { branch }\end{array}$ & [39] \\
\hline 43 & & Phomochromone A & Phomopsis sp. & $\begin{array}{c}\text { Cistus } \\
\text { monspeliensis }(\mathrm{T})\end{array}$ & $\begin{array}{c}\text { Antimicrobial, } \\
\text { Antialgal }\end{array}$ & [40] \\
\hline 44 & & Phomochromone B & Phomopsis sp. & $\begin{array}{l}\text { C. monspeliensis } \\
(\mathrm{T})\end{array}$ & $\begin{array}{c}\text { Antimicrobial, } \\
\text { Antialgal }\end{array}$ & [40] \\
\hline 45 & & Phomochromanone A & $\begin{array}{c}\text { Phomopsis sp. } \\
\text { CGMCC No. } 5416\end{array}$ & $\begin{array}{l}\text { Achyranthes } \\
\text { bidentata }(\mathrm{T})\end{array}$ & Cytotoxic, Anti-HIV & [41] \\
\hline 46 & & Phomochromanone B & $\begin{array}{c}\text { Phomopsis sp. } \\
\text { CGMCC No. } 5416\end{array}$ & A. bidentata $(\mathrm{T})$ & Cytotoxic, Anti-HIV & [41] \\
\hline 47 & & $\begin{array}{l}\text { 5-Hydroxy-6,8-dimethoxy-2- } \\
\text { benzyl-4H-naphtho[2,3-b]- } \\
\text { pyran-4-one }\end{array}$ & $\begin{array}{l}\text { Phomopsis sp. } \\
\text { ZSU-H26 }\end{array}$ & E. agallocha (M) & Cytotoxic & [42] \\
\hline 48 & & Phomopsis-H76 A & $\begin{array}{l}\text { Phomopsis sp. } \\
\text { (\#zsu-H76) }\end{array}$ & E. agallocha (M) & $\begin{array}{l}\text { Accelerating the } \\
\text { growth of SIV branch }\end{array}$ & [43] \\
\hline 49 & Chromanones & $\begin{array}{c}\text { (3R,4S)-3,4-Dihydro-4,5,8- } \\
\text { trihydroxy-3- } \\
\text { methylisocoumarin }\end{array}$ & $\begin{array}{c}\text { Phomopsis sp. (No. } \\
\text { ZH-111) }\end{array}$ & Sediment (M) & $\begin{array}{l}\text { Accelerating the } \\
\text { growth of SIV branch, } \\
\text { Cytotoxic }\end{array}$ & [44] \\
\hline 50 & & $\begin{array}{c}\text { (3R,4S)-3,4-Dihydro-8- } \\
\text { hydroxy-4-methoxy-3- } \\
\text { methylisocoumarin }\end{array}$ & $\begin{array}{l}\text { Phomopsis sp. (No. } \\
\text { Gx-4) }\end{array}$ & Sediment (M) & $\begin{array}{l}\text { Cytotoxic, } \\
\text { Accelerating the } \\
\text { growth of SIV branch }\end{array}$ & [39] \\
\hline 51 & & $\begin{array}{l}\text { 3,4-Dihydro-8-hydroxy-3- } \\
\text { methyl-1H-2-benzopyran-1- } \\
\text { one-5-carboxylic } \\
\text { acid }\end{array}$ & $\begin{array}{l}\text { Phomopsis sp. (No. } \\
\text { Gx-4) }\end{array}$ & Sediment (M) & $\begin{array}{l}\text { Cytotoxic, } \\
\text { Accelerating the } \\
\text { growth of SIV branch }\end{array}$ & [39] \\
\hline 52 & & $\begin{array}{l}\text { 5,8-Dihydroxy-4- } \\
\text { methylcoumarin }\end{array}$ & $\begin{array}{l}\text { Phomopsis sp. (No. } \\
\text { Gx-4) }\end{array}$ & Sediment (M) & $\begin{array}{l}\text { Cytotoxic, Inhibiting } \\
\text { the growth of SIV } \\
\text { branch }\end{array}$ & [39] \\
\hline 53 & & (10S)-Diaporthin & $\begin{array}{c}\text { Phomopsis sp. } \\
\text { sh917 }\end{array}$ & $\begin{array}{l}\text { Isodon eriocalyx } \\
\text { var. laxiflora }(\mathrm{T})\end{array}$ & Antiangiogenic & [45] \\
\hline 54 & & Cytosporone D & $\begin{array}{l}\text { Phomopsis sp. } \\
\text { CMU-LMA }\end{array}$ & $\begin{array}{l}\text { Alpinia malacensis } \\
\text { (T) }\end{array}$ & $\begin{array}{l}\text { Antimicrobial, } \\
\text { Inibiting DnaG } \\
\text { primase }\end{array}$ & [46] \\
\hline 55 & & Alternariol & $\begin{array}{l}\text { Phomopsis sp. } \\
\text { A240 } \\
\text { Phomopsis sp. } \\
\text { CAFT69 } \\
\text { Phomopsis sp. }\end{array}$ & $\begin{array}{c}\text { Taxus chinensis } \\
\text { var. mairei }(\mathrm{T}) \\
\text { Endodesmia } \\
\text { calophylloides }(\mathrm{T}) \\
\text { Senna spectabilis } \\
\text { (T) }\end{array}$ & $\begin{array}{l}\text { Cytotoxic } \\
\text { Motility inhibitory } \\
\text { and zoosporicidal } \\
\text { potential } \\
\text { Anti-inflammatory }\end{array}$ & $\begin{array}{l}{[47]} \\
{[48]} \\
{[49]}\end{array}$ \\
\hline 56 & & Alternariol-5-O-methyl ether & $\begin{array}{l}\text { Phomopsis sp. } \\
\text { CAFT69 }\end{array}$ & $\begin{array}{l}\text { E. calophylloides } \\
\text { (T) }\end{array}$ & $\begin{array}{l}\text { Motility inhibitory } \\
\text { and zoosporicidal } \\
\text { potential }\end{array}$ & [48] \\
\hline
\end{tabular}


Table 1. Cont.

\begin{tabular}{|c|c|c|c|c|c|c|}
\hline Number & Structural Types & Compounds & Strains & $\begin{array}{l}\text { Habitats } \\
\left(\mathrm{T} / \mathrm{M}^{\mathrm{a}}\right)\end{array}$ & Activities & Refs. \\
\hline 57 & & 5'-Hydroxyalternariol & $\begin{array}{l}\text { Phomopsis sp. } \\
\text { A240 } \\
\text { Phomopsis sp. } \\
\text { CAFT69 }\end{array}$ & $\begin{array}{l}\text { T. chinensis var. } \\
\text { mairei }(\mathrm{T}) \\
\text { E. calophylloides } \\
\quad(\mathrm{T})\end{array}$ & $\begin{array}{c}\text { Antioxidant } \\
\text { Motility inhibitory } \\
\text { and zoosporicidal } \\
\text { potential }\end{array}$ & $\begin{array}{l}{[47]} \\
{[48]}\end{array}$ \\
\hline 58 & & Phomochromanone C & $\begin{array}{c}\text { Phomopsis sp. } \\
\text { CGMCC No. } 5416\end{array}$ & A. bidentata $(\mathrm{T})$ & $\begin{array}{c}\text { Cytotoxic, } \\
\text { Pro-apoptotic }\end{array}$ & [41] \\
\hline 59 & Benzofuranones & $\begin{array}{c}\text { 7-Methoxy-6-methyl-3-oxo- } \\
\text { 1,3-dihydroisobenzofuran-4- } \\
\text { carboxylic } \\
\text { acid }\end{array}$ & $\begin{array}{l}\text { Phomopsis sp. } \\
\text { A123 }\end{array}$ & $\begin{array}{l}\text { Kandelia candel } \\
\text { (M) }\end{array}$ & $\begin{array}{l}\text { Cytotoxic, Antifungal, } \\
\text { Antioxidant }\end{array}$ & [50] \\
\hline 60 & & Diaporthelactone & $\begin{array}{l}\text { Phomopsis sp. } \\
\text { A123 }\end{array}$ & K. candel (M) & $\begin{array}{l}\text { Cytotoxic, Antifungal, } \\
\text { Antioxidant }\end{array}$ & [50] \\
\hline 61 & & $\begin{array}{l}\text { 7-Hydroxy-4,6-dimethy-3H- } \\
\text { isobenzofuran-1-one }\end{array}$ & $\begin{array}{l}\text { Phomopsis sp. } \\
\text { A123 }\end{array}$ & K. candel (M) & $\begin{array}{l}\text { Cytotoxic, Antifungal, } \\
\text { Antioxidant }\end{array}$ & [50] \\
\hline 62 & & $\begin{array}{l}\text { 7-Methoxy-4,6-dimethyl-3H- } \\
\text { isobenzofuran-1-one }\end{array}$ & $\begin{array}{l}\text { Phomopsis sp. } \\
\text { A123 }\end{array}$ & K. candel (M) & $\begin{array}{l}\text { Cytotoxic, Antifungal, } \\
\text { Antioxidant }\end{array}$ & [50] \\
\hline 63 & & $\begin{array}{l}\text { 4-(Hydroxymethyl)-7- } \\
\text { methoxy-6-methyl-1(3H)- } \\
\text { isobenzofuranone }\end{array}$ & $\begin{array}{l}\text { Phomopsis sp. (No. } \\
\text { ZH-111) }\end{array}$ & Sediment (M) & $\begin{array}{l}\text { Inhibiting the growth } \\
\text { of SIV branch, } \\
\text { Cytotoxic }\end{array}$ & [44] \\
\hline 64 & & Cytosporone E & $\begin{array}{l}\text { Phomopsis sp. } \\
\text { BCC } 45011\end{array}$ & X. granatum $(\mathrm{M})$ & $\begin{array}{c}\text { Cytotoxic, } \\
\text { Antimalarial }\end{array}$ & [51] \\
\hline 65 & & Cytosporone $\mathrm{P}$ & $\begin{array}{l}\text { Phomopsis sp. } \\
\text { BCC } 45011\end{array}$ & X. granatum $(\mathrm{M})$ & Antimalarial & [51] \\
\hline 66 & & Phomopsidone A & $\begin{array}{l}\text { Phomopsis sp. } \\
\text { A123 }\end{array}$ & K. candel (M) & $\begin{array}{l}\text { Cytotoxic, Antifungal, } \\
\text { Antioxidant }\end{array}$ & [50] \\
\hline 67 & & Excelsione & $\begin{array}{l}\text { Phomopsis sp. } \\
\text { A123 }\end{array}$ & K. candel (M) & $\begin{array}{l}\text { Cytotoxic, Antifungal, } \\
\text { Antioxidant }\end{array}$ & [50] \\
\hline 68 & & Excelsional & $\begin{array}{l}\text { Phomopsis sp. } \\
\text { CAFT69 }\end{array}$ & $\begin{array}{l}\text { E. calophylloides } \\
\text { (T) }\end{array}$ & $\begin{array}{l}\text { Motility inhibitory } \\
\text { and zoosporicidal } \\
\text { potential }\end{array}$ & [48] \\
\hline 69 & & Lithocarol A & $\begin{array}{l}\text { P. lithocarpus } \\
\text { FS508 }\end{array}$ & Sediment (M) & Cytotoxic & [52] \\
\hline 70 & & Lithocarol B & $\begin{array}{l}\text { P. lithocarpus } \\
\text { FS508 }\end{array}$ & Sediment (M) & Cytotoxic & [52] \\
\hline 71 & & Lithocarol C & $\begin{array}{l}\text { P. lithocarpus } \\
\text { FS508 }\end{array}$ & Sediment (M) & Cytotoxic & [52] \\
\hline 72 & & Lithocarol D & $\begin{array}{l}\text { P. lithocarpus } \\
\text { FS508 }\end{array}$ & Sediment (M) & Cytotoxic & [52] \\
\hline 73 & & Lithocarol E & $\begin{array}{l}\text { P. lithocarpus } \\
\text { FS508 }\end{array}$ & Sediment (M) & Cytotoxic & [52] \\
\hline 74 & & Lithocarol F & $\begin{array}{l}\text { P. lithocarpus } \\
\text { FS508 }\end{array}$ & Sediment (M) & Cytotoxic & [52] \\
\hline 75 & & Isoprenylisobenzofuran A & $\begin{array}{l}\text { P. lithocarpus } \\
\text { FS508 }\end{array}$ & Sediment (M) & Cytotoxic & [52] \\
\hline 76 & & $\begin{array}{l}\text { 7-Methoxy-2-(4- } \\
\text { methoxyphenyl)-3-methyl-5- } \\
\text { (3-prenyl)-benzofuran }\end{array}$ & Phomopsis sp. & $\begin{array}{l}\text { P. polyphylla var. } \\
\text { yunnanensis }(\mathrm{T})\end{array}$ & Anti-TMV & [53] \\
\hline 77 & & $\begin{array}{l}\text { 2-(4-Methoxyphenyl)-3- } \\
\text { methyl-5-(3-prenyl)- } \\
\text { benzofuran-7-ol }\end{array}$ & Phomopsis sp. & $\begin{array}{l}\text { P. polyphylla var. } \\
\text { yunnanensis }(\mathrm{T})\end{array}$ & Anti-TMV & [53] \\
\hline 78 & & $\begin{array}{c}\text { 2-(4-Hydroxy-3,5- } \\
\text { dimethoxyphenyl)-3-methyl- } \\
\text { 5-(3-prenyl) } \\
\text { benzofuran-7-ol }\end{array}$ & Phomopsis sp. & $\begin{array}{l}\text { P. polyphylla var. } \\
\text { yunnanensis }(\mathrm{T})\end{array}$ & Anti-TMV & [53] \\
\hline 79 & & Moracin N & Phomopsis sp. & $\begin{array}{l}\text { P. polyphylla var. } \\
\text { yunnanensis }(\mathrm{T})\end{array}$ & Anti-TMV & [53] \\
\hline 80 & & $\begin{array}{l}\text { 2-(2'-Methoxy-4'-hydroxy)- } \\
\text { aryl-3-methy-6- } \\
\text { hydroxybenzofuran }\end{array}$ & Phomopsis sp. & $\begin{array}{l}\text { P. polyphylla var. } \\
\text { yunnanensis }(\mathrm{T})\end{array}$ & Anti-TMV & [53] \\
\hline 81 & & Iteafuranal B & Phomopsis sp. & $\begin{array}{l}\text { P. polyphylla var. } \\
\text { yunnanensis }(\mathrm{T})\end{array}$ & Anti-TMV & [53] \\
\hline 82 & & Moracin P & Phomopsis sp. & $\begin{array}{l}\text { P. polyphylla var. } \\
\text { yunnanensis }(\mathrm{T})\end{array}$ & Anti-TMV & [53] \\
\hline
\end{tabular}


Table 1. Cont.

\begin{tabular}{|c|c|c|c|c|c|c|}
\hline Number & Structural Types & Compounds & Strains & $\begin{array}{l}\text { Habitats } \\
\left(\mathrm{T} / \mathrm{M}^{\mathrm{a}}\right)\end{array}$ & Activities & Refs. \\
\hline 83 & Pyrones & Phomaspyrone A & $\begin{array}{c}\text { P. asparagi } \\
\text { SWUKJ5.2020 }\end{array}$ & $\begin{array}{c}\text { Kadsura } \\
\text { angustifolia }(\mathrm{T})\end{array}$ & Cytotoxic & [54] \\
\hline 84 & & Macommelin-8,9-diol & $\begin{array}{c}\text { P. asparagi } \\
\text { SWUKJ5.2020 }\end{array}$ & K. angustifolia $(\mathrm{T})$ & Cytotoxic & [54] \\
\hline 85 & & Phomaspyrone B & $\begin{array}{c}\text { P. asparagi } \\
\text { SWUKJ5.2020 }\end{array}$ & K. angustifolia $(\mathrm{T})$ & Cytotoxic & [54] \\
\hline 86 & & Phomaspyrone C & $\begin{array}{c}\text { P. asparagi } \\
\text { SWUKJ5.2020 }\end{array}$ & K. angustifolia $(\mathrm{T})$ & Cytotoxic & [54] \\
\hline 87 & & Phomaspyrone D & $\begin{array}{c}\text { P.asparagi } \\
\text { SWUKJ5.2020 }\end{array}$ & K. angustifolia $(\mathrm{T})$ & Cytotoxic & [54] \\
\hline 88 & & Phomaspyrone E & $\begin{array}{c}\text { P. asparagi } \\
\text { SWUKJ5.2020 }\end{array}$ & K. angustifolia $(\mathrm{T})$ & Cytotoxic & [54] \\
\hline 89 & & Macommelin-9-ol & $\begin{array}{c}\text { P.asparagi } \\
\text { SWUKJ5.2020 }\end{array}$ & K. angustifolia $(\mathrm{T})$ & Cytotoxic & [54] \\
\hline 90 & & Macommelin & $\begin{array}{c}\text { P. asparagi } \\
\text { SWUKJ5.2020 }\end{array}$ & K. angustifolia (T) & Cytotoxic & [54] \\
\hline 91 & & Pyrenocine J & Phomopsis sp. & $\begin{array}{l}\text { Cistus salvifolius } \\
\text { (T) }\end{array}$ & $\begin{array}{l}\text { Antifungal, } \\
\text { Antibacterial, } \\
\text { Algicidal }\end{array}$ & [55] \\
\hline 92 & & Pyrenocine $\mathrm{K}$ & Phomopsis sp. & C. salvifolius $(\mathrm{T})$ & $\begin{array}{l}\text { Antifungal, } \\
\text { Antibacterial, } \\
\text { Algicidal }\end{array}$ & [55] \\
\hline 93 & & Pyrenocine L & Phomopsis sp. & C. salvifolius $(\mathrm{T})$ & $\begin{array}{l}\text { Antibacterial, } \\
\text { Algicidal }\end{array}$ & [55] \\
\hline 94 & & Pyrenocine $\mathrm{M}$ & Phomopsis sp. & C. salvifolius $(\mathrm{T})$ & $\begin{array}{c}\text { Antifungal, } \\
\text { Antibacterial, } \\
\text { Algicidal }\end{array}$ & [55] \\
\hline 95 & & Phomopsis-H76 C & $\begin{array}{l}\text { Phomopsis sp. } \\
\text { (\#zsu-H76) }\end{array}$ & E. agallocha $(\mathrm{M})$ & $\begin{array}{l}\text { Inhibiting the growth } \\
\text { of SIV branch }\end{array}$ & [43] \\
\hline 96 & Quinones & Anhydrojavanicin & $\begin{array}{l}\text { Phomopsis sp. } \\
\text { HCCB04730 }\end{array}$ & $\begin{array}{l}\text { Radix Stephaniae } \\
\text { Japonicae }(\mathrm{T})\end{array}$ & Cytotoxic, Anti-HIV & [56] \\
\hline 97 & & Dihydroanhydrojavanicin & $\begin{array}{l}\text { Phomopsis sp. } \\
\text { HCCB04730 }\end{array}$ & $\begin{array}{l}\text { Radix Stephaniae } \\
\text { Japonicae }(\mathrm{T})\end{array}$ & Cytotoxic, Anti-HIV & [56] \\
\hline 98 & & Fusarubin & $\begin{array}{l}\text { Phomopsis sp. } \\
\text { HCCB04730 }\end{array}$ & $\begin{array}{l}\text { Radix Stephaniae } \\
\text { Japonicae }(\mathrm{T})\end{array}$ & Cytotoxic, Anti-HIV & [56] \\
\hline 99 & & Javanicin & $\begin{array}{l}\text { Phomopsis sp. } \\
\text { HCCB04730 }\end{array}$ & $\begin{array}{l}\text { Radix Stephaniae } \\
\text { Japonicae }(\mathrm{T})\end{array}$ & Cytotoxic, Anti-HIV & [56] \\
\hline 100 & & $\begin{array}{l}\text { 2-Acetonyl-3methyl-5- } \\
\text { hydroxy-7-methoxy- } \\
\text { naphthazarin }\end{array}$ & $\begin{array}{l}\text { Phomopsis sp. } \\
\text { HCCB04730 }\end{array}$ & $\begin{array}{l}\text { Radix Stephaniae } \\
\text { Japonicae }(\mathrm{T})\end{array}$ & Cytotoxic, Anti-HIV & [56] \\
\hline 101 & & Bostrycoidin & $\begin{array}{l}\text { Phomopsis sp. } \\
\text { HCCB04730 }\end{array}$ & $\begin{array}{l}\text { Radix Stephaniae } \\
\text { Japonicae }(\mathrm{T})\end{array}$ & Cytotoxic, Anti-HIV & [56] \\
\hline 102 & & Altersolanol B & $\begin{array}{l}\text { P. longicolla } \\
\text { HL-2232 }\end{array}$ & $\begin{array}{l}\text { Bruguiera } \\
\text { sexangula var. } \\
\text { rhynchopetala }(\mathrm{M})\end{array}$ & Antibacterial & [57] \\
\hline 103 & & Altersolanol A & $\begin{array}{l}\text { Phomopsis sp. } \\
\text { (PM0409092) } \\
\text { P. foeniculi }\end{array}$ & $\begin{array}{l}\text { Nyctanthes } \\
\text { arbor-tristis }(\mathrm{T}) \\
\text { Foeniculum } \\
\text { vulgare }(\mathrm{T})\end{array}$ & $\begin{array}{l}\text { Cytotoxic } \\
\text { Phytotoxic }\end{array}$ & $\begin{array}{l}{[58]} \\
{[59]}\end{array}$ \\
\hline 104 & & $\begin{array}{l}(2 R, 3 S)-7-\text {-Ethyl-1,2,3,4- } \\
\text { tetrahydro-2,3,8-trihdroxy-6- } \\
\text { methoxy-3-methyl-9,10- } \\
\text { anthracenedione }\end{array}$ & $\begin{array}{l}\text { Phomopsis sp. } \\
\text { PSU-MA214 }\end{array}$ & $\begin{array}{l}\text { Rhizophora } \\
\text { apiculata }(\mathrm{M})\end{array}$ & $\begin{array}{l}\text { Cytotoxic, } \\
\text { Antibacterial }\end{array}$ & [60] \\
\hline 105 & & Altersolanol J & P. foeniculi & F. vulgare $(\mathrm{T})$ & Phytotoxic & [59] \\
\hline 106 & & $\begin{array}{l}\text { 2-Hydroxymethyl- } 4 \beta, 5 \alpha, 6 \beta \text { - } \\
\text { trihydroxycyclohex-2-en }\end{array}$ & Phomopsis sp. & $\begin{array}{l}\text { Notobasis syriaca } \\
\text { (T) }\end{array}$ & $\begin{array}{l}\text { Antibacterial, } \\
\text { Algicidal } \\
\text { Antifungal, }\end{array}$ & [61] \\
\hline 107 & & (-)-Phyllostine & Phomopsis sp. & N. syriaca $(\mathrm{T})$ & $\begin{array}{l}\text { Antibacterial, } \\
\text { Algicidal }\end{array}$ & [61] \\
\hline 108 & & (+)-Epiepoxydon & Phomopsis sp. & N. syriaca $(\mathrm{T})$ & $\begin{array}{l}\text { Antibacterial, } \\
\text { Algicidal }\end{array}$ & [61] \\
\hline 109 & & $(+)$-Epoxydon monoacetate & Phomopsis sp. & N. syriaca $(\mathrm{T})$ & $\begin{array}{l}\text { Antifungal, } \\
\text { Antibacterial, } \\
\text { Algicidal }\end{array}$ & [61] \\
\hline 110 & & Phomonaphthalenone A & $\begin{array}{l}\text { Phomopsis sp. } \\
\text { HCCB04730 }\end{array}$ & $\begin{array}{l}\text { Radix Stephaniae } \\
\text { Japonicae }(\mathrm{T})\end{array}$ & Cytotoxic, Anti-HIV & [56] \\
\hline
\end{tabular}


Table 1. Cont.

\begin{tabular}{|c|c|c|c|c|c|c|}
\hline Number & Structural Types & Compounds & Strains & $\begin{array}{l}\text { Habitats } \\
\left(\mathrm{T} / \mathbf{M}^{\mathrm{a}}\right)\end{array}$ & Activities & Refs. \\
\hline 111 & & Ampelanol & $\begin{array}{c}\text { Phomopsis sp. } \\
\text { HNY29-2B }\end{array}$ & A. ilicifolius (M) & Antibacterial & [62] \\
\hline 112 & Phenols & Phomosine K & Phomopsis sp. & N. syriaca $(\mathrm{T})$ & Antibacterial & [61] \\
\hline 113 & & Phomosine A & Phomopsis sp. & $\begin{array}{l}\text { Ligustrum vulgare } \\
(\mathrm{T})\end{array}$ & $\begin{array}{l}\text { Antifungal, } \\
\text { Antibacterial, } \\
\text { Inhibiting algae }\end{array}$ & [63] \\
\hline 114 & & Phomosine B & Phomopsis sp. & L. vulgare $(\mathrm{T})$ & $\begin{array}{l}\text { Antifungal, } \\
\text { Antibacterial }\end{array}$ & [63] \\
\hline 115 & & Phomosine C & Phomopsis sp. & L. vulgare $(\mathrm{T})$ & $\begin{array}{c}\text { Antifungal, } \\
\text { Antibacterial }\end{array}$ & [63] \\
\hline 116 & & Phomosine D & Phomopsis sp. & L. vulgare $(\mathrm{T})$ & $\begin{array}{l}\text { Antifungal, Inhibiting } \\
\text { algae }\end{array}$ & [63] \\
\hline 117 & & Phomosine I & Phomopsis sp. & L. vulgare $(\mathrm{T})$ & $\begin{array}{c}\text { Antifungal, } \\
\text { Antibacterial }\end{array}$ & [63] \\
\hline 118 & & $\begin{array}{l}\text { 4-(3-Methoxy-5- } \\
\text { methylphenoxy)-2-(2- } \\
\text { hydroxyethyl)-6- } \\
\text { (hydroxymethyl)phenol }\end{array}$ & P. asparagi & $\begin{array}{l}\text { P. polyphylla var. } \\
\text { yunnanensis }(\mathrm{T})\end{array}$ & Anti-MRSA & {$[64]$} \\
\hline 119 & & $\begin{array}{c}\text { 4-(3-Hydroxy-5- } \\
\text { methylphenoxy)-2-(2- } \\
\text { hydroxyethyl)-6- } \\
\text { (hydroxymethyl)phenol }\end{array}$ & P. asparagi & $\begin{array}{l}\text { P. polyphylla var. } \\
\text { yunnanensis }(\mathrm{T})\end{array}$ & Anti-MRSA & {$[64]$} \\
\hline 120 & & $\begin{array}{l}\text { 4-(3-Methoxy-5- } \\
\text { methylphenoxy)-2-(2- } \\
\text { hydroxyethyl)-6- } \\
\text { methylphenol }\end{array}$ & P. fukushii & $\begin{array}{l}\text { P. polyphylla var. } \\
\text { yunnanensis }(\mathrm{T})\end{array}$ & Anti-MRSA & [65] \\
\hline 121 & & $\begin{array}{l}\text { 4-(3-Hydroxy-5- } \\
\text { methylphenoxy)-2-(2- } \\
\text { hydroxyethyl)-6- } \\
\text { methylphenol }\end{array}$ & P. fukushii & $\begin{array}{l}\text { P. polyphylla var. } \\
\text { yunnanensis }(\mathrm{T})\end{array}$ & Anti-MRSA & {$[65]$} \\
\hline 122 & & $\begin{array}{c}\text { 4-(3-Methoxy-5- } \\
\text { methylphenoxy)-2-(3- } \\
\text { hydroxypropyl)-6- } \\
\text { methylphenol }\end{array}$ & P. fukushii & $\begin{array}{l}\text { P. polyphylla var. } \\
\text { yunnanensis }(\mathrm{T})\end{array}$ & Anti-MRSA & [65] \\
\hline 123 & & $\begin{array}{l}\text { 1-(4-(3-Methoxy-5- } \\
\text { methylphenoxy)-2-methoxy- } \\
\text { 6-methylphenyl)-3- } \\
\text { methylbut-3-en-2-one }\end{array}$ & P. fukushii & $\begin{array}{l}\text { P. polyphylla var. } \\
\text { yunnanensis }(\mathrm{T})\end{array}$ & Anti-MRSA & [66] \\
\hline 124 & & $\begin{array}{l}\text { 1-(4-(3-(Hydroxymethyl)- } \\
\text { 5methoxyphenoxy)-2- } \\
\text { methoxy-6-methylphenyl)-3- } \\
\text { methylbut-3-en-2-one }\end{array}$ & P. fukushii & $\begin{array}{l}\text { P. polyphylla var. } \\
\text { yunnanensis }(\mathrm{T})\end{array}$ & Anti-MRSA & [66] \\
\hline 125 & & $\begin{array}{c}\text { 1-(4-(3-Hydroxy- } \\
\text { 5(hydroxymethyl)phenoxy)-2- } \\
\text { methoxy-6-methylphenyl)-3- } \\
\text { methylbut-3-en-2-one }\end{array}$ & P. fukushii & $\begin{array}{l}\text { P. polyphylla var. } \\
\text { yunnanensis }(\mathrm{T})\end{array}$ & Anti-MRSA & [66] \\
\hline 126 & & $\begin{array}{c}\text { 1-[2-Methoxy-4-(3-methoxy-5- } \\
\text { methylphenoxy)-6- } \\
\text { methylphenyl]-ethanone }\end{array}$ & P. fukushii & $\begin{array}{l}\text { P. polyphylla var. } \\
\text { yunnanensis }(\mathrm{T})\end{array}$ & Anti-MRSA & [67] \\
\hline 127 & & $\begin{array}{l}\text { 1-[4-(3-(Hydroxymethyl)-5- } \\
\text { methoxyphenoxy)-2- } \\
\text { methoxy-6-methylphenyl]- } \\
\text { ethanone }\end{array}$ & P. fukushii & $\begin{array}{l}\text { P. polyphylla var. } \\
\text { yunnanensis }(\mathrm{T})\end{array}$ & Anti-MRSA & [67] \\
\hline 128 & & $\begin{array}{l}\text { 3-Hydroxy-1-(1,8-dihydroxy- } \\
\text { 3,6-dimethoxynaphthalen-2- } \\
\text { yl)propan-1-one }\end{array}$ & P. fukushii & $\begin{array}{l}\text { P. polyphylla var. } \\
\text { yunnanensis }(\mathrm{T})\end{array}$ & Anti-MRSA & [68] \\
\hline 129 & & $\begin{array}{l}\text { 3-Hydroxy-1-(1,3,8- } \\
\text { trihydroxy-6- } \\
\text { methoxynaphthalen-2- } \\
\text { yl)propan-1-one }\end{array}$ & P. fukushii & $\begin{array}{l}\text { P. polyphylla var. } \\
\text { yunnanensis }(\mathrm{T})\end{array}$ & Anti-MRSA & [68] \\
\hline 130 & & $\begin{array}{l}\text { 3-Hydroxy-1-(1,8-dihydroxy- } \\
\text { 3,5-dimethoxynaphthalen-2- } \\
\text { yl)propan-1-one }\end{array}$ & P. fukushii & $\begin{array}{l}\text { P. polyphylla var. } \\
\text { yunnanensis }(\mathrm{T})\end{array}$ & Anti-MRSA & [68] \\
\hline 131 & & $\begin{array}{l}\text { 5-Methoxy-2-methyl-7-(3- } \\
\text { methyl-2-oxobut-3-enyl)-1- } \\
\text { naphthaldehyde }\end{array}$ & Phomopsis sp. & $\begin{array}{l}\text { P. polyphylla var. } \\
\text { yunnanensis }(\mathrm{T})\end{array}$ & Anti-MRSA & [69] \\
\hline
\end{tabular}


Table 1. Cont.

\begin{tabular}{|c|c|c|c|c|c|c|}
\hline Number & Structural Types & Compounds & Strains & $\begin{array}{l}\text { Habitats } \\
\left(\mathrm{T} / \mathrm{M}^{\mathrm{a}}\right)\end{array}$ & Activities & Refs. \\
\hline 132 & & $\begin{array}{l}\text { 2-(Hydroxymethyl)-5- } \\
\text { methoxy-7-(3-methyl-2- } \\
\text { oxobut-3-enyl)-1- } \\
\text { naphthaldehyde }\end{array}$ & Phomopsis sp. & $\begin{array}{l}\text { P. polyphylla var. } \\
\text { yunnanensis }(\mathrm{T})\end{array}$ & Anti-MRSA & [69] \\
\hline 133 & & Tenellone $\mathrm{H}$ & $\begin{array}{l}\text { P. lithocarpus } \\
\text { FS508 }\end{array}$ & Sediment (M) & Cytotoxic & [70] \\
\hline 134 & & 16-Acetoxycytosporone B & $\begin{array}{c}\text { Phomopsis sp. YM } \\
355364\end{array}$ & A. carmichaeli $(\mathrm{T})$ & Antifungal & [71] \\
\hline 135 & & Cytosporone B & $\begin{array}{c}\text { Phomopsis sp. } \\
0391 \\
\text { Phomopsis sp. } \\
\text { PSU-H188 }\end{array}$ & $\begin{array}{c}\text { P. polyphylla var. } \\
\text { yunnanensis }(\mathrm{T}) \\
\text { Hevea brasiliensis } \\
\text { (T) }\end{array}$ & $\begin{array}{c}\text { Inhibiting lipase } \\
\text { Protecting pancreatic } \\
\beta \text {-cells }\end{array}$ & $\begin{array}{l}{[72]} \\
{[73]}\end{array}$ \\
\hline 136 & & Dothiorelone A & $\begin{array}{c}\text { Phomopsis sp. } \\
0391\end{array}$ & $\begin{array}{l}\text { P. polyphylla var. } \\
\text { yunnanensis }(\mathrm{T})\end{array}$ & Inhibiting lipase & [72] \\
\hline 137 & & Lithocarpinol A & $\begin{array}{l}\text { P. lithocarpus } \\
\text { FS508 }\end{array}$ & Sediment (M) & Cytotoxic & [74] \\
\hline 138 & & Lithocarpinol B & $\begin{array}{c}\text { P. lithocarpus } \\
\text { FS508 }\end{array}$ & Sediment (M) & Cytotoxic & [74] \\
\hline 139 & & Phomoindene A & $\begin{array}{l}\text { Phomopsis sp. (No. } \\
\text { GX7-4A) }\end{array}$ & Sediment (M) & Cytotoxic & [75] \\
\hline 140 & & 4-Hydroxybenzaldehyde & $\begin{array}{c}\text { Phomopsis sp. YM } \\
355364\end{array}$ & A. carmichaelii $(\mathrm{T})$ & Antimicrobial & [26] \\
\hline 141 & & $\begin{array}{c}5,5^{\prime} \text {-Dimethoxybiphenyl-2,2' }- \\
\text { diol }\end{array}$ & $\begin{array}{l}\text { P. longicolla } \\
\text { HL-2232 }\end{array}$ & $\begin{array}{l}\text { B. sexangula var. } \\
\text { rhynchopetala }(\mathrm{M})\end{array}$ & Antibacterial & [57] \\
\hline 142 & & Phomonitroester & $\begin{array}{l}\text { Phomopsis sp. } \\
\text { PSU-MA214 }\end{array}$ & R. apiculate (M) & Cytotoxic & [60] \\
\hline 143 & & Cytosporone $\mathrm{U}$ & $\begin{array}{l}\text { Phomopsis sp. } \\
\text { FJBR-11 }\end{array}$ & Brucea javanica $(\mathrm{T})$ & Anti-TMV & [76] \\
\hline 144 & & Altenusin & $\begin{array}{l}\text { Phomopsis sp. } \\
\text { CAFT69 }\end{array}$ & $\begin{array}{l}\text { E. calophylloides } \\
\text { (T) }\end{array}$ & $\begin{array}{l}\text { Motility inhibitory } \\
\text { and zoosporicidal } \\
\text { potential }\end{array}$ & [48] \\
\hline 145 & & Cosmochlorin D & $\begin{array}{l}\text { Phomopsis sp. } \\
\quad \text { N-125 }\end{array}$ & Ficus ampelas $(\mathrm{T})$ & $\begin{array}{c}\text { Cytotoxic, } \\
\text { Growth-inhibition } \\
\text { activity }\end{array}$ & [77] \\
\hline 146 & & Cosmochlorin E & $\begin{array}{l}\text { Phomopsis sp. } \\
\quad \text { N-125 }\end{array}$ & F. ampelas $(\mathrm{T})$ & $\begin{array}{c}\text { Cytotoxic, } \\
\text { Growth-inhibition } \\
\text { activity }\end{array}$ & [77] \\
\hline 147 & Oblongolides & Oblongolide Z & $\begin{array}{l}\text { Phomopsis sp. } \\
\text { BCC } 9789\end{array}$ & $\begin{array}{l}\text { Musa acuminate } \\
(\mathrm{T})\end{array}$ & $\begin{array}{l}\text { Cytotoxic, } \\
\text { Anti-HSV-1 }\end{array}$ & [78] \\
\hline 148 & & Oblongolide Y & $\begin{array}{l}\text { Phomopsis sp. } \\
\text { BCC } 9789\end{array}$ & M. acuminate (T) & Cytotoxic & [78] \\
\hline 149 & & Oblongolide $\mathrm{C} 1$ & $\begin{array}{l}\text { Phomopsis sp. } \\
\text { XZ-01 }\end{array}$ & $\begin{array}{l}\text { Camptotheca } \\
\text { acuminate }(\mathrm{T})\end{array}$ & Cytotoxic & [79] \\
\hline 150 & & Oblongolide P1 & $\begin{array}{l}\text { Phomopsis sp. } \\
\text { XZ-01 }\end{array}$ & C. acuminate $(\mathrm{T})$ & Cytotoxic & [79] \\
\hline 151 & & Oblongolide X1 & $\begin{array}{l}\text { Phomopsis sp. } \\
\text { XZ-01 }\end{array}$ & C. acuminate $(\mathrm{T})$ & Cytotoxic & [79] \\
\hline 152 & & 6-Hydroxyphomodiol & $\begin{array}{l}\text { Phomopsis sp. } \\
\text { XZ-01 }\end{array}$ & C. acuminate $(\mathrm{T})$ & Cytotoxic & [79] \\
\hline 153 & & Oblongolide C & $\begin{array}{l}\text { Phomopsis sp. } \\
\text { XZ-01 }\end{array}$ & C. acuminate $(\mathrm{T})$ & Cytotoxic & [79] \\
\hline 154 & & $\begin{array}{c}\text { 2-Deoxy- } 4 \alpha- \\
\text { hydroxyoblongolide } \\
X\end{array}$ & $\begin{array}{l}\text { Phomopsis sp. } \\
\text { BCC } 9789\end{array}$ & M. acuminate (T) & Anti-HSV-1 & [78] \\
\hline 155 & $\begin{array}{l}\text { Unclassified } \\
\text { polyketides }\end{array}$ & Phomoxydiene C & $\begin{array}{l}\text { Phomopsis sp. } \\
\text { BCC } 45011\end{array}$ & X. granatum $(\mathrm{M})$ & $\begin{array}{l}\text { Cytotoxic, } \\
\text { Antimalarial }\end{array}$ & [51] \\
\hline 156 & & $1893 \mathrm{~A}$ & $\begin{array}{l}\text { Phomopsis sp. } \\
\text { BCC } 45011\end{array}$ & X. granatum $(\mathrm{M})$ & Cytotoxic & [51] \\
\hline 157 & & Mycoepoxydiene & $\begin{array}{l}\text { Phomopsis sp. } \\
\text { BCC } 45011\end{array}$ & X. granatum $(\mathrm{M})$ & $\begin{array}{l}\text { Cytotoxic, } \\
\text { Antimalarial }\end{array}$ & [51] \\
\hline 158 & & Deacetylmycoepoxydiene & $\begin{array}{l}\text { Phomopsis sp. } \\
\text { BCC } 45011\end{array}$ & $X$. granatum $(\mathrm{M})$ & $\begin{array}{l}\text { Cytotoxic, } \\
\text { Antimalarial }\end{array}$ & [51] \\
\hline 159 & & Phomoxydiene A & $\begin{array}{l}\text { Phomopsis sp. } \\
\text { BCC } 45011\end{array}$ & X. granatum $(\mathrm{M})$ & $\begin{array}{l}\text { Cytotoxic, } \\
\text { Antimalarial }\end{array}$ & [51] \\
\hline
\end{tabular}


Table 1. Cont.

\begin{tabular}{|c|c|c|c|c|c|c|}
\hline Number & Structural Types & Compounds & Strains & $\begin{array}{l}\text { Habitats } \\
\left(T / M^{a}\right)\end{array}$ & Activities & Refs. \\
\hline 160 & & Phomopoxide A & $\begin{array}{l}\text { Phomopsis sp. } \\
\text { YE3250 }\end{array}$ & $\begin{array}{l}\text { Paeonia delavayi } \\
\text { (T) }\end{array}$ & $\begin{array}{c}\text { Cytotoxic, Antifungal, } \\
\text { Inhibiting } \\
\alpha \text {-glycosidase }\end{array}$ & [80] \\
\hline 161 & & Phomopoxide B & $\begin{array}{l}\text { Phomopsis sp. } \\
\text { YE3250 }\end{array}$ & P. delavayi $(\mathrm{T})$ & $\begin{array}{c}\text { Cytotoxic, Antifungal, } \\
\text { Inhibiting } \\
\alpha \text {-glycosidase }\end{array}$ & [80] \\
\hline 162 & & Phomopoxide C & $\begin{array}{l}\text { Phomopsis sp. } \\
\text { YE3250 }\end{array}$ & P.delavayi $(\mathrm{T})$ & $\begin{array}{c}\text { Cytotoxic, Antifungal, } \\
\text { Inhibiting } \\
\alpha \text {-glycosidase }\end{array}$ & [80] \\
\hline 163 & & Phomopoxide D & $\begin{array}{l}\text { Phomopsis sp. } \\
\text { YE3250 }\end{array}$ & P. delavayi $(\mathrm{T})$ & $\begin{array}{c}\text { Cytotoxic, Antifungal, } \\
\text { Inhibiting } \\
\alpha \text {-glycosidase }\end{array}$ & [80] \\
\hline 164 & & Phomopoxide E & $\begin{array}{l}\text { Phomopsis sp. } \\
\text { YE3250 }\end{array}$ & P.delavayi $(\mathrm{T})$ & $\begin{array}{c}\text { Cytotoxic, Antifungal, } \\
\text { Inhibiting } \\
\alpha \text {-glycosidase }\end{array}$ & [80] \\
\hline 165 & & Phomopoxide F & $\begin{array}{l}\text { Phomopsis sp. } \\
\text { YE3250 }\end{array}$ & P. delavayi $(\mathrm{T})$ & $\begin{array}{c}\text { Cytotoxic, Antifungal, } \\
\text { Inhibiting } \\
\alpha \text {-glycosidase }\end{array}$ & [80] \\
\hline 166 & & Phomopoxide G & $\begin{array}{l}\text { Phomopsis sp. } \\
\text { YE3250 }\end{array}$ & P.delavayi $(\mathrm{T})$ & $\begin{array}{c}\text { Cytotoxic, Antifungal, } \\
\text { Inhibiting } \\
\alpha \text {-glycosidase }\end{array}$ & [80] \\
\hline 167 & & Phomentrioloxin & Phomopsis sp. & $\begin{array}{l}\text { Carthamus lanatus } \\
\text { (T) }\end{array}$ & Phytotoxic & [81] \\
\hline 168 & & Phomotenone & Phomopsis sp. & $\begin{array}{c}\text { C. monspeliensis } \\
\text { (T) }\end{array}$ & $\begin{array}{l}\text { Antifungal, } \\
\text { Antibacterial, } \\
\text { Antialgal }\end{array}$ & [40] \\
\hline 169 & & Phomopsolide B & $\begin{array}{l}\text { Phomopsis sp. } \\
\text { DC275 }\end{array}$ & Vitis vinifera $(\mathrm{T})$ & $\begin{array}{l}\text { Antibacterial, } \\
\text { Phytotoxic }\end{array}$ & [82] \\
\hline 170 & & Phomopsolidone A & $\begin{array}{l}\text { Phomopsis sp. } \\
\text { DC275 }\end{array}$ & $V$.vinifera $(\mathrm{T})$ & $\begin{array}{l}\text { Antibacterial, } \\
\text { Phytotoxic }\end{array}$ & [82] \\
\hline 171 & & Phomopsolidone B & $\begin{array}{l}\text { Phomopsis sp. } \\
\text { DC275 }\end{array}$ & $V$. vinifera $(\mathrm{T})$ & $\begin{array}{l}\text { Antibacterial, } \\
\text { Phytotoxic }\end{array}$ & [82] \\
\hline 172 & Monoterpenoids & Acropyrone & $\begin{array}{l}\text { Phomopsis sp. } \\
\text { HNY29-2B }\end{array}$ & A. ilicifolius (M) & Antibacterial & {$[62]$} \\
\hline 173 & & Nectriapyrone & P. foeniculi & F. vulgare $(\mathrm{T})$ & Phytotoxic & [59] \\
\hline 174 & & $\begin{array}{l}(1 S, 2 S, 4 S) \text {-Trihydroxy- } p \text { - } \\
\text { menthane }\end{array}$ & Phomopsis sp. & $\begin{array}{l}\text { C. monspeliensis } \\
\text { (T) }\end{array}$ & $\begin{array}{l}\text { Antibacterial, } \\
\text { Antialgal }\end{array}$ & [40] \\
\hline 175 & Sesquiterpenoids & Phomophyllin A & $\begin{array}{l}\text { Phomopsis sp. } \\
\text { TJ507A }\end{array}$ & $\begin{array}{l}\text { Phyllanthus } \\
\text { glaucus }(\mathrm{T})\end{array}$ & Inhibiting BACE1 & [83] \\
\hline 176 & & Phomophyllin B & $\begin{array}{l}\text { Phomopsis sp. } \\
\text { TJ507A }\end{array}$ & P.glaucus $(\mathrm{T})$ & Inhibiting BACE1 & [83] \\
\hline 177 & & Phomophyllin C & $\begin{array}{l}\text { Phomopsis sp. } \\
\text { TJ507A }\end{array}$ & P.glaucus $(\mathrm{T})$ & Inhibiting BACE1 & [83] \\
\hline 178 & & Phomophyllin D & $\begin{array}{l}\text { Phomopsis sp. } \\
\text { TJ507A }\end{array}$ & P.glaucus $(\mathrm{T})$ & Inhibiting BACE1 & [83] \\
\hline 179 & & Phomophyllin E & $\begin{array}{l}\text { Phomopsis sp. } \\
\text { TJ507A }\end{array}$ & P.glaucus $(\mathrm{T})$ & Inhibiting BACE1 & [83] \\
\hline 180 & & Phomophyllin F & $\begin{array}{l}\text { Phomopsis sp. } \\
\text { TJ507A }\end{array}$ & P.glaucus $(\mathrm{T})$ & Inhibiting BACE1 & [83] \\
\hline 181 & & Phomophyllin G & $\begin{array}{l}\text { Phomopsis sp. } \\
\text { TJ507A }\end{array}$ & P.glaucus $(\mathrm{T})$ & Inhibiting BACE1 & [83] \\
\hline 182 & & Radulone B & $\begin{array}{l}\text { Phomopsis sp. } \\
\text { TJ507A }\end{array}$ & P.glaucus $(\mathrm{T})$ & Inhibiting BACE1 & [83] \\
\hline 183 & & Phomophyllin I & $\begin{array}{l}\text { Phomopsis sp. } \\
\text { TJ507A }\end{array}$ & P.glaucus $(\mathrm{T})$ & Inhibiting BACE1 & [83] \\
\hline 184 & & Onitin & $\begin{array}{l}\text { Phomopsis sp. } \\
\text { TJ507A }\end{array}$ & P.glaucus $(\mathrm{T})$ & Inhibiting BACE1 & [83] \\
\hline 185 & & $\begin{array}{l}(7 R, 9 S, 10 R)-3,9-\mathrm{Di}- \\
\text { hidroxicalamenene }\end{array}$ & P. cassiae & $\begin{array}{l}\text { Cassia spectabilis } \\
\text { (T) }\end{array}$ & $\begin{array}{l}\text { Inhibiting } \\
\text { acetylcholinesterase, } \\
\text { Antifungal }\end{array}$ & [84] \\
\hline 186 & & $\begin{array}{l}(7 R, 9 R, 10 R)-3,9-\mathrm{Di}- \\
\text { hidroxicalamenene }\end{array}$ & P. cassiae & C. spectabilis $(\mathrm{T})$ & $\begin{array}{c}\text { Inhibiting } \\
\text { acetylcholinesterase, } \\
\text { Antifungal }\end{array}$ & [84] \\
\hline 187 & & $\begin{array}{l}\text { (7S,10R)-3-Hidroxicalamen-8- } \\
\text { one }\end{array}$ & P. cassiae & C. spectabilis $(\mathrm{T})$ & $\begin{array}{c}\text { Inhibiting } \\
\text { acetylcholinesterase, } \\
\text { Antifungal }\end{array}$ & [84] \\
\hline
\end{tabular}


Table 1. Cont.

\begin{tabular}{|c|c|c|c|c|c|c|}
\hline Number & Structural Types & Compounds & Strains & $\begin{array}{l}\text { Habitats } \\
\left(\mathrm{T} / \mathbf{M}^{\mathrm{a}}\right)\end{array}$ & Activities & Refs. \\
\hline 188 & & Aristelegone-A & P. cassiae & C. spectabilis $(\mathrm{T})$ & $\begin{array}{l}\text { Inhibiting } \\
\text { acetylcholinesterase, } \\
\text { Antifungal }\end{array}$ & [84] \\
\hline 189 & & Phomoarcherin A & P. archeri & Vanilla albidia $(\mathrm{T})$ & Cytotoxic & [85] \\
\hline 190 & & Phomoarcherin B & P. archeri & V.albidia $(\mathrm{T})$ & $\begin{array}{l}\text { Cytotoxic, } \\
\text { Antimalarial }\end{array}$ & [85] \\
\hline 191 & & Phomoarcherin C & P. archeri & V. albidia $(\mathrm{T})$ & Cytotoxic & [85] \\
\hline 192 & & Kampanol A & P. archeri & $V$. albidia $(\mathrm{T})$ & Cytotoxic & [85] \\
\hline 193 & & (+)-S-1-Methyl-abscisic-6-acid & P. amygdali & Call midge $(\mathrm{T})$ & Antibacterial & [86] \\
\hline 194 & & $(+)-S-$ Abscisic acid & P. amygdali & C. midge $(\mathrm{T})$ & Antibacterial & [86] \\
\hline 195 & & $\begin{array}{l}\text { 7-Hydroxy-10- } \\
\text { oxodehydrodihydrobotrydial }\end{array}$ & $\begin{array}{l}\text { Phomopsis sp. } \\
\text { TJ507A }\end{array}$ & P.glaucus $(\mathrm{T})$ & Inhibiting BACE1 & [83] \\
\hline 196 & & Curcumol & $\begin{array}{l}\text { P. castaneae- } \\
\text { mollissimae } \\
\text { GQH87 }\end{array}$ & $\begin{array}{l}\text { Artemisia annua } \\
\text { (T) }\end{array}$ & Cytotoxic & [87] \\
\hline 197 & & 9-Hydroxyphomopsidin & $\begin{array}{l}\text { Phomopsis sp. } \\
\text { CAFT69 }\end{array}$ & $\begin{array}{l}\text { E. calophylloides } \\
\text { (T) }\end{array}$ & $\begin{array}{l}\text { Motility inhibitory } \\
\text { and zoosporicidal } \\
\text { potential }\end{array}$ & [48] \\
\hline 198 & & Phomopsidin & $\begin{array}{l}\text { Phomopsis sp. } \\
\text { CAFT69 }\end{array}$ & $\begin{array}{l}\text { E. calophylloides } \\
\qquad \text { (T) }\end{array}$ & $\begin{array}{l}\text { Motility inhibitory } \\
\text { and zoosporicidal } \\
\text { potential }\end{array}$ & [48] \\
\hline 199 & & AA03390 & $\begin{array}{l}\text { P. lithocarpus } \\
\text { FS508 }\end{array}$ & Sediment (M) & Cytotoxic & [70] \\
\hline 200 & Diterpenoids & Libertellenone J & Phomopsis sp. S12 & $\begin{array}{l}\text { Illigera rhodantha } \\
\text { (T) }\end{array}$ & Anti-inflammatory & [88] \\
\hline 201 & & Libertellenone C & Phomopsis sp. S12 & $-b$ & Anti-inflammatory & [89] \\
\hline 202 & & Libertellenone $\mathrm{T}$ & Phomopsis sp. S12 & $-b$ & Anti-inflammatory & [89] \\
\hline 203 & & Pedinophyllol K & Phomopsis sp. S12 & $-b$ & Anti-inflammatory & [89] \\
\hline 204 & & Pedinophyllol L & Phomopsis sp. S12 & $-b$ & Anti-inflammatory & [89] \\
\hline 205 & & Fusicoccin J & P. amygdali & C. midge $(\mathrm{T})$ & Antibacterial & [86] \\
\hline 206 & & $3 \alpha$-Hydroxyfusicoccin J & P. amygdali & C. midge $(\mathrm{T})$ & Antibacterial & [86] \\
\hline 207 & Triterpenoids & $\begin{array}{l}\text { 3S,22R,26-Trihydroxy- } 8,24 E- \\
\text { euphadien-11-one }\end{array}$ & P. chimonanthi & $\begin{array}{l}\text { Tamarix chinensis } \\
\text { (T) }\end{array}$ & Cytotoxic & [90] \\
\hline 208 & & Betulinic acid & $\begin{array}{c}\text { Phomopsis sp. } \\
\text { SNB-LAP1-7-32 } \\
P .\end{array}$ & $\begin{array}{c}\text { Diospyros } \\
\text { carbonaria }(\mathrm{T})\end{array}$ & Antiviral, Cytotoxic & [91] \\
\hline 209 & & Oleanolic acid & $\begin{array}{l}\text { castaneae-mollissi } \\
\text { mae GQH87 }\end{array}$ & A. аппиа $(\mathrm{T})$ & Cytotoxic & [87] \\
\hline 210 & Steroids & $\begin{array}{c}(14 \beta, 22 E)-9,14- \\
\text { Dihydroxyergosta- } 4,7,22- \\
\text { triene-3,6-dione } \\
(5 \alpha, 6 \beta, 15 \beta, 22 E)-6-\text { Ethoxy- }\end{array}$ & Phomopsis sp. & A. carmichaeli $(\mathrm{T})$ & Antifungal & [92] \\
\hline 211 & & $\begin{array}{l}\text { 5,15-dihydroxyergosta-7,22- } \\
\text { dien-3-one }\end{array}$ & Phomopsis sp. & A. carmichaeli $(\mathrm{T})$ & Antifungal & [92] \\
\hline 212 & & Calvasterol A & Phomopsis sp. & A. carmichaeli $(\mathrm{T})$ & Antifungal & [92] \\
\hline 213 & & Calvasterol B & Phomopsis sp. & A. carmichaeli $(\mathrm{T})$ & Antifungal & [92] \\
\hline 214 & & Ganodermaside D & Phomopsis sp. & A. carmichaeli $(\mathrm{T})$ & Antifungal & [92] \\
\hline 215 & & Dankasterone A & $\begin{array}{c}\text { Phomopsis sp. YM } \\
355364\end{array}$ & A. carmichaeli $(\mathrm{T})$ & $\begin{array}{c}\text { Antifungal, } \\
\text { Anti-influenza }\end{array}$ & [71] \\
\hline 216 & & $\begin{array}{c}3 \beta, 5 \alpha, 9 \alpha \text {-Trihydroxy- } \\
(22 E, 24 R) \text {-ergosta-7,22-dien-6- } \\
\text { one }\end{array}$ & $\begin{array}{l}\text { Phomopsis sp. YM } \\
355364\end{array}$ & A. carmichaeli $(\mathrm{T})$ & Antifungal & [71] \\
\hline 217 & & Phomopsterone B & $\begin{array}{l}\text { Phomopsis sp. } \\
\text { TJ507A }\end{array}$ & P.glaucus $(\mathrm{T})$ & Anti-inflammatory & [93] \\
\hline 218 & & Cyathisterol & $\begin{array}{c}\text { Phomopsis sp. YM } \\
355364\end{array}$ & A. carmichaelii $(\mathrm{T})$ & Antifungal & [26] \\
\hline 219 & Macrolides & Sch-642305 & $\begin{array}{l}\text { Phomopsis sp. } \\
\text { CMU-LMA }\end{array}$ & $\begin{array}{c}\text { Alpinia } \\
\text { malaccensis }(\mathrm{T})\end{array}$ & $\begin{array}{c}\text { Cytotoxic, } \\
\text { Antimicrobial }\end{array}$ & [94] \\
\hline 220 & & LMA-P1 & $\begin{array}{l}\text { Phomopsis sp. } \\
\text { CMU-LMA }\end{array}$ & A. malaccensis $(\mathrm{T})$ & Cytotoxic & [94] \\
\hline 221 & & Benquoine & $\begin{array}{l}\text { Phomopsis sp. } \\
\text { CMU-LMA }\end{array}$ & A. malaccensis $(\mathrm{T})$ & $\begin{array}{c}\text { Cytotoxic, } \\
\text { Antimicrobial }\end{array}$ & [94] \\
\hline 222 & & Aspergillide C & $\begin{array}{l}\text { Phomopsis sp. } \\
\text { IFB-ZS1-S4 }\end{array}$ & S. hainanensis (M) & $\begin{array}{l}\text { Inhibiting } \\
\text { neuraminidase }\end{array}$ & [37] \\
\hline
\end{tabular}


Table 1. Cont.

\begin{tabular}{|c|c|c|c|c|c|c|}
\hline Number & Structural Types & Compounds & Strains & $\begin{array}{c}\text { Habitats } \\
\left(\mathrm{T} / \mathrm{M}^{\mathrm{a}}\right)\end{array}$ & Activities & Refs. \\
\hline 223 & & Lithocarpin A & $\begin{array}{l}\text { P. lithocarpus } \\
\text { FS508 }\end{array}$ & Sediment (M) & Cytotoxic & [95] \\
\hline 224 & & Lithocarpin B & $\begin{array}{l}\text { P. lithocarpus } \\
\text { FS508 }\end{array}$ & Sediment (M) & Cytotoxic & [95] \\
\hline 225 & & Lithocarpin C & $\begin{array}{l}\text { P. lithocarpus } \\
\text { FS508 }\end{array}$ & Sediment (M) & Cytotoxic & [95] \\
\hline 226 & & Lithocarpin D & $\begin{array}{l}\text { P. lithocarpus } \\
\text { FS508 }\end{array}$ & Sediment (M) & Cytotoxic & [95] \\
\hline 227 & \multirow[t]{16}{*}{ Alkaloids } & Phomopchalasin B & Phomopsis sp. shj2 & $\begin{array}{l}\text { I. eriocalyx var. } \\
\text { laxiflora }(\mathrm{T})\end{array}$ & Antimigratory & [96] \\
\hline 228 & & Phomopsichalasin G & $\begin{array}{c}P . \text { spp. xy } 21 \text { and } \\
\text { xy } 22\end{array}$ & X. granatum $(\mathrm{M})$ & Cytotoxic & [97] \\
\hline 229 & & 18-Metoxycytochalasin J & Phomopsis sp. & Garcinia kola $(\mathrm{T})$ & $\begin{array}{c}\text { Cytotoxic, } \\
\text { Antibacterial } \\
\text { Cytotoxic, }\end{array}$ & [98] \\
\hline 230 & & Cytochalasin $\mathrm{H}$ & $\begin{array}{l}\text { Phomopsis sp. } \\
\text { Phomopsis sp. } \\
\text { By } 254 \\
\text { Phomopsis sp. }\end{array}$ & $\begin{array}{c}\text { G. kola }(\mathrm{T}) \\
\text { Gossypium } \\
\text { hirsutum }(\mathrm{T}) \\
\text { S. spectabilis }(\mathrm{T})\end{array}$ & $\begin{array}{c}\text { Antibacterial } \\
\text { Antifungal } \\
\text { Inhibiting } \\
\text { acetylcholinesterase, } \\
\text { Anti-inflammatory }\end{array}$ & $\begin{array}{l}{[98]} \\
{[99]} \\
{[49]}\end{array}$ \\
\hline 231 & & Cytochalasin J & $\begin{array}{l}\text { Phomopsis sp. } \\
\text { Phomopsis sp. } \\
\text { P. asparagi }\end{array}$ & $\begin{array}{c}\text { G. kola }(\mathrm{T}) \\
\text { S. spectabilis }(\mathrm{T}) \\
\text { Peperomia sui }(\mathrm{T})\end{array}$ & $\begin{array}{c}\text { Cytotoxic, } \\
\text { Antibacterial } \\
\text { Anti-inflammatory } \\
\text { Antiandrogen }\end{array}$ & $\begin{array}{l}{[98]} \\
{[49]} \\
{[100]}\end{array}$ \\
\hline 232 & & Phomopchalasin C & Phomopsis sp. shj2 & $\begin{array}{l}\text { I. eriocalyx var. } \\
\text { laxiflora }(\mathrm{T})\end{array}$ & $\begin{array}{c}\text { Cytotoxic, } \\
\text { Anti-inflammatory, } \\
\text { Antimigratory }\end{array}$ & [96] \\
\hline 233 & & Cytochalasin N & $\begin{array}{l}\text { Phomopsis sp. } \\
\text { By254 }\end{array}$ & G. hirsutum $(\mathrm{T})$ & Antifungal & [99] \\
\hline 234 & & Epoxycytochalasin $\mathrm{H}$ & $\begin{array}{c}\text { Phomopsis sp. } \\
\text { By254 }\end{array}$ & G. hirsutum $(\mathrm{T})$ & Antifungal & [99] \\
\hline 235 & & Diaporthalasin & $\begin{array}{l}\text { Phomopsis sp. } \\
\text { PSU-H188 }\end{array}$ & H. brasiliensis $(\mathrm{T})$ & Anti-MRSA & [73] \\
\hline 236 & & (+)-Tersone E & P. tersa FS441 & Sediment (M) & $\begin{array}{c}\text { Antibacterial, } \\
\text { Cytotoxic }\end{array}$ & [101] \\
\hline 237 & & ent-Citridone A & P. tersa FS441 & Sediment (M) & Antibacterial & [101] \\
\hline 238 & & Phochrodine C & Phomopsis sp. 33\# & R. stylosa (M) & Anti-inflammatory & [102] \\
\hline 239 & & Phochrodine D & Phomopsis sp. 33\# & R. stylosa (M) & $\begin{array}{c}\text { Anti-inflammatory, } \\
\text { Antioxidant }\end{array}$ & [102] \\
\hline 240 & & PM181110 & P. glabrae & $\begin{array}{l}\text { Pongamia pinnata } \\
\text { (T) }\end{array}$ & Anticancer & [103] \\
\hline 241 & & Fusaristatin A & P. longicolla S1B4 & $-b$ & Antibacterial & [34] \\
\hline 242 & & Exumolide A & $\begin{array}{c}\text { Phomopsis sp. (No. } \\
\text { ZH-111) }\end{array}$ & Sediment (M) & $\begin{array}{l}\text { Accelerating the } \\
\text { growth of SIV branch, } \\
\text { Cytotoxic }\end{array}$ & [44] \\
\hline 243 & \multirow[t]{4}{*}{ Flavonoids } & Quercetin & $\begin{array}{l}\text { P. castaneae- } \\
\text { mollissimae } \\
\text { GQH87 }\end{array}$ & A. annua $(\mathrm{T})$ & Cytotoxic & [87] \\
\hline 244 & & Luteolin & $\begin{array}{l}\text { P. castaneae- } \\
\text { mollissimae } \\
\text { GQH87 }\end{array}$ & A. anпиа $(\mathrm{T})$ & Cytotoxic & [87] \\
\hline 245 & & Naringenin & $\begin{array}{l}\text { P. castaneae- } \\
\text { mollissimae } \\
\text { GQH87 }\end{array}$ & A. аппиа $(\mathrm{T})$ & Cytotoxic & [87] \\
\hline 246 & & Luteolin-7-O-glucoside & $\begin{array}{l}\text { P. castaneae- } \\
\text { mollissimae } \\
\text { GQH87 }\end{array}$ & A. annua $(\mathrm{T})$ & Cytotoxic & [87] \\
\hline
\end{tabular}

${ }^{\mathrm{a}}$ T: terrestrial environment; M: marine environment; ${ }^{\mathrm{b}}$ The habitat was not mentioned.

\subsection{Polyketides}

Polyketides are a large and diverse family of natural products, containing various chemical structures and biological activities [104]. In this review, 171 polyketides are summarized from Phomopsis, accounting for $70 \%$ of the total compounds from Phomopsis. 
The main bioactivities involve cytotoxic, antibacterial and antifungal activities. Herein, we classify these polyketides into xanthones, chromones, chromanones, benzofuranones, pyrones, quinones, phenols, oblongolides, and unclassified polyketides.

\subsubsection{Xanthones}

Xanthones are a kind of compounds with the framework of 9H-xanthen-9-one, which mainly have anti-inflammatory, antimicrobial, antioxidant and cytotoxic activities [105]. A series of xanthones were obtained from the fermentation products of Phomopsis sp. isolated from Paris polyphylla var. yunnanensis, including three new compounds, 1,5-dihydroxy-3hydroxyethyl-6-methoxycarbonylxanthone (1), 1-hydroxy-5-methoxy-3-hydroxyethyl-6methoxycarbonylxanthone (2), 1-hydroxy-3-hydroxyethyl-8-ethoxy-carbonyl-xanthone (3), and seven known ones, pinselin (4), 1-hydroxy-8-(hydroxymethyl)-3-methoxy-6methylxanthone (5), secosterigmatocystin (17), 1,7-dihydroxy-2-methoxy-3-(3-methylbut-2enyl)xanthone (22), 1-hydroxy-4,7-dimethoxy-6-(3-oxobutyl)xanthone (23), asperxanthone (24) and 6-O-methyl-2-deprenylrheediaxanthone B (25). The cytotoxicities of all compounds to five human tumor cells (NB4, A549, SHSY5Y, PC3, and MCF7) were evaluated by using paclitaxel as positive control. The results showed that compounds $\mathbf{1}$ and $\mathbf{3}$ displayed cytotoxic activities and provided the $\mathrm{IC}_{50}$ values of 3.6 and $2.5 \mu \mathrm{M}$ against $\mathrm{A} 549$ cells, and $\mathbf{1}$ gave an $\mathrm{IC}_{50}$ value of $2.7 \mu \mathrm{M}$ against MCF7 cells. Compounds 22-23 showed weak activities and offered $\mathrm{IC}_{50}$ values greater than $10 \mu \mathrm{M}$ for five tested cells. The others gave $\mathrm{IC}_{50}$ values between 3.8-10 $\mu \mathrm{M}$ against tested cells [23]. A new compound, 2,6dihydroxy-3-methyl-9-oxoxanthene-8-carboxylic acid methyl ester (6), was isolated from Phomopsis sp. (No. SK7RN3G1) of mangrove sediment in the Shankou, Hainan, China. It showed cytotoxicity towards HEp-2 $\left(\mathrm{IC}_{50}=8 \mu \mathrm{g} / \mathrm{mL}\right)$ and HepG2 $\left(\mathrm{IC}_{50}=9 \mu \mathrm{g} / \mathrm{mL}\right)$ cancer cells [24]. Three secondary metabolites were characterized from fermentation products of $P$. amygdali, isolated from Paris axialis: 4,5-dihydroxy-3-(2-hydroxyethyl)-1-methoxy-8methoxycarbonylxanthone (7), 1,8-dihydroxy-4-(2-hydroxyethyl)-3-methoxyxanthone (8), and paucinervin E (13). Compound 7 was active against $\mathrm{A} 549\left(\mathrm{IC}_{50}=2.6 \mu \mathrm{M}\right)$ and PC3 $\left(\mathrm{IC}_{50}=2.4 \mu \mathrm{M}\right)$ cell lines. Compounds 8 and $\mathbf{1 3}$ displayed moderate activities with $\mathrm{IC}_{50}$ values in the range of 5.2-9.2 $\mu \mathrm{M}$ against one or more cell lines of NB4, A549, SHSY5Y, PC3 and MCF7 [25]. Hydroxyvertixanthone (9) was obtained from the endophytic fungus Phomopsis sp. YM 355364, originated from Chinese medicinal plant Aconitum carmichaelii. It showed antimicrobial activity with minimal inhibitory concentration (MIC) values of 256, 256, 128, and $64 \mu \mathrm{g} / \mathrm{mL}$ against Escherichia coli, Bacillus subtilis, Pyricularia oryzae, and Candida albicans, respectively [26]. The fermentation of fungus Phomopsis sp. derived from Paris daliensis, led to the isolation of six xanthones and identified as dalienxanthones A-C (10-12), 3,8-dihydroxy-4-(2,3-dihydroxy-1-hydroxymethylpropyl)-1-methoxyxanthone (18), oliganthins $\mathrm{E}$ (19), and cratoxylumxanthone $\mathrm{D}$ (26). These compounds were evaluated for cytotoxicities of five cancer cell lines (NB4, A549, SHSY5Y, PC3 and MCF-7). Compounds 12 and 18 were active to SHSY5Y with $\mathrm{IC}_{50}$ values of 3.8 and $3.5 \mu \mathrm{M}$, respectively, and the remaining compounds provided $\mathrm{IC}_{50}$ values in the range of 4.6-9.2 $\mu \mathrm{M}$ [27]. An investigation of extracts from fungus $P$. amygdali derived from the rhizome of Paris polyphylla var. yunnanensis afforded a new xanthone, 1,3-dihydroxy-4-(1,3,4-trihydroxybutan-2-yl)-8methoxy-9H-xanthen-9-one (14). The bioactive results showed that 14 exhibited significant cytotoxic activity against $\mathrm{A} 549\left(\mathrm{IC}_{50}=5.8 \mu \mathrm{M}\right)$ and PC3 $\left(\mathrm{IC}_{50}=3.6 \mu \mathrm{M}\right)[28]$.

An endophytic fungus P. amygdali associated with the rhizome of Paris axialis was cultured to obtain five xanthones: 3-methoxy-1,4,8-trihydroxy-5-(1', $3^{\prime}, 4^{\prime}$-trihydroxybutan-2'yl)-xanthone (15), 8-methoxy-1,3,4-trihydroxy-5-(1', $3^{\prime}, 4^{\prime}$-trihydroxybutan-2'-yl)-xanthone (16), secosterigmatocystin (17), dihydrosterigmatocystin (20), and vieillardixanthone (21). The cytotoxic assay for NB4, A549, SHSY5Y, PC3 and MCF7 cancer cells were evaluated. The $\mathrm{IC}_{50}$ values of compound $\mathbf{1 5}$ against A549 and $\mathbf{1 6}$ against SHSY5Y were 3.6 and $4.2 \mu \mathrm{M}$, respectively. Compounds $\mathbf{1 7}$ and 20-21 displayed moderate activities with $\mathrm{IC}_{50}$ values in the range of 5.4-8.8 $\mu \mathrm{M}$ [29]. Studies of an endophytic fungus Phomopsis sp. (ZH76) from the stems of the mangrove tree Excoecaria agallocha contained a new O-glycoside 
compound, 3-O-(6-O- $\alpha$-L-arabinopyranosyl)- $\beta$-D-glucopyranosyl-1,4-dimethoxyxanthone (27). The $\mathrm{IC}_{50}$ values of cytotoxicity for compound 27 on HEp-2 and HepG2 cells were 9 and $16 \mu \mathrm{mol} / \mathrm{mL}$, respectively [30]. Phomoxanthone A (28), a dimeric tetrahydroxanthone, was extracted from P. longicolla of the mangrove tree Sonneratia caseolaris. Compound 28 had the strongest pro-apoptotic activity on human cancer cell lines and cisplatin-resistant cells, and its activity on healthy blood cells was reduced by more than 100 times. It was the most effective activator of mouse T lymphocytes, NK cells, and macrophages [31]. The study on secondary metabolites from fungus Phomopsis sp. IM 41-1 of mangrove plant Rhizhopora mucronata afforded phomoxanthone A (28) and 12-O-deacetyl-phomoxanthone A (29). When the concentration was $30 \mu \mathrm{g} /$ disk, compounds 28 and 29 showed moderate antimicrobial activities against Botrytis cinerea, Sclerotinia sclerotiorum, Diaporthe medusaea, and Staphylococcus aureus, but were inactive against Pseudomonas aeruginosa [32]. Four bioactive metabolites, dicerandrols A-C (30-32) and deacetylphomoxanthone B (33), were derived from $P$. longicolla S1B4. All compounds exhibited strong antibacterial activities against Xanthomonas oryzae KACC 10331. Dicerandrol A (30) also displayed notable antimicrobial activity against $S$. aureus, B. subtilis, and C. albicans with MIC values of 0.25 , 0.125 and $2 \mu \mathrm{g} / \mathrm{mL}$ [34]. Phomopsis sp. HNY29-2B, isolated from mangrove plant Acanthus ilicifolius, produced four xanthone derivatives, 30-31, 33 and penexanthone A (34). Compounds 30-31 and 33-34 displayed cyctotoxicities and provided $\mathrm{IC}_{50}$ values of 1.7642.82 $\mu$ M against MDA-MB-435, HCT-116, Calu-3, Huh7, and MCF-10A human cancer cell lines [35]. The structures of xanthones (1-34) are shown in Figure 1.

\subsubsection{Chromones}

Chromones are a class of bioactive compounds with a benzo- $\gamma$-pyrone skeleton, which have been reported to have various activities, such as anti-tumor, anti-viral, antimicrobial, anti-inflammatory, and antioxidant [106]. Phomopsis sp. 33\#, a mangrove endophytic fungus isolated from the bark of Rhizophora stylosa, produced four new chromone derivatives, (+)-phomopsichin A (35), (-)-phomopsichin B (36), phomopsichins C (37) and D (38), along with a known phomoxanthone $A(28)$. These metabolites displayed low effects on inhibitions of acetylcholinesterase and $\alpha$-glucosidase, radical scavenging function on DPPH and $\mathrm{OH}$, and antimicrobial activities [33]. A cytotoxic chromone, chaetocyclinone B (39), was characterized from a culture of Phomopsis sp. HNY29-2B, an endophytic fungus obtained from the mangrove plant $A$. ilicifolius Linn. Compound 39 had cytotoxic activity against PC-3 $\left(\mathrm{IC}_{50}=8.13 \mu \mathrm{mol} / \mathrm{L}\right)$ and DU145 $\left(\mathrm{IC}_{50}=3.59 \mu \mathrm{mol} / \mathrm{L}\right)$ [36]. The fungus Phomopsis sp. IFB-ZS1-S4 isolated from Scaevola hainanensis Hance extracted a known pestalotiopsone $\mathrm{F}(40)$, which showed moderate inhibition on neuraminidase in vitro with $\mathrm{IC}_{50}$ value of $9.90 \pm 0.42 \mu \mathrm{M}$ [37]. Cultivation of Phomopsis sp. xy21 derived from the mangrove Xylocarpus granatum afforded a new xanthone-derived polyketide, phomoxanthone $F$ (41). It showed inhibitory effects on VSV-G pseudotyped viral supernatant (HIV-1) with the inhibitory rate of $16.48 \pm 6.67 \%$ at a concentration of $20 \mu \mathrm{M}$, which was higher than that of the positive control, efavirenz with a rate of $88.54 \pm 0.45 \%$ [38]. 5-Hydroxy-3-hydroxymethyl2-methyl-7-methoxychromone (42) was separated from the extracts of Phomopsis sp. (No. Gx-4) derived from mangrove sediment in ZhuHai, Guangdong, China. It showed low cytotoxic activity with $\mathrm{IC}_{50}$ values greater than $50 \mu \mathrm{mol} / \mathrm{mL}$ towards Hep-2 and HepG2. Moreover, it also significantly inhibited the growth of subintestinal vessel plexus (SIV) branches [39]. According to the bioassay-guided fractionation, two new chromones, phomochromones A (43) and B (44) were obtained from an endophytic fungus Phomopsis sp. of Cistus monspeliensis. They displayed remarkable antifungal, antibacterial, and antialgal activities against Microbotryum violaceum, E. coli, Bacillus megaterium, and Chlorella fusca [40]. Chemical investigation of Phomopsis sp. CGMCC No. 5416 isolated from Achyranthes bidentata led to the identification of two novel chromanones, phomochromanones A (45) and B (46). They showed anti-HIV activities with $\mathrm{IC}_{50}$ values of 20.4 and $32.5 \mu \mathrm{g} / \mathrm{mL}$, and exhibited moderate cytotoxic activities towards A549, MDA-MB-231, and PANC-1 with $\mathrm{CC}_{50}$ values between $62.5-79.3 \mu \mathrm{g} / \mathrm{mL}$ [41]. A new naphtho- $\gamma$-pyrone compound, 5- 
hydroxy-6,8-dimethoxy-2-benzyl-4H-naphtho[2,3-b]-pyran-4-one (47), was obtained from Phomopsis sp. ZSU-H26 of the mangrove tree E. agallocha. This compound showed cytotoxic activity against HEp-2 $\left(\mathrm{IC}_{50}=10 \mu \mathrm{g} / \mathrm{mL}\right)$ and HepG2 $\left(\mathrm{IC}_{50}=8 \mu \mathrm{g} / \mathrm{mL}\right)$ [42]. The following work on the similar strain Phomopsis sp. (\#ZSU-H76) from the same host additionally obtained phomopsis-H76 A (48), which significantly promoted the growth of the branches of SIV [43]. The structures of chromones (35-48) are shown in Figure 2.

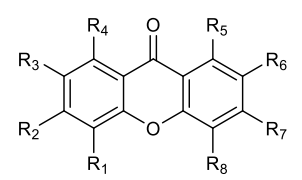

$\begin{array}{llllllll} & \mathrm{R}_{1} & \mathrm{R}_{2} & \mathrm{R}_{3} & \mathrm{R}_{4} & \mathrm{R}_{5} & \mathrm{R}_{6} & \mathrm{R}_{7} \\ \mathbf{1} & \mathrm{OH} & \mathrm{COOCH}_{3} & \mathrm{H} & \mathrm{H} & \mathrm{OH} & \mathrm{H} & \mathrm{CH}_{2} \mathrm{CH}_{2} \mathrm{OH} \\ \mathbf{2} & \mathrm{OH} & \mathrm{COOCH} & \mathrm{H} & \mathrm{H} & \mathrm{OCH}_{3} & \mathrm{H} & \mathrm{CH}_{2} \mathrm{CH}_{2} \mathrm{OH} \\ 3 & \mathrm{H} & \mathrm{H} & \mathrm{H} & \mathrm{COOCH}_{3} & \mathrm{OH} & \mathrm{H} & \mathrm{CH}_{2} \mathrm{CH}_{2} \mathrm{OH} \\ \mathbf{4} & \mathrm{H} & \mathrm{H} & \mathrm{OH} & \mathrm{COOCH}_{3} & \mathrm{OH} & \mathrm{H} & \mathrm{CH}_{3} \\ \mathbf{5} & \mathrm{H} & \mathrm{CH}_{3} & \mathrm{H} & \mathrm{OH} & \mathrm{CH}_{2} \mathrm{OH} & \mathrm{H} & \mathrm{OCH}_{3} \\ \mathbf{6} & \mathrm{H} & \mathrm{OH} & \mathrm{H} & \mathrm{COOCH}_{3} & \mathrm{H} & \mathrm{OH} & \mathrm{CH}_{3} \\ 7 & \mathrm{OH} & \mathrm{CH}_{2} \mathrm{CH}_{2} \mathrm{OH} & \mathrm{H} & \mathrm{OCH}_{3} & \mathrm{COOCH} & \mathrm{H} & \mathrm{H} \\ \mathbf{8} & \mathrm{H} & \mathrm{H} & \mathrm{H} & \mathrm{OH} & \mathrm{OH} & \mathrm{H} & \mathrm{OCH}_{3} \\ \mathbf{9} & \mathrm{H} & \mathrm{H} & \mathrm{H} & \mathrm{OH} & \mathrm{COOCH} & \mathrm{OH} & \mathrm{H}\end{array}$
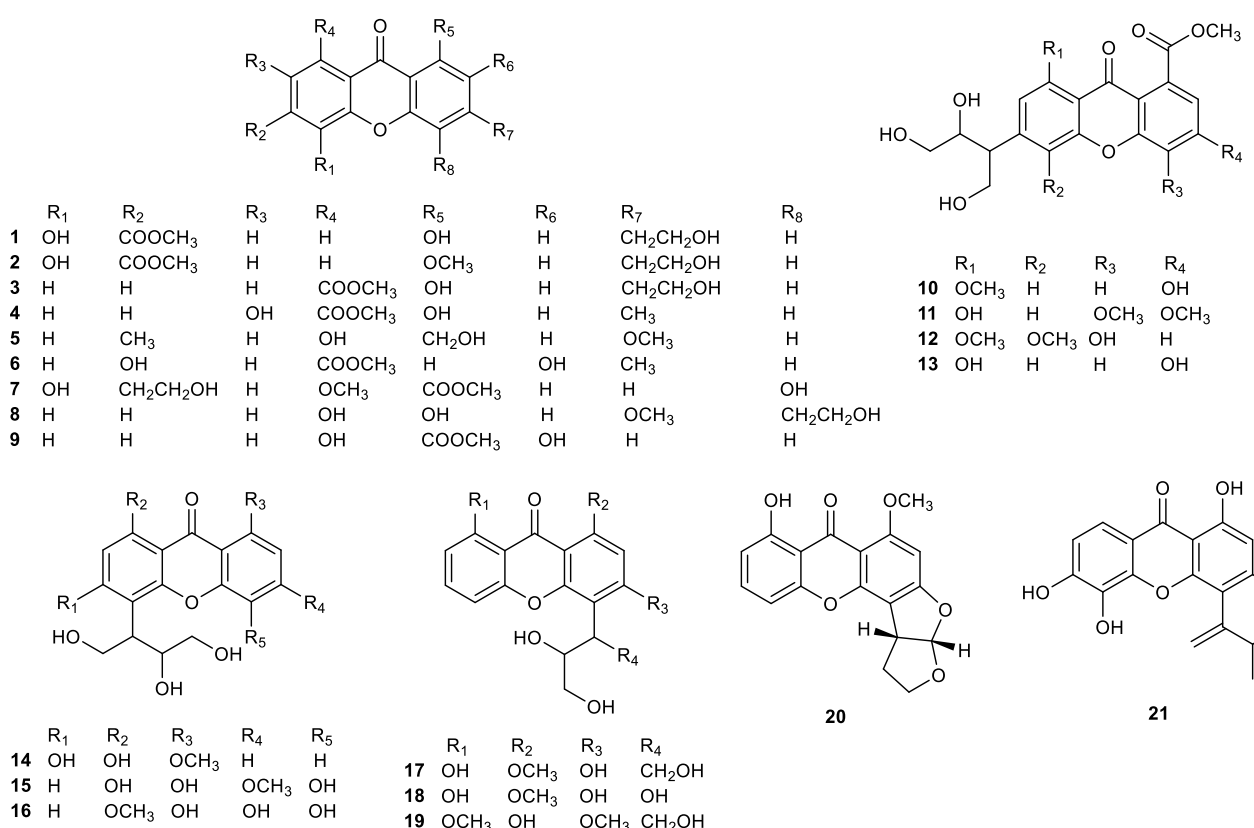
$17 \begin{array}{llll}\mathrm{R}_{1} & \mathrm{R}_{2} & \mathrm{R}_{3} & \mathrm{R}_{4} \\ \mathrm{OH} & \mathrm{OCH}_{3} & \mathrm{OH} & \mathrm{CH}_{2} \mathrm{OH}\end{array}$ $18 \mathrm{OH} \mathrm{OCH}_{3} \mathrm{OH} \quad \mathrm{OH}$<smiles>COc1cc2c(c3oc4cccc(O)c4c(=O)c13)[C@H]1CCO[C@@H]1O2</smiles>

20<smiles>C=C(c1c(OC)cc(O)c2c(=O)c3ccc(O)c(O)c3oc12)C(C)C</smiles>

21<smiles>[Z10]Oc1ccc2c(=O)c3c(O)cc4c(c3oc2c1O)C(C)(C)C(C)O4</smiles>

24<smiles>[R6][R6](=O)OC(C)=O</smiles>

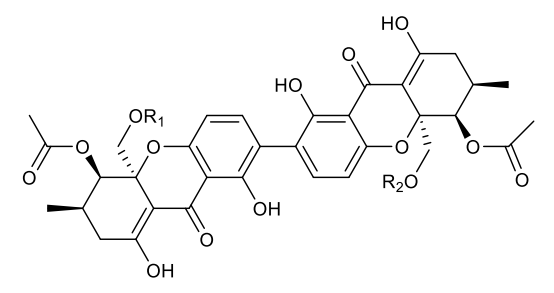

$30 \mathrm{R}_{1}=\mathrm{R}_{2}=\mathrm{H}$ $31 R_{1}=A c R_{2}=H$
$32 R_{1}=A c \quad R_{2}=A c$<smiles>COc1cc2c(=O)c3c(O)ccc(OC)c3oc2cc1CCC(C)=O</smiles>

23<smiles>CCCCOC1OC2OC(Oc3cc(OC)c4c(=O)c5ccccc5oc4c3OC)C1OC1OC2C(O)C1O</smiles><smiles>COc1c(CC=C(C)C)c(O)cc2oc3cc(O)c4c(c3c(=O)c12)CC(C(C)(C)O)O4</smiles>

26 ${ }_{27} \mathrm{OH}$<smiles>[R]CO[C@]12Oc3ccc(-c4ccc(O)c5c4O[C@@]4(CO)C(=C(O)C[C@H](C)[C@H]4OC(C)=O)C5=O)c(O)c3C(=O)C1=C(O)C[C@@H](C)[C@H]2OC(C)=O</smiles>

Figure 1. Chemical structures of compounds 1-34 from Phomopsis. 
<smiles>COC(=O)c1cc(O)cc2oc3c(c(=O)c12)[C@H](OC)O[C@H](C)C3</smiles>

35<smiles>COC(=O)c1cc(OC)c(O)c2oc3c(c(=O)c12)COC(C)=C3</smiles>

39<smiles>COC(=O)c1cc(OC)c(O)c2c(=O)c3c(oc12)C[C@@H](C)O[C@H]3OC</smiles>

36<smiles>CCCCCc1cc(=O)c2c(CC(=O)OC)cc(O)cc2o1</smiles><smiles>COC(=O)c1cc(OC)c(O)c2c(=O)c3c(oc12)C[C@@H](C)OC3</smiles>

37<smiles>CC1CC(=O)O[C@H]1c1oc2cccc(O)c2c(=O)c1O</smiles><smiles>COC(=O)c1cc(O)cc2oc(CC(C)O)c(CO)c(=O)c12</smiles><smiles>COc1cc(O)c2c(=O)c(CO)c(C)oc2c1</smiles><smiles>Cc1cc2c(c(C)c1O)O[C@H](C)CC2=O</smiles>

43<smiles>Cc1cc2c(c(C)c1O)O[C@H](C)[C@H](O)C2=O</smiles>

44<smiles>CCC[C@H]1CC(=O)c2cccc(O)c2O1</smiles>

45<smiles>CCCC1CC(=O)c2c(O)ccc(O)c2O1</smiles>

46<smiles>COc1cc(OC)c2c(O)c3c(=O)cc(Cc4ccccc4)oc3cc2c1</smiles>

47<smiles>CC1CC(=O)O[C@@H]1C1(CO)CC(=O)c2c(ccc(-c3ccc4c(c3O)C(=O)C[C@H](CO)O4)c2O)O1</smiles><smiles>C[C@H]1OC(=O)c2c(O)ccc(O)c2[C@H]1O</smiles>

49<smiles>CO[C@H]1c2cccc(O)c2C(=O)O[C@@H]1C</smiles>

50<smiles>[R]c1cc(O)c2c(=O)oc3cc(O)c([R])c(C)c3c2c1</smiles>

$55 \mathrm{R}_{1}=\mathrm{OH} \quad \mathrm{R}_{2}=\mathrm{H}$

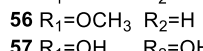<smiles>CC1Cc2c(C(=O)O)ccc(O)c2C(=O)O1</smiles>

51<smiles>Cc1cc(=O)oc2c(O)ccc(O)c12</smiles>

52<smiles>COc1cc(O)c2c(=O)oc(C[C@H](C)O)cc2c1</smiles>

53<smiles>CCCCCCCC1OC(=O)Cc2cc(O)c(O)c(O)c21</smiles>

54<smiles>CC[C@H](C)[C@H]1O[C@@H]2O[C@@H](C)Cc3cc(O)c(C)c(c32)C1=O</smiles>

58

Figure 2. Chemical structures of compounds 35-58 from Phomopsis.

\subsubsection{Chromanones}

Chromanones have been widely studied due to their structural characteristics. They always have important biological and pharmacological activities, including cytotoxic, antimicrobial, antiviral, antioxidant, etc [107]. The culture of a marine fungus Phomopsis sp. (No. $\mathrm{ZH}-111)$ from mangrove sediment of Zhuhai, Guangdong, China, obtained a new isochroman, (3R,4S)-3,4-dihydro-4,5,8-trihydroxy-3-methylisocoumarin (49). It could promote the growth of SIV branches and exhibited low cytotoxic activity against Hep-2 and HepG2 cells with $\mathrm{IC}_{50}$ values above $50 \mathrm{mg} / \mathrm{mL}$ [44]. Three compounds were separated from Phomopsis sp. (No. Gx-4), including (3R,4S)-3,4-dihydro-8-hydroxy-4-methoxy-3-methylisocoumarin (50), 3,4-dihydro-8-hydroxy-3-methyl-1H-2-benzopyran-1-one-5-carboxylic acid (51), and 5,8-dihydroxy-4-methylcoumarin (52). All isolated compounds showed weak cytotoxic activities against Hep-2 and HepG2 cells with $\mathrm{IC}_{50}$ values above $50 \mu \mathrm{mol} / \mathrm{mL}$. In addition, compounds $\mathbf{5 0}$ and $\mathbf{5 1}$ significantly promoted the growth of SIV branches, while $\mathbf{5 2}$ inhibited their growth [39]. The endophytic fungus Phomopsis sp. sh917 found in stems of Isodon eriocalyx var. laxiflora obtained (10S)-diaporthin (53), showing antiangiogenic activity that inhibited the angiogenesis process induced by vascular endothelial growth factor (VEGF) [45]. From agar-supported fermentation culture of Phomopsis sp. CMU-LMA derived from Alpinia malacensis, a trihydroxybenzene lactone, cytosporone D (54) was 
isolated. It showed antimicrobial activity and inhibited E. coli DnaG primase with an $\mathrm{IC}_{50}$ value of $0.25 \mathrm{mM}$ [46]. Alternariol (55) and 5'-hydroxyalternariol (57) were isolated from the endophytic fungus Phomopsis sp. A240 of Taxus chinensis var. mairei. Compound 55 showed low cytotoxicity against SF-268 $\left(\mathrm{IC}_{50}=88.1 \mu \mathrm{M}\right), \mathrm{MCF}-7\left(\mathrm{IC}_{50}=94.36 \mu \mathrm{M}\right)$, and NCI-H460 $\left(\mathrm{IC}_{50}=81.35 \mu \mathrm{M}\right)$. Moreover, compound 57 had antioxidant activity with $\mathrm{IC}_{50}$ values of $42.83 \mu \mathrm{M}$ [47]. Three compounds were sourced from Endodesmia calophylloides associated with Phomopsis sp. CAFT69, including alternariol (55), alternariol-5-O-methyl ether (56) and 5'-hydroxyalternariol (57). In the range of 1-10 $\mu \mathrm{g} / \mathrm{mL}$, compounds 55-57 had certain motility inhibition and lytic activities on the zoospores of grapevine downy mildew pathogen P. viticola in dose- and time-dependent manner [48]. Phomochromanone C (58) was extracted from Phomopsis sp. CGMCC No. 5416. The bioactivity assay revealed that compound 58 showed cytotoxicity towards A549, MDA-MB-231, and PANC-1 with $\mathrm{CC}_{50}$ values of $69.4,53.5$, and $36.5 \mu \mathrm{g} / \mathrm{mL}$, and it induced early apoptosis of PANC-1 cancer cells with the rate of $10.52 \%$ [41]. The structures of chromanones (49-58) are shown in Figure 2.

\subsubsection{Benzofuranones}

Benzofuranones are an important intermediate of pharmacophores and drug molecules in natural products. Due to the furan ring being unstable and easy to open and crack, benzofuranones as a pharmaceutical intermediate have been widely concerned by pharmaceutical chemists [108]. The endophytic fungus Phomopsis sp. A123 isolated from mangrove plant Kandelia candel (L.) Druce, produced a novel depsidone, phomopsidone A (66), a known excelsione (67), and four known isobenzofuranones (59-62). All compounds showed different degrees of cytotoxicities against Raji and MDA-MB-435 tumor cells with $\mathrm{IC}_{50}$ values above $18 \mu \mathrm{M}$, displayed low antioxidant activities through DPPH radical scavenging effects, and exhibited antifungal activities [50]. The research on bioactive metabolites of marine fungus Phomopsis sp. (No. ZH-111) led to the isolation of 4-(hydroxymethyl)-7- methoxy-6methyl-1(3H)-isobenzofuranone (63). Compound 63 inhibited the growth of SIV branches and exhibited low cytotoxic activity with $\mathrm{IC}_{50}$ values above $50 \mathrm{mg} / \mathrm{mL}$ against Hep-2 and HepG2 cells [44]. Chemical investigations of secondary metabolites from Phomopsis sp. BCC 45011 of $X$. granatum resulted in the identification of two known metabolites, cytosporones E (64) and P (65). Compounds 64 and 65 showed antimalarial activities against Plasmodium falciparum $\mathrm{K} 1$ with $\mathrm{IC}_{50}$ values of 2.02 and $3.65 \mu \mathrm{g} / \mathrm{mL}$, and 64 exhibited cytotoxicity against MCF-7, NCI-H187, and Vero cells with IC $_{50}$ values at $29.66,5.84$, and $4.53 \mu \mathrm{g} / \mathrm{mL}$, respectively [51]. Cultivation of Phomopsis sp. CAFT69 afforded excelsional (68). In the range of $1-10 \mu \mathrm{g} / \mathrm{mL}$, compound 68 had certain motility inhibition and lytic activities on the zoospores of grapevine downy mildew pathogen $P$. viticola in dose- and time-dependent manner [48]. Lithocarols A-F (69-74), with highly-oxygenated isobenzofuran skeleton, and isoprenylisobenzofuran A (75), were derived from P. lithocarpus FS508 isolated from a deep-sea sediment collected from the Indian Ocean. These metabolites were cytotoxic and provided $\mathrm{IC}_{50}$ values between 10.5-87.7 $\mu \mathrm{M}$ against HepG-2, MCF-7, SF-268, and A549 cells [52]. The endophytic fungus Phomopsis sp., separated from Paris polyphylla var. yunnanensis, gave three new arylbenzofurans (76-78) and four known compounds, moracin $\mathrm{N}(79)$, 2-(2'-methoxy-4'-hydroxy)-aryl-3-methy-6-hydroxybenzofuran (80), iteafuranal B (81), and moracin P (82). Compounds 76-82 showed inhibitory effects on tobacco mosaic virus (TMV) with inhibition rates of 18.6-35.2\% [53]. The structures of benzofuranones (59-82) are shown in Figure 3.

\subsubsection{Pyrones}

Pyrones are a kind of polyketides with six membered oxygen-containing heterocycles. As the precursor of many plants, animals, and microorganisms' biosynthetic reactions, as well as its outstanding anti-tumor and antibacterial activities, researchers have shown strong interest [109]. Eight compounds were identified from the strain $P$. asparagi SWUKJ5.2020 isolated from medicinal plant Kadsura angustifolia, including five 
new 2-pyrone compounds, phomaspyrones A-E (83 and 85-88), along with three known metabolites, macommelin-8,9-diol (84), macommelin-9-ol (89), and macommelin (90). All isolated metabolites showed significant cytotoxic activities against six tested tumor cells (A549, Raji, HepG2, MCF-7, HL-60 and K562) with IC 50 values of 1.0-26.8 $\mu \mathrm{g} / \mathrm{mL}$. However, phomaspyrone $\mathrm{C}$ (86) display better activity than the other compounds with $\mathrm{IC}_{50}$ values of 1.0-2.2 $\mu \mathrm{g} / \mathrm{mL}$ against all tested cells [54]. The endophytic fungus Phomopsis sp. isolated from the plant Cistus salvifolius, yielded four new pyrenocines, pyrenocines J-M (91-94). They exhibited antibacterial and algicidal activities against E. coli, B. megaterium, and C. fusca. The antifungal assay showed that 92 and 94 were active against M. violaceum, and compounds 91-92, and 94 were active against Septoria tritici [55]. An unusual pyrone metabolite, phomopsis-H76 C (95), was isolated from Phomopsis sp. (\#zsu-H76), which inhibited the growth of SIV branch [43]. The structures of pyrones (83-95) are shown in Figure 3.<smiles>[R]Cc1c([R])c([R])c2c(c1[R])COC2=O</smiles>

$\begin{array}{lllll} & \mathrm{R}_{1} & \mathrm{R}_{2} & \mathrm{R}_{3} & \mathrm{R}_{4} \\ 59 & \mathrm{OCH}_{3} & \mathrm{CH}_{3} & \mathrm{H} & \mathrm{COOH}\end{array}$

$\begin{array}{lllll}59 & \mathrm{OCH}_{3} & \mathrm{CH}_{3} & \mathrm{H} & \mathrm{COOH} \\ 60 & \mathrm{CH}_{3} & \mathrm{H} & \mathrm{CHO} & \mathrm{OCH}_{3}\end{array}$

$\begin{array}{lllll}61 & \mathrm{CH}_{3} & \mathrm{H} & \mathrm{CH}_{3} & \mathrm{OH}\end{array}$

$\begin{array}{lllll}62 & \mathrm{CH}_{3} & \mathrm{H} & \mathrm{CH}_{3} & \mathrm{OCH}_{3}\end{array}$

$63 \mathrm{CH}_{2} \mathrm{OH} \quad \mathrm{H} \quad \mathrm{CH}_{3} \quad \mathrm{OCH}_{3}$

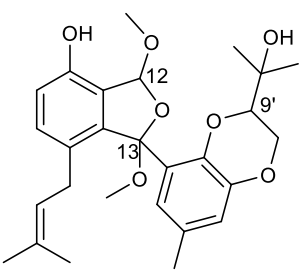

$6912 R, 13 S, 9 ' S$

$7012 S, 13 R, 9$ 'S

$7112 S, 13 S, 9$ 'S

$7212 R, 13 R, 9$ 'S

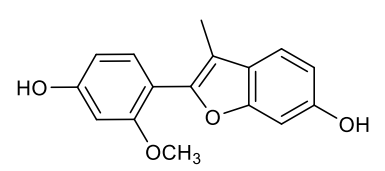

80<smiles>[R]C(C)CCCCC1OC(=O)c2cc(O)c(O)c(O)c21</smiles>

$64 \mathrm{R}=\mathrm{H}$

$65 \mathrm{R}=\mathrm{OH}$

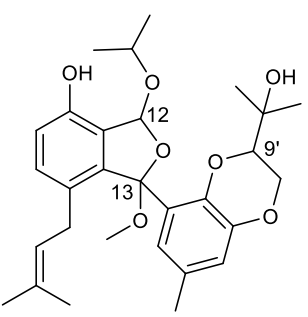

$7312 R, 13 S, 9 ' S$

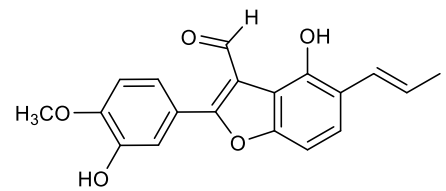

81<smiles>Cc1cc2c(c3c1C(=O)Oc1c(C)c4c(c(O)c1O3)C(=O)OC4)OCO2</smiles>

66

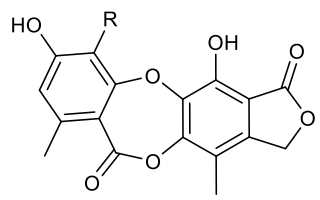

$67 \mathrm{R}=\mathrm{CH}_{2} \mathrm{OH}$ $68 \mathrm{R}=\mathrm{CHO}$<smiles>[2H]Cc1oc(=O)cc(OC)c1CO</smiles>

$83 \mathrm{R}=\mathrm{OH}$ $84 \mathrm{R}=\mathrm{H}$<smiles>CCc1c(OC)cc(=O)oc1C</smiles>

90<smiles>COc1cc(=O)oc(CO)c1[C@H](C)O</smiles>

85<smiles>[R]C([R])C[C@H]1Cc2oc(=O)cc(OC)c2CO1</smiles>

$91 \mathrm{R}_{1}=\mathrm{R}_{2}=\mathrm{H}$ $92 \mathrm{R}_{1}=$ =" $\mathrm{O}$ (keto) $\mathrm{R}_{2}=\mathrm{CH}_{3}$<smiles>COc1cc(=O)oc2c1[C@H](O)CO[C@H]2O</smiles>

86<smiles>CCCC(O)Cc1oc(=O)cc(OC)c1CO</smiles>

$93 \mathrm{R}=\mathrm{OAC}$ $94 \mathrm{R}=\mathrm{H}$<smiles>CCC(=O)Cc1c(OC)cc(=O)oc1CC</smiles><smiles>COc1cc(=O)oc(C)c1CCO</smiles><smiles>[R7][R]([H])([H])[B]</smiles>

Figure 3. Chemical structures of compounds 59-95 from Phomopsis. 


\subsubsection{Quinones}

Quinones are natural bioactive molecules with unsaturated cyclic diketones, such as cytotoxic, antimicrobial, antiviral and anti-inflammatory activities. In recent years, the development of new anti-tumor quinones and their derivatives as lead compounds has become a hot topic [110,111]. Studies of the endophytic fungus Phomopsis sp. HCCB04730 associated with stems of Radix Stephaniae Japonicae obtained six known naphthoquinones 96101. These metabolites showed cytotoxic activities against A549, MDA-MB-231 and PANC-1 cancer cells with $\mathrm{IC}_{50}$ values of $1.1-120.5 \mu \mathrm{g} / \mathrm{mL}$, and anti-HIV activities with $\mathrm{IC}_{50}$ values between 1.6-26.8 $\mu \mathrm{g} / \mathrm{mL}$ [56]. Altersolanol B (102) was separated from P. longicolla HL-2232 of leaves of Bruguiera sexangula var. rhynchopetala collected from the South China Sea. Compound 102 showed antibacterial activity against Vibrio parahaemolyticus (MIC $=2.5 \mu \mathrm{g} / \mathrm{mL}$ ) and Vibrio anguillarum (MIC $=5 \mu \mathrm{g} / \mathrm{mL}$ ) [57]. A cytotoxic anthraquinone described as altersolanol A (103), was extracted from Phomopsis sp. (PM0409092) isolated from Nyctanthes arbor-tristis. Compound $\mathbf{1 0 3}$ had cytotoxic activity to 34 human cancer cells in vitro and gave the mean $\mathrm{IC}_{50}\left(\mathrm{IC}_{70}\right)$ value of $0.005 \mu \mathrm{g} / \mathrm{mL}(0.024 \mu \mathrm{g} / \mathrm{mL})$ [58]. A new tetrahydroanthraquinone, named (2R,3S)-7-ethyl-1,2,3,4-tetrahydro-2,3,8-trihydroxy-6-methoxy3-methyl-9,10-anthracenedione (104), was separated from Phomopsis sp. PSU-MA214 associated with mangrove plant Rhizophora apiculata. Compound 104 was found to have low cytotoxic activity against MCF-7 and antibacterial activity against S. aureus ATCC25923 and methicillin-resistant Staphylococcus aureus SK1 [60]. The extraction of fungus P. foeniculi associated with Foeniculum vulgare in Bulgaria, resulted in the isolation of two octaketides anthracenones, altersolanols A (103) and J (105). They exhibited phytotoxic activities by leaf puncture bioassay [59]. Four known compounds were isolated from Phomopsis sp. derived from Notobasis syriaca, including 2-hydroxymethyl- $4 \beta, 5 \alpha, 6 \beta$-trihydroxycyclohex-2-en (106), (-)-phyllostine (107), (+)-epiepoxydon (108), and (+)-epoxydon monoacetate (109). All metabolites exhibited antifungal (M. violaceum), antibacterial (E. coli, B. megaterium), and algicidal activities (C. fusca), but 106 and 108 were inactive against $M$. violaceum [61]. A novel dihydronaphthalenone, phomonaphthalenone A (110), was derived from Phomopsis sp. HCCB04730. In terms of bioactive evaluation, compound $\mathbf{1 1 0}$ showed weak cytotoxic activity and moderate inhibitory activity on HIV with $\mathrm{IC}_{50}$ value of $11.6 \mu \mathrm{g} / \mathrm{mL}$ [56]. Ampelanol (111) was extracted from Phomopsis sp. HNY29-2B isolated from mangrove plant A. ilicifolius. Compound $\mathbf{1 1 1}$ showed antibacterial activity towards B. subtilis and S. aureus with MIC of 25 and $50 \mu \mathrm{M}$ [62]. The structures of quinones (96-111) are shown in Figure 4.

\subsubsection{Phenols}

Phenols are a kind of secondary metabolites which are widely distributed and have important physiological functions. They normally have antioxidant activity and play an important role in food industry [112]. Phomosine K (112) isolated from a Phomopsis strain showed remarkable antibacterial activity against Legionella pneumophila Corby and E. coli K12 [61]. Five known metabolites, phomosines A-D (113-116) and phomosine I (117) were isolated from a Phomopsis strain derived from Ligustrum vulgare. They had antibacterial and antifungal activities against $B$. megaterium and M. violaceum, except 116 was not active against $B$. megaterium. Moreover, compounds 113 and 116 inhibited the growth of algae [63]. Two new diphenyl ethers (118-119) were obtained from the culture of $P$. asparagi isolated from the rhizome of Paris polyphylla var. yunnanensis, collected in Kunming, Yunnan, China. These compounds displayed anti-methicillin-resistant $S$. aureus (anti-MRSA) activities with inhibition zone diameters (IZD) $10.8 \pm 2.0$ and $11.4 \pm 1.8 \mathrm{~mm}$, respectively [64]. Three new diphenyl ethers, 4-(3-methoxy-5-methylphenoxy)-2-(2-hydroxyethyl)-6-methylphenol (120), 4-(3-hydroxy-5-methylphenoxy)-2-(2-hydroxyethyl)-6-methylphenol (121), and 4-(3methoxy-5-methylphenoxy)-2-(3-hydroxypropyl)-6-methylphenol (122), were extracted from P. fukushii of Paris polyphylla var. yunnanensis. Compounds 120-122 showed antiMRSA activities and provided an IZD of $20.2 \pm 2.5 \mathrm{~mm}, 17.9 \pm 2.2 \mathrm{~mm}$, and $15.2 \pm 1.8 \mathrm{~mm}$, respectively [65]. An endophytic fungus P. fukushii, separated from the rhizome of Paris polyphylla var. yunnanensis, gave three new isopentylated diphenyl ethers (123-125). Com- 
pounds (123-125) had notable anti-MRSA activities, and their IZD were $21.8 \pm 2.4 \mathrm{~mm}, 16.8$ $\pm 2.2 \mathrm{~mm}$, and $15.6 \pm 2.0 \mathrm{~mm}$, respectively [66]. Two new diphenyl ethers (126-127) were obtained from the fermentation products of $P$. fukushii isolated from Paris polyphylla var. yunnanensis. The results of the anti-MRSA activities assay revealed that compounds $\mathbf{1 2 6}$ and 127 gave IZD of $13.8 \pm 1.5 \mathrm{~mm}$ and $14.6 \pm 1.6 \mathrm{~mm}$, respectively [67]. Three new napthalene derivatives (128-130) were separated from P. fukushii, an endophytic fungus isolated from Paris polyphylla var. yunnanensis. Compounds 128-130 showed anti-MRSA activities with MCI values of 4, 4 and $6 \mathrm{mg} / \mathrm{mL}$ [68]. From fermentation products of the fungus Phomopsis sp. associated with Paris polyphylla var. yunnanensis, two new naphthalene derivatives (131-132) were obtained. Compounds 131-132 displayed anti-MRSA activities with IZD of $14.5 \pm 1.2$ and $15.2 \pm 1.3 \mathrm{~mm}$ [69]. A culture of the marine fungus P. lithocarpus FS508 isolated from deep-sea sediment collected from Indian Ocean, obtained a new benzophenone, tenellone $\mathrm{H}$ (133). It showed cytotoxicity against HepG-2 $\left(\mathrm{IC}_{50}=16 \mu \mathrm{M}\right)$ and A549 $\left(\mathrm{IC}_{50}=17.6 \mu \mathrm{M}\right)[70]$.<smiles>COC1=CC(=O)c2c(O)c(C)c3cc(C)oc3c2C1=O</smiles>

96<smiles>COC1=CC(=O)c2c(O)c(C)c3c(c2C1=O)OC(C)C3</smiles>

97<smiles>COc1cc(O)c2c(c1O)C(=O)C1=C(CC(C)(O)CO1)C2=O</smiles>

98<smiles>COC1=CC(=O)c2c(O)c(C)c(CC(C)=O)c(O)c2C1=O</smiles>

99<smiles>COc1cc(O)c2c(c1)C(=O)C(CC(C)=O)=C(C)C2=O</smiles><smiles>CCc1c(OC)cc2c(c1O)C(=O)C1=C(C[C@H](O)[C@@](C)(O)C1)C2=O</smiles><smiles>COc1cc(O)c2c(c1)C(=O)[C@@H]1C[C@](C)(O)[C@@H](O)C[C@H]1[C@H]2O</smiles>

104<smiles>[R6]CC1=C[C@H](O)[C@H]2O[C@H]2C1=O</smiles>

$108 \mathrm{R}=\mathrm{H}$ $109 \mathrm{R}=\mathrm{Ac}$<smiles>O=C1C(CO)=C[C@@H](O)[C@H](O)[C@H]1O</smiles>

106<smiles>O=C1C=C(CO)C2OC1O2</smiles>

107<smiles>COc1cc(O)c2c(c1)[C@H](O)[C@H]1[C@H](C2=O)[C@@H](O)[C@H](O)[C@@](C)(O)[C@@H]1O</smiles>

111

Figure 4. Chemical structures of compounds 96-111 from Phomopsis.

The new metabolite, 16-acetoxycytosporone B (134), was sourced from Phomopsis sp. YM 355364 associated with Aconitum carmichaeli. In the bioassay, compound 134 had remarkable antifungal activity towards C. albicans, Hormodendrum compactum, and Trichophyton gypseum with MIC values of 32, 128, and $512 \mu \mathrm{g} / \mathrm{mL}$ [71]. Cultivation of Phomopsis sp. 0391 isolated from the stems of Paris polyphylla var. yunnanensis afforded cytosporone B (135) and dothiorelone A (136). These two compounds showed notable lipase inhibition and gave $\mathrm{IC}_{50}$ values of 115 and $275 \mu \mathrm{g} / \mathrm{mL}$ with Orlistat $\left(\mathrm{IC}_{50}=43 \mu \mathrm{g} / \mathrm{mL}\right)$ as positive control [72]. Cytosporone B (135) was extracted from the cultivation of Phomopsis sp. PSU-H188, an endophytic fungus from Hevea brasiliensis. 135 showed protective effect on 
INS-1 832/13 pancreatic $\beta$-cells $\left(\mathrm{EC}_{50}=11.08 \mu \mathrm{M}\right)$ [73]. Two diastereomeric antineoplastic tenellone derivatives identified as lithocarpinols A (137) and B (138), were isolated from $P$. lithocarpus FS508, a deep-sea derived fungus derived from a sediment collected in the Indian Ocean. During the cytotoxic assay, compounds 137-138 showed inhibitory effects against HepG-2, MCF-7, SF-268, and A549 cancer cells with IC $_{50}$ values ranging from 9.4 to $35.9 \mu \mathrm{mol} / \mathrm{L}$ [74]. Phomoindene A (139), a new indene derivative, was produced by Phomopsis sp. (No. GX7-4A) from the mangrove sediment of BeiHai, GuangXi, China. Compound 139 showed weak cytotoxicity againt KB, KBv 200, and MCF-7 cancer cells with $\mathrm{IC}_{50}$ values greater than $50 \mu \mathrm{moL} / \mathrm{mL}$ [75]. Then, 4-Hydroxybenzaldehyde (140) was extracted from a strain of Phomopsis sp. YM 355364. The antimicrobial activities of 140 provided MIC values at 256 and $128 \mu \mathrm{g} / \mathrm{mL}$ against B. subtilis and P. oryzae [26]. An investigation of the extracts from $P$. longicolla HL-2232, afforded a new biphenyl derivative, 5,5'-dimethoxybiphenyl-2,2'-diol (141). Compound 141 displayed antibacterial activity against $V$. parahaemolyticus with MIC value of $10 \mu \mathrm{g} / \mathrm{mL}$ [57]. A known phenylethyl alcohol, phomonitroester (142), was derived from Phomopsis sp. PSU-MA214, exhibiting cytotoxicity with $\mathrm{IC}_{50}$ value of $43 \mu \mathrm{g} / \mathrm{mL}$ against KB [60]. Cytosporone $\mathrm{U}$ (143) was isolated from the fermentation products of Phomopsis sp. FJBR-11. This compound displayed inhibitory effect on TMV with $\mathrm{IC}_{50}$ value of $144.6 \mu \mathrm{g} / \mathrm{mL}$ [76]. Altenusin (144) was extracted from Phomopsis sp. CAFT69, possessing a certain motility inhibitory and lytic activity against the zoospores of grapevine downy mildew pathogen $P$. viticola between $1-10 \mu \mathrm{g} / \mathrm{mL}$ [48] Cosmochlorins D (145) and E (146) produced by the endophytic fungus Phomopsis sp. N-125 of Ficus ampelas, showed significant cytotoxic activities against HL60 cells with $\mathrm{IC}_{50}$ values of 6.1 and $1.8 \mu \mathrm{M}$, and displayed growth-inhibition activities [77]. The structures of phenols (112-146) are shown in Figure 5.

\subsubsection{Oblongolides}

Oblongolides are a kind of natural active products with novel norsesquiterpene $\gamma$-lactone. At present, oblongolides are relatively less reported than other kinds of polyketides. Most of them exist in the fungi of Phomopsis, and mainly have cytotoxic activities [113]. Three new oblongolides, oblongolides Z (147) and Y (148) and 2-deoxy- $4 \alpha-$ hydroxyoblongolide X (154), were extracted from Phomopsis sp. BCC 9789 isolated from a wild banana (Musa acuminata) leaf. Compound 147 was found to have inhibitory effect on anti-herpes simplex virus type 1 (HSV-1) with $\mathrm{IC}_{50}$ value of $14 \mu \mathrm{M}$ and showed cytotoxicities with $\mathrm{IC}_{50}$ values at $26-60 \mu \mathrm{M}$ towards KB, BC, NCI-H187, and Vero cancer cells. Compound 148 was cytotoxic against $\mathrm{BC}\left(\mathrm{IC}_{50}=48 \mu \mathrm{M}\right)$ and 154 showed anti-HSV-1 activity with $\mathrm{IC}_{50}$ value of $76 \mu \mathrm{M}$ [78]. Five metabolites, oblongolides C1 (149), P1 (150), $X 1$ (151), and C (153), along with 6-hydroxyphomodiol (152), were separated from the strain Phomopsis sp. XZ-01, an endophytic fungus of Camptotheca acuminate. Compounds 149-153 displayed different degrees of selective inhibition in cytotoxicities against HepG2 and A549 [79]. The structures of oblongolides (147-154) are shown in Figure 5.

\subsubsection{Unclassified Polyketides}

Five compounds were obtained from Phomopsis sp. BCC 45011, including phomoxydiene C (155), 1893 A (156), mycoepoxydiene (157), deacetylmycoepoxydiene (158), and phomoxydiene A (159). All metabolites, except 156, showed strong antimalarial activities against $P$. falciparum $\mathrm{K} 1$ with $\mathrm{IC}_{50}$ values at $2.41-3.52 \mu \mathrm{g} / \mathrm{mL}$ and cytotoxicities against KB, MCF-7, NCI-H187, and Vero with $\mathrm{IC}_{50}$ values between $1.49-45.5 \mu \mathrm{g} / \mathrm{mL}$ [51]. Seven new polyoxygenated cyclohexenoids, phomopoxides A-G (160-166) were obtained from the fermentation products of Phomopsis sp. YE3250 isolated from Paeonia delavayi. All compounds exhibited $\alpha$-glycosidase inhibition with $\mathrm{IC}_{50}$ values from 1.47 to $3.16 \mathrm{mM}$, cytotoxic activities against Hela, MCF-7, and NCI-H460 cancer cell lines, and moderate antifungal activities against $C$. albicans, Aspergillus niger, P. oryzae, Fusarium avenaceum, and H. compactum [80]. A new geranylcyclohexenetriol, named phomentrioloxin (167), was obtained from Phomopsis sp. of the plant Carthamus lanatus. This compound showed 
phytotoxic activity and might be considered a potential mycoherbicide [81]. A new natural cyclopentenone, phomotenone (168) was produced by Phomopsis sp. Compound 168 displayed remarkable antifungal, antibacterial, and antialgal activities against $M$. violaceum, $E$. coli, B. megaterium, and C. fusca [40]. The cytotoxicity-guided investigation of the fungus Phomopsis sp. DC275 of Vitis vinifera yielded two new furanones, phomopsolidones A (170) and B (171), and a known phomopsolide B (169). All these metabolites showed weak phytotoxic and antibacterial activities [82]. The structures of unclassified polyketides (155-171) are shown in Figure 6.<smiles>[R6]c1cc(C)cc(Oc2c([R8])c([R9])c(O)c([2H])c2[R9])c1Br</smiles>

$\begin{array}{llll} & \mathrm{R}_{1} & \mathrm{R}_{2} & \mathrm{R}_{3} \\ 112 & \mathrm{CH}_{3} & \mathrm{COOCH}_{3} & \mathrm{CH}_{3} \\ 113 & \mathrm{CH}_{3} & \mathrm{COOCH}_{3} & \mathrm{CH}_{3} \\ 114 & \mathrm{CH}_{3} & \mathrm{COOCH}_{3} & \mathrm{H} \\ 115 & \mathrm{CH}_{3} & \mathrm{COOCH}_{3} & \mathrm{CH}_{3} \\ 116 & \mathrm{CH}_{3} & \mathrm{COOCH} & \mathrm{CH}_{3} \\ 117 & \mathrm{CH}_{3} & \mathrm{COOH} & \mathrm{CH}_{3} \\ 118 & \mathrm{H} & \mathrm{CH}_{2} \mathrm{OH} & \mathrm{CH}_{2} \mathrm{CH}_{2} \mathrm{OH} \\ 119 & \mathrm{H} & \mathrm{CH}_{2} \mathrm{OH} & \mathrm{CH}_{2} \mathrm{CH}_{2} \mathrm{OH} \\ 120 & \mathrm{H} & \mathrm{CH}_{3} & \mathrm{CH}_{2} \mathrm{CH}_{2} \mathrm{OH} \\ 121 & \mathrm{H} & \mathrm{CH}_{3} & \mathrm{CH}_{2} \mathrm{CH}_{2} \mathrm{OH}\end{array}$

$\mathrm{R}_{4} \quad \mathrm{R}_{5}$

$\mathrm{OH} \quad \mathrm{CH}_{3}$

$\mathrm{OH} \quad \mathrm{OHO}$

$\mathrm{OH} \quad \mathrm{CH}_{2} \mathrm{OCH}$

$\mathrm{OH} \quad \mathrm{CH}_{2} \mathrm{OCH}$

$\mathrm{CH}_{2} \mathrm{OH}$
$\mathrm{CH}$

$\mathrm{CH}_{2}$

$\mathrm{H}$

$\mathrm{H}$<smiles>CC(C)=CCOc1cc(C)cc(C(=O)C2=CCCC(O)=C2C=O)c1O</smiles>

133

$131 \mathrm{R}=\mathrm{H}$

$132 \mathrm{R}=\mathrm{OH}$
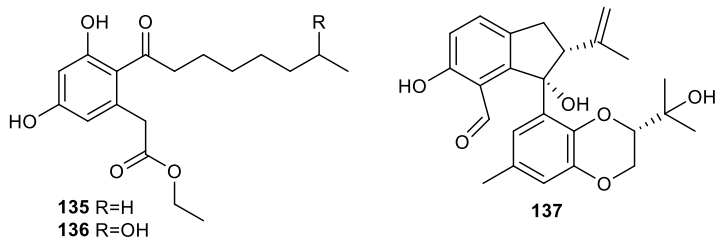

137
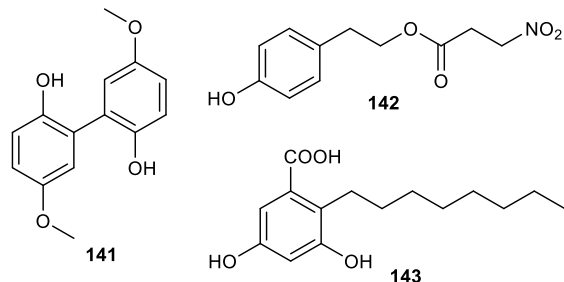

138

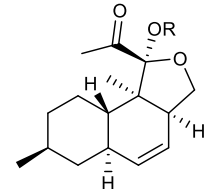

146<smiles>CCOC(=O)Cc1cc(O)cc(O)c1C(=O)CCCCCCCOC(C)=O</smiles>

134<smiles>C=C(C)C(=O)Cc1c(C)cc(Oc2cc(Br)cc(CBr)c2)cc1OC</smiles>

$\begin{array}{ll}\mathrm{R}_{1} & \mathrm{R}_{2} \\ { }_{123} & \mathrm{H}\end{array}$

$123 \mathrm{H} \quad \mathrm{OCH}_{3}$

$125 \mathrm{OH} \mathrm{OH}$<smiles>[R3]c1cc2c([R])c([R])cc(O)c2c(O)c1C(=O)CCO</smiles>

$128 \mathrm{R}_{1}=\mathrm{H} \quad \mathrm{R}_{2}=\mathrm{R}_{3}=\mathrm{OCH}_{3}$

$129 \mathrm{R}_{1}=\mathrm{H}_{2} \quad \mathrm{R}_{2}=\mathrm{OCH}_{3} \quad \mathrm{R}_{3}=\mathrm{OH}$ $130 \mathrm{R}_{1}=\mathrm{OCH}_{3} \quad \mathrm{R}_{2}=\mathrm{H} \quad \mathrm{R}_{3}=\mathrm{OCH}_{3}$<smiles>COC(=O)C1(O)/C(=C\c2ccc(O)cc2)Cc2ccc(CC=C(C)C)c(O)c21</smiles><smiles>C=C(C)[C@@H]1Cc2ccc(O)c(C=O)c2[C@@]1(O)c1cc(C)cc2c1O[C@H](C(C)(C)O)CO2</smiles><smiles>O=Cc1ccc(O)cc1</smiles>

140<smiles>COc1cc(OC)c(Cl)c(/C(C)=C/C(C)=O)c1Cl</smiles><smiles>COc1cc(OC)c(Cl)c(/C(C)=C/C(C)=C/C(C)=O)c1Cl</smiles>

$147 \mathrm{R}=\mathrm{CH}_{2} \mathrm{CH}_{2} \mathrm{Ph}$<smiles>COc1cc(O)c(C(=O)O)c(-c2cc(O)c(O)cc2C)c1</smiles>

144

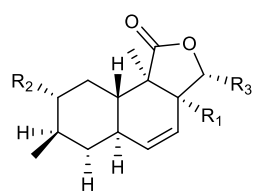

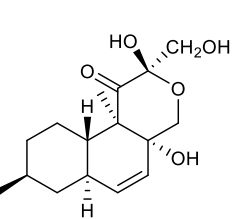

151
152

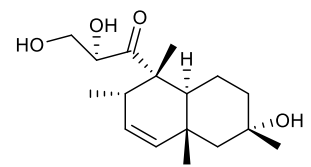<smiles>C[C@H]1CC[C@H]2C(C=C[C@@H]3[C@@H](O)O[C@H](CO)C(=O)[C@@]3(C)[C@@H]2C)C1</smiles>

$149 \quad \mathrm{R}_{1}=\mathrm{OH} \quad \mathrm{R}_{2}=\mathrm{H} \quad \mathrm{R}_{3}=\mathrm{OH}$ $150 \mathrm{R}_{1}=\mathrm{H} \quad \mathrm{R}_{2}=\mathrm{OCOCH}_{3} \quad \mathrm{R}_{3}=\mathrm{H}$ $153 \quad R_{1}=O H \quad R_{2}=H \quad R_{3}=H$

Figure 5. Chemical structures of compounds 112-154 from Phomopsis. 


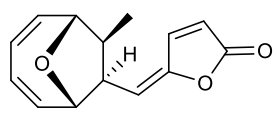

155

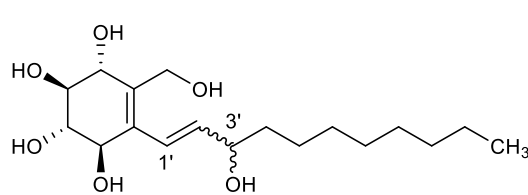

$1603^{\prime} R, \Delta^{1 '}-E$

1613 'S, $\Delta^{1}-Z$<smiles>CCCCCCCC[C@H](O)/C=C/C1=C(CO)[C@H](O)[C@@H]2O[C@H]2[C@H]1O</smiles>

$1633^{\prime} R, \Delta^{1}-E$

$1643^{\prime} S, \Delta^{1 '}-Z$<smiles>CCCCCCCCC(=O)C[C@H]1OCC2=C1[C@H](O)[C@H]1O[C@H]1[C@H]2O</smiles>

166<smiles>[R2][C@H]1C=CC(=O)O[C@@H]1[R7]</smiles>

169

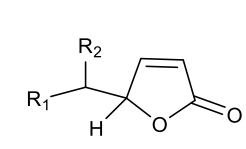

170

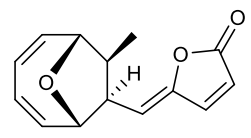

156

162<smiles>CCCCCCCC/C=C/[C@H]1OCC2=C1[C@@H](O)[C@@H]1O[C@H]1[C@H]2O</smiles>

165<smiles>C=C(C#CC1=C[C@@H](O)[C@@H](OC)[C@H](O)[C@@H]1O)CCC=C(C)C</smiles>

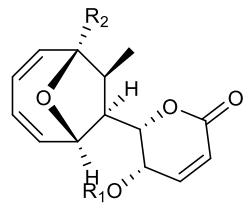

$157 \mathrm{R}_{1}=A c \mathrm{R}_{2}=\mathrm{H}$

$158 \mathrm{R}_{1}=\mathrm{R}_{2}=\mathrm{H}$

$159 \mathrm{R}_{1}=\mathrm{Ac} \mathrm{R}_{2}=\mathrm{OH}$<smiles>[R]C([R])C1CCC(=O)O1</smiles>

171

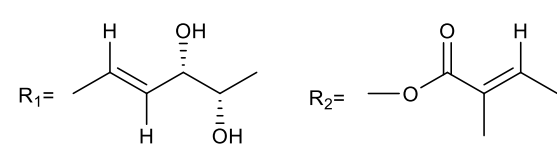

Figure 6. Chemical structures of compounds 155-171 from Phomopsis.

\subsection{Terpenoids}

Terpenoids are a kind of natural bioactive substances with isoprene as scaffold, which are widely distributed and rich in species [114,115]. Herein, a total of 38 terpenoids, including three monoterpenoids, 25 sesquiterpenoids, seven diterpenoids, and three triterpenoids, were isolated from various Phomopsis strains, accounting for $15 \%$ of all the described metabolites, second only to polyketides. It is worth noting that some terpenoids showed interesting bioactivities, such as enzyme inhibitory and anti-inflammatory activities.

\subsubsection{Monoterpenoids}

Monoterpenoids and their derivatives have a variety of biological activities, such as cytotoxic, antimicrobial, and anti-inflammatory, which have potential application value in clinical medicine [116]. Acropyrone (172) was extracted from culture of Phomopsis sp. HNY29-2B. Compound $\mathbf{1 7 2}$ showed antibacterial activity towards B. subtilis (MIC $=25 \mu \mathrm{M}$ ) and $P$. aeruginosa $(\mathrm{MIC}=50 \mu \mathrm{M}$ ) [62]. A phytotoxic pentaketide monoterpenoid, nectriapyrone (173), was produced by the fungus $P$. foeniculi [59]. According to bioassay-guided procedure, a known compound, $(1 S, 2 S, 4 S)$-trihydroxy- $p$-menthane (174) was obtained from Phomopsis sp., displaying antialgal activity against $C$. fusca and antibacterial activity against E. coli and B. megaterium [40]. The structures of monoterpenoids (172-174) are shown in Figure 7. 
<smiles>COc1cc(/C(C)=C/C(=O)O)oc(=O)c1C</smiles><smiles>C/C=C(\C)c1cc(OC)c(C)c(=O)o1</smiles><smiles>CC1=C2OCC[C@]2(C)[C@@H]2CC(C)(C)C=C1C2=O</smiles>

175<smiles>CC1=C2CC[C@@]2(C)[C@H]2C(=CC(C)(C)[C@H]2O)C1=O</smiles>

181<smiles>Cc1cc2c(cc1O)[C@H](C)[C@H](O)C[C@@H]2C(C)C</smiles>

186<smiles>CC1=C2CC[C@]2(C)[C@@]2(O)C(=CC1(C)C)C(=O)C(C)(C)[C@H]2O</smiles>

176<smiles>CC1C(=O)C2=CC(C)(C)C[C@]2(O)[C@@]2(C)C[C@H](O)C12</smiles>

182<smiles>Cc1cc2c(cc1O)[C@@H](C)CC(=O)C2C(C)C</smiles>

187<smiles>CC1=C2CC[C@]2(C)[C@H]2C[C@](C)(CO)C=C2C1=O</smiles>

177

183<smiles>Cc1cc2c(cc1O)[C@@H](C)CCC2=O</smiles>

188<smiles>CC1(C)[C@@H](O)CC[C@]2(C)[C@@H]3Cc4c(O)cc5c(c4O[C@@]3(C)CC[C@@H]12)COC5=O</smiles>

189<smiles>C/C=C(\C=C\[C@]1(O)C(C)=CC(=O)CC1(C)C)C(=O)O</smiles>

193<smiles>CC1(C)C(=O)CC[C@]2(C)[C@@H]1CC[C@]1(C)Oc3c(c(O)cc4c3COC4=O)C[C@H]12</smiles>

190<smiles>CC1=CC(=O)CC(C)(C)[C@@]1(O)/C=C/C(C)=C\C(=O)O</smiles>

194<smiles>CC(=O)c1c(C)cc(O)c2c1O[C@]1(C)CCC3[C@@H](C)C(=O)CCC[C@]3(C)[C@H]1C2</smiles>

191<smiles>Cc1ccc2c3c1C(=O)OC[C@]3(C)[C@H](O)C2(C)C</smiles>

195<smiles>CC(=O)OC1CCC2(C)[C@H](Cc3c(O)cc4c(c3O1)COC4=O)[C@@H](C)CC[C@@H](C)[C@H]2C</smiles>

192<smiles>[R]C1C[C@@H](O)[C@H]2C=C(C)[C@H](/C(C)=C/C)[C@H](/C=C/C=C/C(=O)O)[C@@H]2[C@H]1C</smiles>

$197 \mathrm{R}=\mathrm{OH}$ $198 \mathrm{R}=\mathrm{H}$<smiles>C=C(C)[C@@H]1C[C@]2(C)C(=CC1=O)CC[C@@H](OC(=O)C(C)C(O)/C=C/C=C/CC(C)O)[C@@H]2C</smiles>

199

Figure 7. Chemical structures of compounds 172-199 from Phomopsis.

\subsubsection{Sesquiterpenoids}

Sesquiterpenoids are the most abundant members of natural terpenoids because of their various structures and notable bioactivities. The chemical components of sesquiterpenoids had been found in plants, animals, microorganisms and marine organisms [117,118]. A series of sesquiterpenoids (175-184 and 195) were isolated from a strain of Phomopsis sp. TJ507A obtained from Phyllanthus glaucus. All compounds exhibited the inhibitory rates in the range of $19.4 \%$ to $43.8 \%$ against $\beta$-site amyloid precursor protein cleaving 
enzyme 1 (BACE1) at the concentration of $40 \mu \mathrm{M}$ [83]. From the endophytic fungus $P$. cassia associated with Cassia spectabilis, two new diastereoisomeric cadinanes sesquiterpenes (185-186), (7S,10R)-3-hidroxicalamen-8-one (187), and aristelegone-A (188) were isolated. Compounds 185-188 showed antifungal activities towards Cladosporium cladosporioides and Cladosporium sphaerospermum, and acetylcholinesterase inhibitory activities [84]. Four metabolites were separated from $P$. archeri of Vanilla albidia, including three new sesquiterpenes, phomoarcherins A-C (189-191), and a known kampanol A (192). The cytotoxic activites of 189-192 provided $\mathrm{IC}_{50}$ values from 0.1 to $19.6 \mu \mathrm{g} / \mathrm{mL}$ against five cholangiocarcinoma cells (KKU-100, KKU-M139, KKU-M156, KKU-M213, and KKU-M214), and 189-190 showed little activities against the $\mathrm{KB}$ with $\mathrm{IC}_{50}$ values at 42.1 and $9.4 \mu \mathrm{g} / \mathrm{mL}$. Compound 190 displayed antimalarial activity against $P$. falciparum $\left(\mathrm{IC}_{50}=0.79 \mu \mathrm{g} / \mathrm{mL}\right)$ [85]. A new sesquiterpene, (+)-S-1-methyl-abscisic-6-acid (193), and a known (+)-S-abscisic acid (194), were extracted from P. amygdali of Call midge. Compounds 193-194 showed antibacterial activities against $P$. aeruginosa 2033E with $\mathrm{MIC}$ at 30 and $58 \mu \mathrm{g} / \mathrm{mL}$ [86]. Curcumol (196), isolated from P. castaneae-mollissimae GQH87 derived from medicinal plant Artemisia annua, showed cytotoxicity against MCF-7, HepG2, and A549 with $\mathrm{IC}_{50}$ values of 25.73, 65.18, and $178.32 \mu \mathrm{g} / \mathrm{mL}$, respectively [87]. The cultivation of fungus Phomopsis sp. CAFT69, afforded two bioactive compounds, 9-hydroxyphomopsidin (197) and phomopsidin (198). Both of them showed motility inhibition and lytic activities on the zoospores of grapevine downy mildew pathogen P. viticola [48]. AA03390 (199) was isolated from a strain of P. lithocarpus FS508. The compound had low cytotoxicity with $\mathrm{IC}_{50}$ values of $25.5-29.6 \mu \mathrm{M}$ against HepG-2, MCF-7, SF-268, and A549 [70]. The structures of sesquiterpenoids (175-199) are shown in Figure 7.

\subsubsection{Diterpenoids}

Diterpenoids are a kind of terpenoids with various skeletons. They possess significant pharmacological activities, such as cytotoxic, antimicrobial, and anti-inflammatory activities [119]. A new diterpenes, libertellenone J (200), was derived from fungus Phomopsis sp. S12 isolated from Illigera rhodantha. This compound showed anti-inflammatory activity by reducing the production of NO, IL- $1 \beta$, IL- 6 and TNF- $\alpha$, and inhibiting MAPKs and NF-kB pathways [88]. Four metabolites were extracted from Phomopsis sp. S12, including three new pimaranes, libertellenone $T$ (202), pedinophyllols K (203) and L (204), together with a known compound, libertellenone C (201). Compounds 201-204 showed different degrees of anti-inflammatory activities against inhibiting the production of inflammatory factors (IL-1 $\beta$, IL-6) by lipopolysaccharide in macrophages [89]. Secondary metabolites from fungus $P$. amygdali contained two known compounds, fusicoccin J (205) and $3 \alpha-$ hydroxyfusicoccin J (206). Biologically, compounds 205-206 showed antibacterial activities against $P$. aeruginosa 2033E with MICs at $26 \mu \mathrm{g} / \mathrm{mL}$ [86]. The structures of diterpenoids (200-206) are shown in Figure 8.

\subsubsection{Triterpenoids}

Triterpenoids are a kind of organic compounds widely found in nature. They have attracted the attention of researchers because their structural diversity and rich bioactivities [120]. A new euphane triterpenoid, 3S,22R,26-trihydroxy-8,24E-euphadien-11-one (207), was isolated from P. chimonanthi obtained from medicinal plant Tamarix chinensis in the yellow river delta, Dongying. Compound 207 exhibited cytotoxicity against A549, MDA-MB-231, and PANC-1 cancer cells with $\mathrm{IC}_{50}$ values of 20.32, 19.87 and $30.45 \mu \mathrm{M}$, respectively [90]. The fungus Phomopsis sp. SNB-LAP1-7-32, occurring from plant Diospyros carbonaria, produced a first lupane-type triterpenoid, betulinic acid (208). Compound 208 displayed antiviral activity on inhibiting RNA-dependant RNA polymerase with IC $_{50}$ values of $4.3 \mu \mathrm{M}$ and cytotoxicity against HCT-116 and MRC-5 [91]. Oleanolic acid (209) was extracted from P. castaneae-mollissimae GQH87, which showed cytotoxicity against MCF-7, HepG2, and A549 with $\mathrm{IC}_{50}$ values of $16.61,39.53$, and $40.08 \mu \mathrm{g} / \mathrm{mL}$, respectively [87]. The structures of triterpenoids (207-209) are shown in Figure 8. 
<smiles>C=C[C@]1(C)C=C2C(=O)C(O)=C3[C@@](C)(C(=O)OC)CC[C@H](O)[C@]3(C)[C@@H]2CC1</smiles>

200<smiles>CC1(C)CCC[C@]2(C)C3=C(C(=O)C(O)=C12)[C@@H]1OC[C@H](O)[C@]1(C)CC3</smiles>

204<smiles>C=C[C@]1(C)C=C2C(=O)C(O)=C3[C@@](C)(CO)CC[C@H](O)[C@]3(C)[C@@H]2CC1</smiles>

201<smiles>C=C[C@]1(C)C=C2C(=O)C(O)=C3[C@@](C)(C(=O)O)CC[C@H](O)[C@]3(C)[C@@]2(C)CC1</smiles>

202<smiles>CC1(C)C(=O)CC[C@@]2(C)C3=C(CC[C@@]4(C)[C@H]3OC[C@@H]4O)C(=O)C(O)=C12</smiles>

203

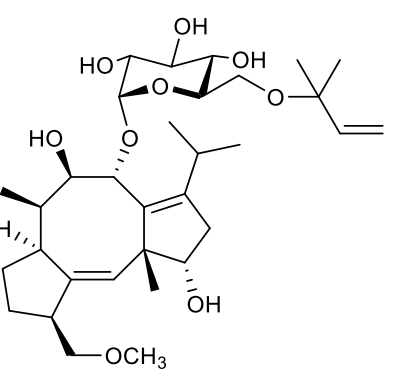

205

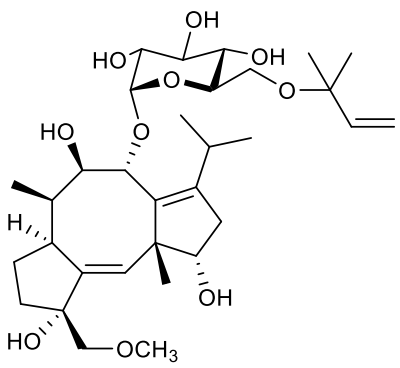

206<smiles>C/C(=C/C[C@H](O)[C@@H](C)[C@H]1CC[C@]2(C)C3=C(C(=O)C[C@]12C)[C@@]1(C)CC[C@H](O)C(C)(C)C1CC3)CO</smiles>

207

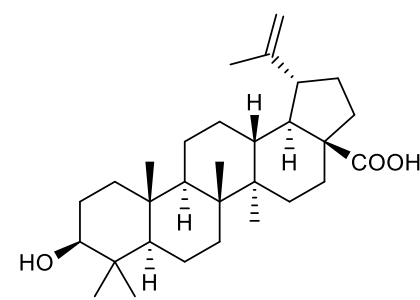

208<smiles>CC1(C)[C@@H](O)CC[C@]2(C)C3CC=C4[C@@](C)(CC[C@@]5(C(=O)O)CCC(C)(C)[C@]45C)[C@]3(C)CC[C@@H]12</smiles>

209

Figure 8. Chemical structures of compounds 200-209 from Phomopsis.

\subsection{Steroids}

Steroids are secondary metabolites with a variety of chemical structures and biological activities. At present, many researchers try to find steroidal metabolites as potential lead compounds in drug design [121]. Till now, only nine steroids were isolated from Phomopsis and showed antifungal, anti-inflammatory, and antiviral activities. Five steroids were derived from culture of Phomopsis sp., an endophytic fungus separated from A. carmichaeli, including (14 $\beta, 22 E)$-9,14-dihydroxyergosta-4,7,22-triene-3,6-dione (210), $(5 \alpha, 6 \beta, 15 \beta, 22 E)-6$ ethoxy-5,15-dihydroxyergosta-7,22-dien-3 one (211), calvasterols A (212) and B (213), and ganodermaside D (214). All isolated compounds displayed different degrees of selective antifungal activities against $C$. albicans, A. niger, P. oryzae, F. avenaceum, H. compactum, and T. gypseum with MIC values between 64-512 $\mu \mathrm{g} / \mathrm{mL}$ [92]. Dankasterone A (215) and $3 \beta, 5 \alpha, 9 \alpha$-trihydroxy-(22E,24R)-ergosta- 7,22-dien-6-one (216) were isolated from Phomopsis sp. YM 355364. Compound 215 showed anti-influenza activity against H5N1pseudovirus $\left(\mathrm{IC}_{50}=3.56 \mu \mathrm{M}\right)$. Compounds 215-216 showed antifungal activities against C. albicans, P. oryzae, H. compactum, and T. gypseum with MIC values of $64-512 \mu \mathrm{g} / \mathrm{mL}$ [71]. A new functionalized ergostane-type steroid, named phomopsterone B (217), was obtained from Phomopsis sp. TJ507A isolated from medicinal plant P. glaucus. Compound 217 showed anti-inflammatory activity by inhibiting iNOS enzyme with an $\mathrm{IC}_{50}$ value of $1.49 \mu \mathrm{M}$ [93]. Cyathisterol (218) was extracted from Phomopsis sp. YM 355364, displaying moderate antifungal activity toward P. oryzae $(\mathrm{MIC}=128 \mu \mathrm{g} / \mathrm{mL})$ [26]. The structures of steroids (210-218) are shown in Figure 9. 
<smiles>CC(C)[C@H](C)/C=C/[C@@H](C)[C@H]1CC[C@]2(O)C3=CC(=O)C4=CC(=O)CC[C@]4(C)[C@]3(C)CC[C@]12C</smiles>

210<smiles>CC(C)[C@H](C)/C=C/[C@H](C)[C@H]1CC[C@]2(O)C3=CC(=O)C4=CC(=O)CC[C@]4(C)[C@@]3(O)CC[C@]12C</smiles>

213<smiles>CC(C)[C@H](C)/C=C/[C@H](C)C1CCC2C3=CC(=O)[C@@]4(O)C[C@@H](O)CC[C@]4(C)C3(C)CC[C@]21O</smiles>

216

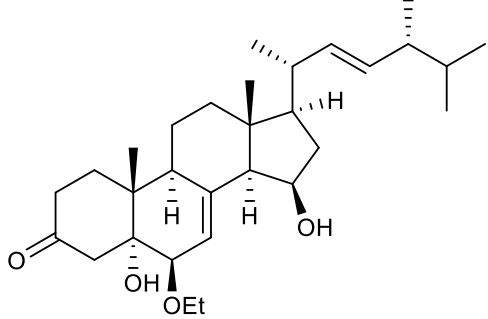

211<smiles>CC(C)[C@H](C)/C=C/[C@H](C)[C@H]1CCC2=C3C=CC4=CC(=O)CC[C@]4(C)[C@@]3(C)CC[C@]21C</smiles>

214

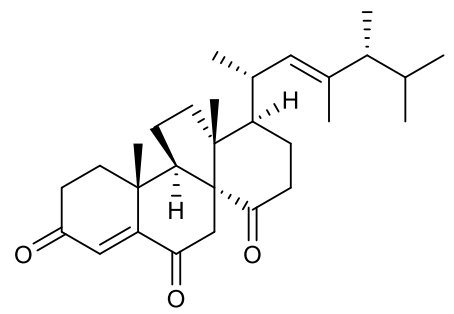

217<smiles>CC(C)[C@H](C)/C=C/[C@H](C)[C@H]1CC[C@]2(O)C3=CC(=O)C4=CC(=O)CC[C@]4(C)C3=CC[C@]12C</smiles>

212

215<smiles>CC(C)[C@H](C)/C=C/[C@H](C)[C@H]1CCC2[C@@]1(C)CCC1[C@@]3(C)CCC(=O)C=C3C=C[C@@]21O</smiles>

218

Figure 9. Chemical structures of compounds 210-218 from Phomopsis.

\subsection{Macrolides}

Macrolides are a class of medicinal compounds containing macrolactone ring structures, many of which are used as antifungal and antibacterial drugs in clinic, such as erythromycins [122]. Nowadays, a large number of macrolide antibiotics are widely used in the treatment of human diseases. Eight secondary metabolites were obtained from Phomopsis and showed cytotoxic, antimicrobial, and enzyme inhibitory activities. Three cytotoxic polyketides, Sch-642305 (219), LMA-P1 (220), and benquoine (221), were found in the endophytic fungus Phomopsis sp. CMU-LMA of Alpinia malaccensis. Compounds 219 and 221 also displayed antimicrobial activities [94]. The endophytic fungus Phomopsis sp. IFB-ZS1-S4 provided a known aspergillide C (222), which had moderate inhibitory effect on neuraminidase in vitro with $\mathrm{IC}_{50}$ value of $5.59 \mu \mathrm{M}$ [37]. Four highly oxygenated tenellone-macrolide conjugated dimers, lithocarpins A-D (223-226), were obtained from P. lithocarpus FS508 isolated from the deep-sea sediment sample collected in the Indian Ocean. All metabolites (223-226) showed cytotoxic activities against three human tumor cells (SF-268, MCF-7, and HepG-2) with $\mathrm{IC}_{50}$ values in the range of 17.0-52.2 $\mu \mathrm{M}$ [95]. The structures of macrolides (219-226) are shown in Figure 10. 
<smiles>CC1CCC[C@H]2C(=O)C=C[C@@H](O)[C@H]2CC(=O)O1</smiles>

219

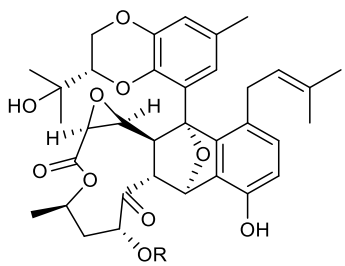

$223 \mathrm{R}=\mathrm{AC}$
$224 \mathrm{R}=\mathrm{H}$

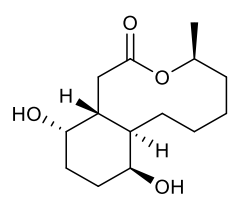

220

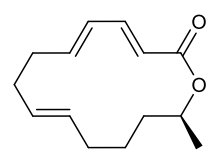

221

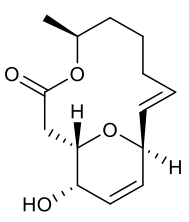

222

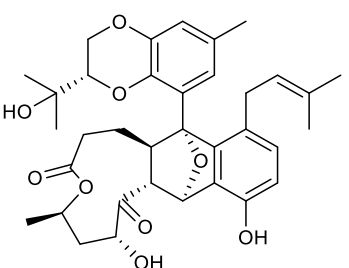

225

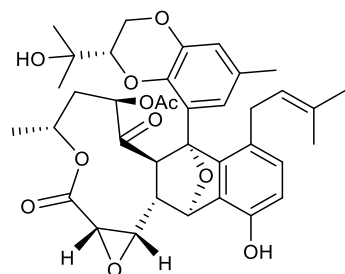

226

Figure 10. Chemical structures of compounds 219-226 from Phomopsis.

\subsection{Alkaloids}

Alkaloids are important nitrogen-containing organic compounds widely existing in microorganisms. At present, some alkaloids have been used to treat human diseases [123]. A total of 16 alkaloids have been isolated from Phomopsis and display various important bioactivities, such as cytotoxic, antibacterial, anti-inflammatory activities. Two compounds with special carbon skeleton, named phomopchalasins B (227) and C (232) were isolated from Phomopsis sp. shj2, an endophytic fungus obtained from the stems of Isodon eriocalyx var. laxiflora. Compound 232 showed cytotoxic activity against HL-60, SMMC-7721, and A-549 with $\mathrm{IC}_{50}$ values of $14.9,22.7$, and $21.1 \mu \mathrm{M}$, and displayed anti-inflammatory activity by reducing $\mathrm{NO}$ production $\left(\mathrm{IC}_{50}=11.2 \mu \mathrm{M}\right)$. In addition, compounds 227 and 232 showed antimigratory activities against MDA-MB-231 with $\mathrm{IC}_{50}$ values of 19.1 and $12.7 \mu \mathrm{M}$ [96]. Chemical investigation of Phomopsis spp. xy21 and xy22 obtained from leaves of the mangrove tree $X$. granatum, collected in Trang Province, Thailand, led to the isolation of a new cytochalasin, phomopsichalasin $G$ (228). It showed cytotoxicities against HCT-8, HCT- 8/T, A549, MDA-MB-231, and A2780 cancer cells with $\mathrm{IC}_{50}$ values between 3.4-8.6 $\mu \mathrm{M}$ [97]. Three known compounds, namely 18-metoxycytochalasin J (229), cytochalasins H (230) and J (231), were obtained from Phomopsis sp. isolated from the nut of Garcinia kola. These three compounds exhibited cytotoxicities against HeLa $\left(\mathrm{LC}_{50}=3.66-35.69 \mu \mathrm{g} / \mathrm{mL}\right)$ and Vero $\left(\mathrm{LC}_{50}=73.88-129.10 \mu \mathrm{g} / \mathrm{mL}\right)$, and different degrees of antibacterial activities against six bacterial pathogens (Vibrio cholera SG24, V. cholera CO6, V. cholera NB2, V. cholera PC2, Shigella flexneri SDINT, and S. aureus ATCC 25923) [98]. The cytochalasins, epoxycytochalasin H (234) and cytochalasin N (233) and H (230), were extracted from Phomopsis sp. By254 derived from the root of Gossypium hirsutum. They showed remarkable antifungal activities with $\mathrm{IC}_{50}$ values between $0.1-50 \mu \mathrm{g} / \mathrm{mL}$ against $S$. sclerotiorum, Bipolaris maydis, Fusarium oxysporum, B. cinerea, Bipolaris sorokiniana, Gaeumannomyces graminis var. tritici and Rhizoctonia cerealis [99]. Cytochalasins $\mathrm{H} \mathrm{(230)}$ and J (231), and alternariol (55) were extracted from Phomopsis sp. of Senna spectabilis and showed anti-inflammatory activities by inhibiting the production of reactive oxygen species (ROS). Compound 230 also showed antifungal and acetylcholinesterase enzyme (AChE) inhibitory activities [49]. Cytochalasin J (231) was derived from P. asparagi of plant Peperomia sui and exhibited antiandrogen activity $\left(\mathrm{IC}_{50}=6.2 \mu \mathrm{M}\right)$ [100]. The antibacterial diaporthalasin (235) was extracted from Phomopsis sp. PSU-H188, showing anti-MRSA activity with MIC of $4 \mu \mathrm{g} / \mathrm{mL}$ [73]. A phenylfuropyridone racemate, (+)-tersone $\mathrm{E}(\mathbf{2 3 6})$, and a known ent-citridone $\mathrm{A}(\mathbf{2 3 7})$, were separated from P. tersa FS441 derived from deep-sea sediment in the Indian Ocean. Compound 236 showed cytotoxicity with $\mathrm{IC}_{50}$ values at 32.0, 29.5, 39.5 and $33.2 \mu \mathrm{M}$ towards SF-268, MCF-7, HepG-2, and A549 cancer cells. Compounds 236-237 had antibacterial activities 
against S. aureus with MIC value of 31.2 and $31.5 \mu \mathrm{g} / \mathrm{mL}$ [101]. Two new chromenopyridine derivatives, phochrodines $\mathrm{C}$ (238) and $\mathrm{D}$ (239) with $5 \mathrm{H}$-chromeno[4,3-b]pyridine, were isolated from Phomopsis sp. 33\# associated with the bark of R. stylosa in the South China Sea. Compounds 238-239 displayed anti-inflammatory activities with $\mathrm{IC}_{50}$ values of 49 and $51 \mu \mathrm{M}$ by inhibiting nitric oxide production. Moreover, compound 239 also showed antioxidant activity with $\mathrm{IC}_{50}$ value at $34 \mu \mathrm{M}$ [102]. A novel depsipeptide, PM181110 (240), was obtained from P. glabrae of Pongamia pinnata. It showed anticancer activity towards 40 human cancer cells in vitro (mean $\mathrm{IC}_{50}=0.089 \mu \mathrm{M}$ ) and 24 human tumor xenografts ex vivo (mean $\mathrm{IC}_{50}=0.245 \mu \mathrm{M}$ ) [103]. Fusaristatin $\mathrm{A}(241)$ was separated for the first time from $P$. longicolla S1B4, showing antibacterial activity against $X$. oryzae [34]. Exumolide A (242) from the strain Phomopsis sp. (No. ZH-111) significantly promoted the growth of SIV branches and showed low cytotoxic activity against Hep-2 and HepG2 [44]. The structures of alkaloids (227-242) are shown in Figure 11.

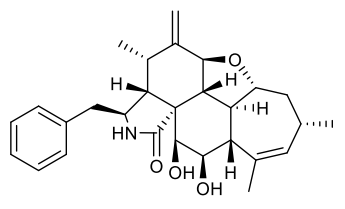

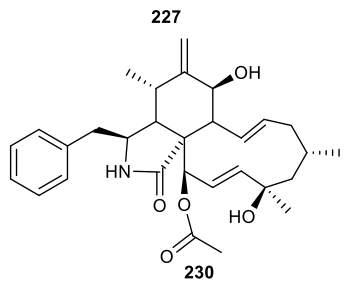

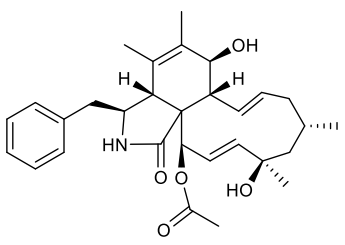

233<smiles>CC1=C[C@]2(C)Oc3[nH]cc(-c4ccccc4)c(=O)c3[C@H]2[C@H]1C</smiles>

236

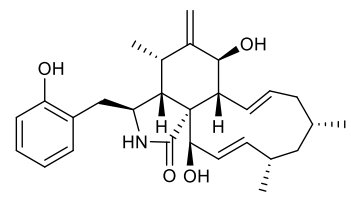

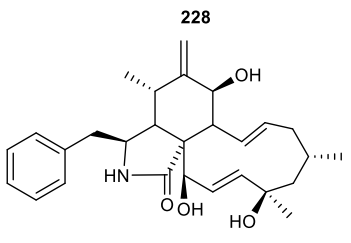

231

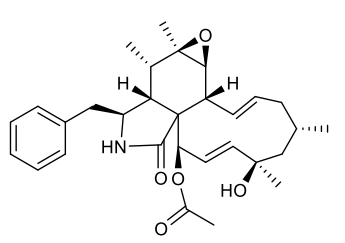
234

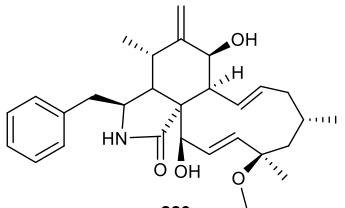

229

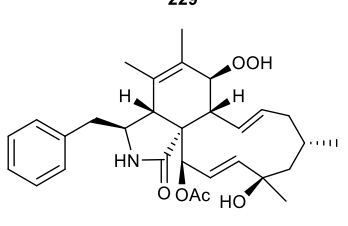

232

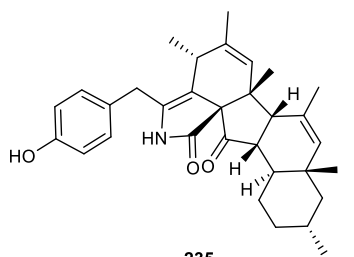

235<smiles>CCCC(CCCCCC(O)CC(=O)NC1CSCCC1C(=O)O)C(=O)NC1CCSC1</smiles>

240<smiles></smiles>

241<smiles>CC1=C[C@H](C)[C@H]2c3c(-c4ccccc4)c[nH]c(=O)c3O[C@H]2[C@H]1C</smiles>

237

$$
\begin{aligned}
& 238 \mathrm{R}=\mathrm{H} \\
& 239 \mathrm{R}=\mathrm{OH}
\end{aligned}
$$

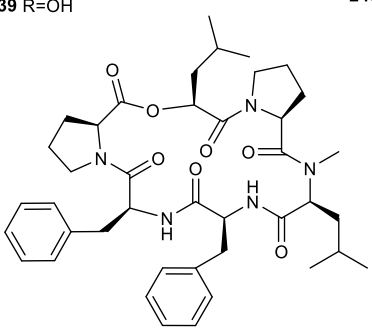

242

Figure 11. Chemical structures of compounds 227-242 from Phomopsis.

\subsection{Flavonoids}

Flavonoids are a kind of natural active substances of polyphenols. They are relatively less occurred in fungi [124]. In this review, only four flavonoids, quercetin (243) (Figure 12), luteolin (244), naringenin (245), and luteolin-7-O-glucoside (246) were isolated from $P$. castaneae-mollissimae GQH87. They displayed cytotoxic activities against MCF-7, HepG2, and $\mathrm{A} 549$ with $\mathrm{IC}_{50}$ values between 18.7 and $169.8 \mu \mathrm{g} / \mathrm{mL}$ [87]. 
<smiles>[R]c1c(-c2ccc(O)c(O)c2)oc2cc(O)cc(O)c2c1=O</smiles>

Figure 12. Chemical structures of compounds 243-246 from Phomopsis.

\section{Bioactive Secondary Metabolites from Diaporthe spp.}

In the last ten years, a total of 106 bioactive secondary metabolites have been isolated from the genus Diaporthe (Table 2). These compounds exhibit various bioactivities, such as cytotoxic, antifungal, antibacterial, antiviral, antioxidant, anti-inflammatory, phytotoxic, antitubercular, antifibrotic, antidiabetic, antimigratory, antiangiogenic, antihyperlipidemic, inhibiting leishmanicidal, activating the NF- $\mathrm{BB}$ pathway, enzyme inhibition, inhibitory effects on osteoclastogenesis, antifeedant, contact toxicity, and oviposition deterrent activities. The habitats of the Diaporthe strains were also shown in Table 2, which revealed that there are 73 (accounting for 69\%) and 32 (accounting for 30\%) compounds isolated from terrestrial and marine environments, respectively, while only one compound (1\%) was not mentioned with its habitat.

Table 2. The bioactive secondary metabolites of the genus Diaporthe during 2010-2019.

\begin{tabular}{|c|c|c|c|c|c|c|}
\hline Number & Structural Types & Compounds & Strains & Habitats $\left(T / M^{a}\right)$ & Activities & Refs. \\
\hline 247 & Xanthones & $\begin{array}{c}\text { 3,8-Dihydroxy-6- } \\
\text { methyl-9-oxo-9H- } \\
\text { xanthene-1-carboxylate }\end{array}$ & Diaporthe sp. SCSIO 41011 & Rhizophora stylosa (M) & Anti-IAV & [125] \\
\hline \multirow{2}{*}{28} & & & Diaporthe sp. GZU-1021 & Chiromanteshae- & Anti-inflammatory & [126] \\
\hline & & Phomoxanthone A & D. phaseolorum FS431 & $(\mathrm{M})$ & Cytotoxic & [127] \\
\hline 248 & \multirow[t]{11}{*}{ Chromones } & Penialidin A & Diaporthe sp. GZU-1021 & $\begin{array}{c}\text { Chiromanteshae } \\
\text { matochir (M) }\end{array}$ & Anti-inflammatory & [126] \\
\hline 35 & & (+)-Phomopsichin A & D. phaseolorum SKS019 & Acanthus ilicifolius (M) & $\begin{array}{l}\text { Inhibitory effects on } \\
\text { osteoclastogenesis }\end{array}$ & [128] \\
\hline 249 & & (-)-Phomopsichin A & D. phaseolorum SKS019 & A. ilicifolius (M) & $\begin{array}{l}\text { Inhibitory effects on } \\
\text { osteoclastogenesis }\end{array}$ & [128] \\
\hline 250 & & (+)-Phomopsichin B & D. phaseolorum SKS019 & A. ilicifolius (M) & $\begin{array}{l}\text { Inhibitory effects on } \\
\text { osteoclastogenesis }\end{array}$ & [128] \\
\hline 36 & & (-)-Phomopsichin B & $\begin{array}{l}\text { D. phaseolorum } \\
\text { SKS019Diaporthe sp. } \\
\text { GZU-1021 }\end{array}$ & $\begin{array}{l}\text { A. ilicifolius }(\mathrm{M}) \\
\text { Chiromateshaem } \\
\text { atochir }(\mathrm{M})\end{array}$ & $\begin{array}{l}\text { Inhibitory effects on } \\
\text { osteoclastogenesis } \\
\text { Anti-inflammatory }\end{array}$ & $\begin{array}{l}{[128]} \\
{[126]}\end{array}$ \\
\hline 251 & & Diaporchromanone C & D. phaseolorum SKS019 & A. ilicifolius (M) & $\begin{array}{l}\text { Inhibitory effects on } \\
\text { osteoclastogenesis }\end{array}$ & [128] \\
\hline 252 & & Diaporchromanone D & D. phaseolorum SKS019 & A. ilicifolius (M) & $\begin{array}{l}\text { Inhibitory effects on } \\
\text { osteoclastogenesis }\end{array}$ & [128] \\
\hline 40 & & Pestalotiopsone F & Diaporthe sp. SCSIO 41011 & R. stylosa (M) & Anti-IAV & [125] \\
\hline 253 & & Pestalotiopsone B & $\begin{array}{c}\text { Diaporthe sp. SCSIO } 41011 \\
\text { D. pseudomangiferaea }\end{array}$ & $\begin{array}{c}\text { R. stylosa }(\mathrm{M}) \\
\text { Tylophora ouata }(\mathrm{T})\end{array}$ & $\begin{array}{l}\text { Anti-IAV } \\
\text { Antifibrotic }\end{array}$ & $\begin{array}{l}{[125]} \\
{[129]}\end{array}$ \\
\hline 254 & & Diaportheone A & Diaporthe sp. P133 & $\begin{array}{c}\text { Pandanus } \\
\text { amaryllifolius }(\mathrm{T})\end{array}$ & Antitubercular & [130] \\
\hline 255 & & Diaportheone B & Diaporthe sp. P133 & P.amaryllifolius $(\mathrm{T})$ & Antitubercular & [130] \\
\hline 53 & \multirow[t]{3}{*}{ Chromanones } & (10S)-Diaporthin & D. terebinthifolii LGMF907 & $\begin{array}{l}\text { Schinus terebinthifolius } \\
(\mathrm{T})\end{array}$ & Antibacterial & [131] \\
\hline 256 & & Orthosporin & D. terebinthifolii LGMF907 & S. terebinthifolius (T) & $\begin{array}{l}\text { Antibacterial } \\
\text { Cytotoxic, }\end{array}$ & [131] \\
\hline 54 & & Cytosporone D & D. pseudomangiferaea & T. ouata $(\mathrm{T})$ & $\begin{array}{l}\text { Antioxidant } \\
\text { Antidiabetic }\end{array}$ & [129] \\
\hline
\end{tabular}


Table 2. Cont.

\begin{tabular}{|c|c|c|c|c|c|c|}
\hline Number & Structural Types & Compounds & Strains & Habitats (T/M $\left.{ }^{a}\right)$ & Activities & Refs. \\
\hline 257 & & $\begin{array}{l}\text { Mucorisocoumarin A } \\
\text { 3,4-Dihydro-8-hydroxy- }\end{array}$ & D. pseudomangiferaea & T. ouata $(\mathrm{T})$ & Antifibrotic & [129] \\
\hline 258 & & $\begin{array}{l}\text { 3,5-dimethyl- } \\
\text { isocoumarin }\end{array}$ & D.eres & Hedera helix $(\mathrm{T})$ & Phytotoxic & [132] \\
\hline 259 & & Diportharine A & Diaporthe sp. & Datura inoxia $(\mathrm{T})$ & Antioxidant & [133] \\
\hline 260 & \multirow[t]{5}{*}{ Furanones } & $\begin{array}{c}(1 R, 2 E, 4 S, 5 R)-1-[(2 R)-5- \\
\text { Oxotetrahydrofuran-2- } \\
\text { yl]-4,5-dihydroxy-hex- } \\
\text { 2-en-1-yl(2E)-2- } \\
\text { methylbut-2-enoate }\end{array}$ & Diaporthe sp. SXZ-19 & $\begin{array}{l}\text { Camptotheca acuminate } \\
\text { (T) }\end{array}$ & Cytotoxic & [134] \\
\hline 261 & & $\begin{array}{c}\text { Butyl 5-[(1R)-1- } \\
\text { hydroxyethyl]- } \gamma- \\
\text { oxofuran-2-butanoate }\end{array}$ & Diaporthe sp. SXZ-19 & C. acuminate $(\mathrm{T})$ & Cytotoxic & [134] \\
\hline 262 & & $\begin{array}{l}\text { 3,4-Dihydro-5'-[(1R)-1- } \\
\text { hydroxyethyl] } \\
\text { [2,2'-bifuran]-5(2H)-one }\end{array}$ & Diaporthe sp. SXZ-19 & C. acuminate $(\mathrm{T})$ & Cytotoxic & [134] \\
\hline 263 & & $\begin{array}{l}\text { 3,4-Dihydro-5'-[(1R)-1- } \\
\text { hydroxymethylethyl][2,2'- } \\
\text { bifuran]-5(2H)-one }\end{array}$ & Diaporthe sp. SXZ-19 & C. acuminate $(\mathrm{T})$ & Cytotoxic & [134] \\
\hline 264 & & Kongiidiazadione & D. Kongii & Carthamus lanatus (T) & $\begin{array}{c}\text { Phytotoxic, } \\
\text { Antibacterial }\end{array}$ & [135] \\
\hline 265 & \multirow[t]{6}{*}{ Pyrones } & Phomopsolide A & D. maritima & $\begin{array}{l}\text { Picea mariana }(\mathrm{T}) \\
\text { Picea rubens }(\mathrm{T})\end{array}$ & $\begin{array}{l}\text { Antifungal, } \\
\text { Antibiotic }\end{array}$ & [136] \\
\hline 169 & & Phomopsolide B & D. maritima & $\begin{array}{l}\text { P. mariana }(\mathrm{T}) P . \\
\text { rubens }(\mathrm{T})\end{array}$ & $\begin{array}{l}\text { Antifungal, } \\
\text { Antibiotic }\end{array}$ & [136] \\
\hline 266 & & Phomopsolide C & D. maritima & $\begin{array}{l}\text { P. mariana }(\mathrm{T}) \text { P. rubens } \\
(\mathrm{T})\end{array}$ & $\begin{array}{l}\text { Antifungal, } \\
\text { Antibiotic }\end{array}$ & [136] \\
\hline 267 & & $\begin{array}{l}(S, E)-6-(4-H y d r o x y-3- \\
\text { oxopent-1-en-1-yl)-2H- } \\
\text { pyran-2-one }\end{array}$ & D. maritima & $\begin{array}{l}\text { P. mariana }(\mathrm{T}) P . \\
\quad \text { rubens }(\mathrm{T})\end{array}$ & $\begin{array}{l}\text { Antifungal, } \\
\text { Antibiotic }\end{array}$ & [136] \\
\hline 268 & & $\begin{array}{l}\text { 7-Hydroxy-6- } \\
\text { metoxycoumarin }\end{array}$ & D. lithocarpus & $\begin{array}{c}\text { Artocarpus } \\
\text { heterophyllus }(\mathrm{T})\end{array}$ & Antifungal & [137] \\
\hline 269 & & Coumarin & D. lithocarpus & A. heterophyllus (T) & Antibacterial & [137] \\
\hline 270 & \multirow[t]{7}{*}{ Quinones } & Phyllostine acetate & D. miriciae & Cyperus iria $(\mathrm{T})$ & $\begin{array}{c}\text { Antifeedant, } \\
\text { Contact toxicity, } \\
\text { Oviposition } \\
\text { deterrent activities } \\
\text { Antifeedant, } \\
\text { Contact toxicity, } \\
\text { Oviposition } \\
\text { deterrent activities }\end{array}$ & [138] \\
\hline 271 & & Biatriosporin $\mathrm{N}$ & Diaporthe sp. GZU-1021 & $\begin{array}{l}\text { Chiromanteshae- } \\
\text { matochir }(\mathrm{M})\end{array}$ & Anti-inflammatory & [126] \\
\hline 272 & & Emodin & D. lithocarpus & A. heterophyllus (T) & $\begin{array}{c}\text { Cytotoxic, } \\
\text { Antibacterial }\end{array}$ & [137] \\
\hline 273 & & $\begin{array}{c}1,2,8- \\
\text { Trihydroxyanthraquinone }\end{array}$ & D. lithocarpus & A. heterophyllus (T) & Antibacterial & [137] \\
\hline 274 & & $(+)-2,2^{\prime}$-Epicytoskyrin A & Diaporthe sp. GNBP-10 & $\begin{array}{l}\text { Uncaria gambir Roxb } \\
\qquad(\mathrm{T})\end{array}$ & Antifungal & [139] \\
\hline 275 & & Cytoskyrin C & Diaporthe sp. & $\begin{array}{l}\text { Anoectochilus } \\
\text { roxburghii }(\mathrm{T})\end{array}$ & $\begin{array}{c}\text { Cytotoxic, } \\
\text { Activating the } \\
\text { NF-kB pathway }\end{array}$ & [140] \\
\hline 276 & & (+)-Epicytoskyrin & Diaporthe sp. & A. roxburghii $(\mathrm{T})$ & $\begin{array}{c}\text { Cytotoxic, } \\
\text { Activating the } \\
\text { NF-kB pathway }\end{array}$ & [140] \\
\hline 277 & \multirow[t]{3}{*}{ Phenols } & Tyrosol & $\begin{array}{l}\text { D. helianthin } \\
\text { D. eres }\end{array}$ & $\begin{array}{l}\text { Luehea divaricate }(\mathrm{T}) \\
\text { Vitis vinifera }(\mathrm{T})\end{array}$ & $\begin{array}{l}\text { Antagonistic } \\
\text { Phytotoxic }\end{array}$ & $\begin{array}{l}{[141]} \\
{[142]}\end{array}$ \\
\hline 278 & & $\begin{array}{c}\text { 2,5-Dihydroxybenzyl } \\
\text { alcohol }\end{array}$ & D. vochysiae LGMF1583 & Vochysia divergens $(\mathrm{T})$ & Cytotoxic & [143] \\
\hline 140 & & $\begin{array}{c}4- \\
\text { Hydroxybenzaldehyde }\end{array}$ & D. eres & V. vinifera $(\mathrm{T})$ & Phytotoxic & [142] \\
\hline
\end{tabular}


Table 2. Cont.

\begin{tabular}{|c|c|c|c|c|c|c|}
\hline Number & Structural Types & Compounds & Strains & Habitats (T/M $\left.{ }^{a}\right)$ & Activities & Refs. \\
\hline 279 & & p-Cresol & D. eres & V. vinifera $(\mathrm{T})$ & Phytotoxic & [142] \\
\hline 280 & & 4-Hydroxybenzoic acid & D. eres & $V$.vinifera $(\mathrm{T})$ & Phytotoxic & [142] \\
\hline 281 & & Arbutin & D. lithocarpus & A. heterophyllus (T) & Cytotoxic & [137] \\
\hline 113 & & Phomosine A & Diaporthe sp. F2934 & $\begin{array}{c}\text { Siparuna gesnerioides } \\
\text { (T) }\end{array}$ & Antibacterial & [144] \\
\hline 115 & & $\begin{array}{c}\text { Phomosine } C \\
\text { Flavomannin- } 6,6^{\prime} \text {-di-O- }\end{array}$ & Diaporthe sp. F2934 & S. gesnerioides $(\mathrm{T})$ & Antibacterial & [144] \\
\hline 282 & & $\begin{array}{l}\text { methyl } \\
\text { ether }\end{array}$ & D. melonis & Annona squamosal (T) & Antimicrobial & [145] \\
\hline 283 & & Acetoxydothiorelone B & D. pseudomangiferaea & T. ouata $(\mathrm{T})$ & Antifibrotic & [129] \\
\hline 284 & & Dothiorelone B & D. pseudomangiferaea & T. ouata $(\mathrm{T})$ & Antifibrotic & [129] \\
\hline 285 & & Dothiorelone L & D. pseudomangiferaea & T. ouata (T) & Antifibrotic & [129] \\
\hline 286 & & Dothiorelone G & D. pseudomangiferaea & T. ouata $(\mathrm{T})$ & Antifibrotic & [129] \\
\hline 287 & & Diaporthol A & Diaporthe sp. ECN-137 & $\begin{array}{l}\text { Phellodendron } \\
\text { amurense }(\mathrm{T})\end{array}$ & Anti-migration & [146] \\
\hline 288 & & Diaporthol B & Diaporthe sp. ECN-137 & P.amurense (T) & Anti-migration & [146] \\
\hline 289 & & Tenellone C & Diaporthe sp. SYSU-HQ3 & $\begin{array}{l}\text { Excoecaria agallocha } \\
\text { (M) }\end{array}$ & MptpB inhibitory & [147] \\
\hline 290 & & Tenellone D & Diaporthe sp. SYSU-HQ3 & E. agallocha $(\mathrm{M})$ & Anti-inflammatory & [148] \\
\hline 291 & & Diaporindene A & Diaporthe sp. SYSU-HQ3 & E. agallocha (M) & Anti-inflammatory & [148] \\
\hline 292 & & Diaporindene B & Diaporthe sp. SYSU-HQ3 & E. agallocha (M) & Anti-inflammatory & [148] \\
\hline 293 & & Diaporindene C & Diaporthe sp. SYSU-HQ3 & E. agallocha (M) & Anti-inflammatory & [148] \\
\hline 294 & & Diaporindene D & Diaporthe sp. SYSU-HQ3 & E. agallocha (M) & Anti-inflammatory & [148] \\
\hline 75 & & $\begin{array}{c}\text { Isoprenylisobenzofuran } \\
\text { A }\end{array}$ & Diaporthe sp. SYSU-HQ3 & E. agallocha $(\mathrm{M})$ & Anti-inflammatory & [148] \\
\hline 295 & Oblongolides & Oblongolide D & Diaporthe sp. SXZ-19 & C. acuminate $(\mathrm{T})$ & Cytotoxic & [134] \\
\hline 296 & & Oblongolide $\mathrm{H}$ & Diaporthe sp. SXZ-19 & C. acuminate $(\mathrm{T})$ & Cytotoxic & [134] \\
\hline 297 & & Oblongolide P & Diaporthe sp. SXZ-19 & C. acuminate $(\mathrm{T})$ & Cytotoxic & [134] \\
\hline 298 & & Oblongolide V & Diaporthe sp. SXZ-19 & C. acuminate $(\mathrm{T})$ & Cytotoxic & [134] \\
\hline 299 & $\begin{array}{l}\text { Unclassified } \\
\text { polyketides }\end{array}$ & Phomentrioloxin B & D. gulyae & C. lanatus $(\mathrm{T})$ & Phytotoxic & [149] \\
\hline 300 & & epi-Isochromophilone II & Diaporthe sp. SCSIO 41011 & R. stylosa (M) & Cytotoxic & [150] \\
\hline 301 & & Isochromophilone D & Diaporthe sp. SCSIO 41011 & R. stylosa (M) & Cytotoxic & [150] \\
\hline 302 & Monoterpenoids & $\begin{array}{c}(1 R, 2 R, 4 R) \text {-Trihydroxy- } \\
p \text {-menthane }\end{array}$ & Diaporthe sp. SXZ-19 & C. acuminate $(\mathrm{T})$ & Cytotoxic & [134] \\
\hline 303 & & Gulypyrone A & D. gulyae & C. lanatus $(\mathrm{T})$ & Phytotoxic & [149] \\
\hline 304 & & Gulypyrone B & D. gulyae & C. lanatus $(\mathrm{T})$ & Phytotoxic & [149] \\
\hline 173 & & Nectriapyrone & D. Kongii & C. lanatus $(\mathrm{T})$ & Phytotoxic & [135] \\
\hline 305 & Sesquiterpenoids & Diaporol R & Diaporthe sp. & R. stylosa (M) & Cytotoxic & [151] \\
\hline 306 & & Eremofortin F & Diaporthe sp. SNB-GSS10 & Sabicea cinerea $(\mathrm{T})$ & Cytotoxic & [152] \\
\hline 307 & & Lithocarin B & D. lithocarpus A740 & Morinda officinalis $(\mathrm{T})$ & Cytotoxic & [153] \\
\hline 308 & & Lithocarin C & D. lithocarpus A740 & M. officinalis (T) & Cytotoxic & [153] \\
\hline 309 & Triterpenoids & $\begin{array}{c}\text { 19-Nor-lanosta- } \\
5(10), 6,8,24 \text {-tetraene- } \\
1 \alpha, 3 \beta, 12 \beta, 22 S \text {-tetraol }\end{array}$ & Diaporthe sp. LG23 & Mahonia fortunei $(\mathrm{T})$ & Antibacterial & [154] \\
\hline 216 & Steriods & $\begin{array}{c}3 \beta, 5 \alpha, 9 \alpha \text {-Trihydroxy- } \\
(22 E, 24 R) \text {-ergosta-7,22- } \\
\text { dien-6-one }\end{array}$ & Diaporthe sp. LG23 & M. fortunei (T) & Antibacterial & [154] \\
\hline 310 & & Chaxine $\mathrm{C}$ & Diaporthe sp. LG23 & M. fortunei $(\mathrm{T})$ & Antibacterial & [154] \\
\hline 311 & $\begin{array}{l}\text { Ten-membered } \\
\text { lactones }\end{array}$ & Phomolide C & Diaporthe sp. & $\begin{array}{c}\text { Aucuba japonica var. } \\
\text { borealis }(\mathrm{T})\end{array}$ & $\begin{array}{l}\text { Inhibitory of } \\
\text { proliferation of } \\
\text { human colon } \\
\text { adenocarcinoma } \\
\text { cells }\end{array}$ & [155] \\
\hline 312 & & Xylarolide & D. terebinthifolii & Glycyrrhiza glabra $(\mathrm{T})$ & $\begin{array}{l}\text { Antimicrobial, } \\
\text { Cytotoxic }\end{array}$ & [156] \\
\hline 313 & & Phomolide G & D. terebinthifolii & G. glabra $(\mathrm{T})$ & Antibacterial & [156] \\
\hline 314 & & Xylarolide A & Diaporthe sp. & D. inoxia $(\mathrm{T})$ & $\begin{array}{l}\text { Cytotoxic, } \\
\text { Antioxidant }\end{array}$ & [133] \\
\hline
\end{tabular}


Table 2. Cont.

\begin{tabular}{|c|c|c|c|c|c|c|}
\hline Number & Structural Types & Compounds & Strains & Habitats (T/M $\left.{ }^{a}\right)$ & Activities & Refs. \\
\hline 315 & \multirow[t]{19}{*}{ Alkaloids } & $\begin{array}{l}\text { 18-Des-hydroxy } \\
\text { cytochalasin H }\end{array}$ & D. phaseolorum-92C & $\begin{array}{c}\text { Combretum } \\
\text { lanceolatum }(\mathrm{T})\end{array}$ & $\begin{array}{c}\text { Inhibiting } \\
\text { leishmanicidal, } \\
\text { Antioxidant, } \\
\text { Cytotoxic }\end{array}$ & [157] \\
\hline 316 & & $\begin{array}{l}\text { 21-Acetoxycytochalasin } \\
\mathrm{J}_{2}\end{array}$ & Diaporthe sp. GDG-118 & Sophora tonkinensis $(\mathrm{T})$ & $\begin{array}{l}\text { Antifungal, } \\
\text { Antibacterial }\end{array}$ & [158] \\
\hline 317 & & $\begin{array}{c}\text { 21-Acetoxycytochalasin } \\
\mathrm{J}_{3}\end{array}$ & Diaporthe sp. GDG-118 & S. tonkinensis $(\mathrm{T})$ & $\begin{array}{l}\text { Antifungal, } \\
\text { Antibacterial }\end{array}$ & [158] \\
\hline 318 & & Cytochalasin $\mathrm{J}_{3}$ & Diaporthe sp. GDG-118 & S. tonkinensis $(\mathrm{T})$ & $\begin{array}{l}\text { Antifungal, } \\
\text { Antibacterial }\end{array}$ & [158] \\
\hline 230 & & Cytochalasin $\mathrm{H}$ & $\begin{array}{l}\text { Diaporthe sp. GDG-118 } \\
\text { Diaporthe sp. GZU-1021 }\end{array}$ & $\begin{array}{l}\text { S. tonkinensis }(\mathrm{T}) \\
\text { Chiromanteshae } \\
\text { matochir }(\mathrm{M})\end{array}$ & $\begin{array}{c}\text { Antifungal, } \\
\text { AntibacterialAnti- } \\
\text { inflammatory }\end{array}$ & $\begin{array}{l}{[158]} \\
{[126]}\end{array}$ \\
\hline 319 & & $\begin{array}{c}\text { 7-Acetoxycytochalasin } \\
\text { H }\end{array}$ & Diaporthe sp. GDG-118 & S. tonkinensis $(\mathrm{T})$ & $\begin{array}{c}\text { Antifungal, } \\
\text { Antibacterial }\end{array}$ & [158] \\
\hline 231 & & Cytochalasin J & Diaporthe sp. GDG-118 & S. tonkinensis $(\mathrm{T})$ & $\begin{array}{l}\text { Antifungal, } \\
\text { Antibacterial }\end{array}$ & [158] \\
\hline 320 & & Cytochalasin E & Diaporthe sp. GDG-118 & S. tonkinensis $(\mathrm{T})$ & $\begin{array}{l}\text { Antifungal, } \\
\text { Antibacterial }\end{array}$ & [158] \\
\hline 321 & & $\begin{array}{l}\text { 21-O-Deacetyl-L- } \\
696,474\end{array}$ & Diaporthe sp. GZU-1021 & $\begin{array}{l}\text { Chiromanteshae } \\
\text { matochir }(\mathrm{M})\end{array}$ & Anti-inflammatory & [126] \\
\hline 322 & & Cordysinin A & D. arecae & Kandelia obovate (M) & Anti-angiogenic & [159] \\
\hline 323 & & 5-Deoxybostrycoidin & D. phaseolorum SKS019 & A. ilicifolius (M) & Cytotoxic & [160] \\
\hline 241 & & Fusaristatin A & D. phaseolorum SKS019 & A. ilicifolius (M) & Cytotoxic & [160] \\
\hline 324 & & Vochysiamide B & D. vochysiae LGMF1583 & $V$. divergens $(\mathrm{T})$ & $\begin{array}{c}\text { Antibacterial, } \\
\text { Cytotoxic }\end{array}$ & [143] \\
\hline 325 & & Diaporisoindole A & Diaporthe sp. SYSU-HQ3 & E. agallocha (M) & Anti-inflammatory & [148] \\
\hline 326 & & Diaporisoindole B & Diaporthe sp. SYSU-HQ3 & E. agallocha (M) & Anti-inflammatory & [148] \\
\hline 327 & & Diaporisoindole D & Diaporthe sp. SYSU-HQ3 & E. agallocha (M) & Anti-inflammatory & [148] \\
\hline & & 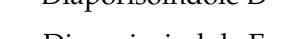 & Diaporthe sp. SYSU-HQ3 & E. agallocha $(\mathrm{M})$ & MptpB inhibitory & [147] \\
\hline 328 & & Diaporisoindole E & Diaporthe sp. SYSU-HQ3 & E. agallocha (M) & Anti-inflammatory & [148] \\
\hline 329 & & Phomopsin F & D. toxica & $-\mathrm{b}$ & Cytotoxic & [161] \\
\hline 330 & \multirow[t]{6}{*}{ Fatty acids } & $\begin{array}{l}\text { 3-Hydroxypropionic } \\
\text { acid }\end{array}$ & D. phaseolorum & $\begin{array}{l}\text { Laguncularia racemose } \\
\text { (M) }\end{array}$ & Antimicrobial & [162] \\
\hline 331 & & 3-Nitropropionic acid & D. gulyae & C. lanatus $(\mathrm{T})$ & Phytotoxic & [149] \\
\hline 332 & & Diapolic acid A & D. terebinthifolii & G. glabra (T) & Antibacterial & [156] \\
\hline 333 & & Diapolic acid B & D. terebinthifolii & G. glabra $(\mathrm{T})$ & Antibacterial & [156] \\
\hline 334 & & Diaporthsin E & Diaporthe sp. JC-J7 & Dendrobium nobile $(\mathrm{T})$ & Antihyperlipidemic & [163] \\
\hline 335 & & $\begin{array}{l}\text { 3-Hydroxy-5- } \\
\text { methoxyhex-5-ene-2,4- } \\
\text { dione }\end{array}$ & Diaporthe sp. ED2 & $\begin{array}{l}\text { Orthosiphon stamieus } \\
\text { (T) }\end{array}$ & Antifungal & [164] \\
\hline
\end{tabular}

${ }^{\mathrm{a}} \mathrm{T}$ : terrestrial environment; M: marine environment; ${ }^{\mathrm{b}}$ The habitat was not mentioned.

\subsection{Polyketides}

There are 67 polyketides reviewed from Diaporthe and they exhibit rich biological activities. Here, we classify these polyketides into the following structural types: xanthones, chromones, chromanones, furanones, pyrones, quinones, phenols, oblongolides, and unclassified polyketides.

\subsubsection{Xanthones}

Chemical investigation of Diaporthe sp. SCSIO 41011 derived from mangrove plant R. stylosa led to identification of a known compound, 3,8-dihydroxy-6-methyl-9-oxo-9Hxanthene-1-carboxylate (247) (Figure 13). It showed influenza A virus (IAV) inhibition against A/Puerto Rico/8/34 H274Y (H1N1), A/FM-1/1/47 (H1N1), and A/Aichi/2/68 (H3N2) with $\mathrm{IC}_{50}$ values of $9.40,4.80$, and $5.12 \mu \mathrm{M}$, respectively [125]. Phomoxanthone A (28) with novel carbon skeleton was isolated from the fungus Diaporthe sp. GZU-1021 derived from a red-clawed crab Chiromanteshaematochir and D. phaseolorum FS431 of deepsea sediment from the Indian Ocean. This compound showed anti-inflammatory activity by inhibiting nitric oxide (NO) production in RAW 264.7 cells with an $\mathrm{IC}_{50}$ value of $6.1 \mu \mathrm{M}$ [126], and it displayed good cytotoxicity against MCF-7, HepG-2, and A549 with $\mathrm{IC}_{50}$ values of $2.60,2.55$, and $4.64 \mu \mathrm{M}$, respectively [127]. 
<smiles>COC(=O)c1cc(O)cc2oc3cc(C)cc(O)c3c(=O)c12</smiles>

247<smiles>COC(=O)c1cc(O)cc2c1C(=O)[C@H]([C@H](O)CC(C)=O)CO2</smiles>
$252 \mathrm{R}=\mathrm{CH}_{3} .3 \mathrm{~S}$<smiles>CC(O)Cc1cc2cc(O)cc(O)c2c(=O)o1</smiles>

256<smiles>C/C=C(\C)C(=O)OC[C@H](/C=C/C(O)[C@H](C)O)[C@H]1CCC(=O)O1</smiles>

260<smiles>CC=C(C)C(=O)OC1C=CC(=O)OC1/C=C/C(=O)C(C)O</smiles><smiles>CC(C)(O)OCc1c(CO)oc2c(C(=O)O)c(O)c(O)cc2c1=O</smiles><smiles>CCCCCCCCc1cc(=O)c2c(CC(=O)OCC)cc(O)cc2o1</smiles>

253<smiles>COc1cc(O)c2c(=O)oc(C[C@H](O)CC(C)O)cc2c1</smiles>

257<smiles>Cc1ccc(O)c2c1CC(C)OC2=O</smiles><smiles>CC(O)c1ccc(C(=O)CCC(=O)O)o1</smiles>

261<smiles>C/C=C(\C)C(=O)OC1C=CC(=O)OC1/C=C/C(=O)C(C)O</smiles><smiles>COC(=O)c1cc(OC)c(O)c2oc3c(c(=O)c12)C(OC)OC(C)C3</smiles><smiles>O=c1c2c(oc3cccc(O)c13)CCC2O</smiles>

254

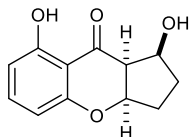

255<smiles>CCC/C=C/C1OC(=O)c2cc(O)cc(O)c2[C@H]1[18OH]</smiles><smiles>[R]C(C)c1ccc([C@H]2CCC(=O)O2)o1</smiles><smiles>N=NC1C=C(CO)C(=O)C1=O</smiles>

$262 \mathrm{R}=\mathrm{OH}$ $263 \mathrm{R}=\mathrm{OCH}_{3}$<smiles>CC(O)C(=O)/C=C/c1cccc(=O)o1</smiles><smiles>COc1cc2ccc(=O)oc2cc1Br</smiles>

$268 \mathrm{R}_{1}=\mathrm{OH} \mathrm{R}_{2}=\mathrm{OCH}_{3}$ $269 \mathrm{R}_{1}=\mathrm{H} \quad \mathrm{R}_{2}=\mathrm{H}$

Figure 13. Chemical structures of compounds 247-269 from Diaporthe.

\subsubsection{Chromones}

Chemical analysis of Diaporthe sp. GZU-1021 associated with Chiromanteshaematochir resulted in the identification of penialidin A (248) and (-)-phomopsichin B (36). They showed inhibitory effects on $\mathrm{NO}$ production with $\mathrm{IC}_{50}$ values at 11.9 and $16.5 \mu \mathrm{M}$ [126]. Six bioactive metabolites were separated from $D$. phaseolorum SKS019 derived from mangrove plant $A$. ilicifolius, including four new compounds, (-)-phomopsichin A (249), (+)phomopsichin B (250), diaporchromanones C (251) and D (252), along with two known compounds, (+)-phomopsichin A (35) and (-)-phomopsichin B (36). These metabolites showed moderate inhibition on osteoclastogenesis by inhibiting RANKL-induced NF- $\mathrm{B}$ activation [128]. Pestalotiopsones F (40) and B (253) were isolated from Diaporthe sp. SCSIO 41011. The two compounds exhibited remarkable anti-IAV activities with $\mathrm{IC}_{50}$ values between 2.52-39.97 $\mu \mathrm{M}$ [125]. Two new benzopyranones, diaportheones A (254) and B (255), were extracted from Diaporthe sp. P133 derived from Pandanus amaryllifolius. They showed moderate antitubercular activities and provided MIC values of 100.9 and $3.5 \mu \mathrm{M}$ against Mycobacterium tuberculosis $\mathrm{H}_{37} \mathrm{Rv}$ with Rifampin ( $\left.\mathrm{MIC}=0.25 \mu \mathrm{M}\right)$ as the positive control [130]. The structures of chromones (248-255) are shown in Figure 13.

\subsubsection{Chromanones}

Two isocoumarins, (10S)-diaporthin (53) and orthosporin (256), were extracted from D. terebinthifolii LGMF907 isolated from Schinus terebinthifolius. They showed antibacterial activities against the methicillin-sensitive Staphylococcus aureus (MSSA) and methicillinresistant S. aureus (MRSA) [131]. Cytosporone D (54) and mucorisocoumarin A (257) were isolated from the endophytic fungus D. pseudomangiferaea of Tylophora ouata. Compound 257 displayed anti-fibrosis activity with the inhibitory rate of $52.1 \%$ on the activation of human lung fibroblasts MRC-5 cells induced by TFG- $\beta$ at $10 \mu \mathrm{M}$. Cytosporone D (54) showed cytotoxicity toward BGC-823 $\left(\mathrm{IC}_{50}=8.1 \mu \mathrm{M}\right)$, antioxidant activity with the inhibition rate of 
$63.3 \%$ by releasing MOA at the concentration of $10 \mu \mathrm{M}$, and moderate antidiabetic activity against protein tyrosine phosphatase 1B (PTP1B) [129]. The fungus D. eres derived from pathogen-infected leaf of Hedera helix produced an isocoumarin, 3,4-dihydro-8-hydroxy-3,5dimethylisocoumarin (258), showing phytotoxic activity in Lemna paucicostata growth [132]. A novel metabolite, diportharine A (259), was obtained from the culture of Diaporthe sp. isolated from Datura inoxia. It showed notable antioxidant activity through DPPH radical scavenging effects $\left(\mathrm{EC}_{50}=10.3 \mu \mathrm{M}\right)$ [133]. The structures of chromanones (256-259) are shown in Figure 13.

\subsubsection{Furanones}

Furanones are widely used in the field of synthesis, and the synthesized products have important pharmacological activities, such as antiviral, antitumor and antimicrobial [165]. Four bioactive furanones were derived from Diaporthe sp. SXZ-19 of C. acuminate, including the new $(1 R, 2 E, 4 S, 5 R)-1-[(2 R)-5$-oxotetrahydrofuran-2-yl]-4,5-dihydroxy-hex2-en-1-yl(2E)-2-methylbut-2-enoate (260) and three linear furanopolyketides (261-263). These compounds had weak cytotoxicities against HCT 116 cells with the concentration at $10 \mu \mathrm{M}$ [134]. A new 3-substituted-5-diazenylcyclopentendione, named kongiidiazadione (264), was separated from D. kongii of plant C. lanatus, which was phytotoxic component and showed low antibacterial activity against Bacillus amyloliquefaciens [135]. The structures of furanones (260-264) are shown in Figure 13.

\subsubsection{Pyrones}

Four secondary metabolites were isolated from D. maritima of healthy Picea mariana and Picea rubens needles collected from the Acadian forest of Eastern Canada, including three dihydropyrones, phomopsolides A (265), B (169), and C (266), and a stable $\alpha$-pyrone, $(S, E)-6$ (4-hydroxy-3-oxopent-1-en-1-yl)-2H-pyran-2-one (267). All compounds showed antifungal and antibiotic activities against $M$. violaceum, Saccharomyces cerevisiae, and B. subtilis [136]. Two known metabolites, 7-hydroxy-6-metoxycoumarin (268) and coumarin (269), were isolated from the endophytic fungus D. lithocarpus obtained from Artocarpus heterophyllus. Compounds 268 showed significant antifungal activity against Sporobolomyces salminocolor with the of $12.2 \pm 0.3 \mathrm{~mm}$, and 269 had a diameter inhibition zone of $12.3 \pm 0.3 \mathrm{~mm}$ against the bacteria B. subtilis [137]. The structures of pyrones (265-269) are shown in Figure 13.

\subsubsection{Quinones}

Two cyclohexeneoxidedione derivatives, phyllostine acetate (270) and phyllostine (107), showing strong antifeedant activities on Plutella xylostella, were extracted from culture of D. miriciae of plant Cyperus iria. Compounds $\mathbf{2 7 0}$ and $\mathbf{1 0 7}$ had the feeding inhibition of $100 \%$ at $50 \mu \mathrm{g} / \mathrm{cm}^{2}$ and the $50 \%$ feeding deterrence $\left(D_{50}\right)$ values of 9 and $4.7 \mu \mathrm{g} / \mathrm{cm}^{2}$, displayed contact toxicities with the median lethal concentration $\left(\mathrm{LC}_{50}\right)$ values of 4.38 and $6.54 \mu \mathrm{g} /$ larva, and exhibited oviposition deterrent activities with the indexes of $100 \%$ and $28.6 \%$ at $50 \mu \mathrm{g} / \mathrm{cm}^{2}$, respectively [138]. The new biatriosporin $\mathrm{N}(\mathbf{2 7 1})$ was isolated from the marine-derived fungus Diaporthe sp. GZU-1021 and displayed anti-inflammatory activity by inhibiting NO production in RAW 264.7 cells with an $\mathrm{IC}_{50}$ value of $11.5 \mu \mathrm{M}$ [126]. Two anthraquinone derivatives, emodin (272) and 1,2,8-trihydroxyanthraquinone (273), were isolated from an endophytic fungus D. lithocarpus. Emodin (272) exhibited notable cytotoxic activity against murine leukemia P-388 cells $\left(\mathrm{IC}_{50}=0.41 \mu \mathrm{g} / \mathrm{mL}\right)$ and antibacterial activity against B. subtilis, M. luteus, Pseudomonas fluorescences, E. coli, and S. cerevisiae with the diameter of inhibition zones of 14.7, 13.2, 13.7, 12.7, and $11.7 \mathrm{~mm}$, respectively. Compound 273 also displayed antibacterial activity against B. subtilis, E. coli, and S. cerevisiae at 14.2, 11.3, and $10.7 \mathrm{~mm}$, respectively [137]. A bis-anthraquinone derivative, named (+)-2,2' epicytoskyrin A (274), was isolated from Diaporthe sp. GNBP-10 of Uncaria gambir Roxb. It showed antifungal activity against 22 yeast strains and 3 filamentous fungi with MICs between 16-128 $\mathrm{gg} / \mathrm{mL}$ [139]. Two cytoskyrin type bisanthraquinones, cytoskyrin C (275) and (+)-epicytoskyrin (276), were isolated from Diaporthe sp., an endophytic fungus 
obtained from Anoectochilus roxburghii. Compounds 275-276 could activate NF- $\mathrm{B}$ pathway and increase the relative activity of luciferase at the concentration of $50 \mu \mathrm{M}$, and showed cytotoxicities against SMMC-7721 cells in dose-dependent manner [140]. The structures of quinones (270-276) are shown in Figure 14.<smiles>CC(=O)OCC1=CC(=O)C2OC1C2=O</smiles>

270

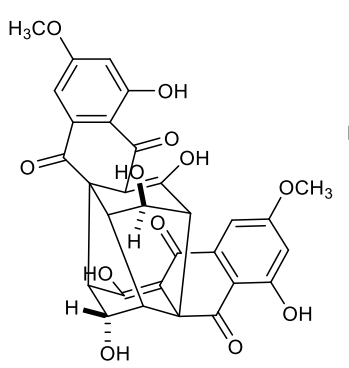

274<smiles>CC(O)c1occ2c1C(=O)c1cc(O)cc(O)c1C2=O</smiles>

271

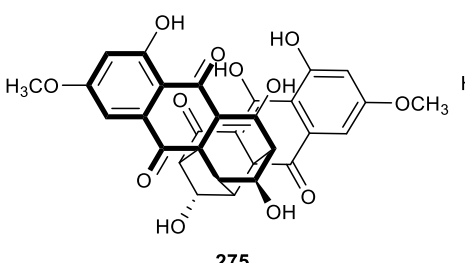

275

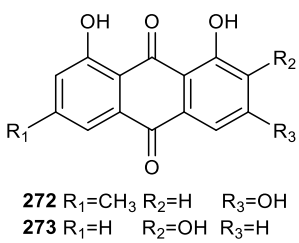

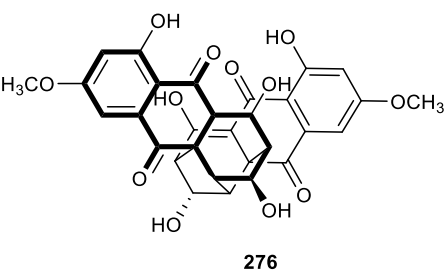

Figure 14. Chemical structures of compounds 270-276 from Diaporthe.

\subsubsection{Phenols}

The phenolic metabolite, tyrosol (277), was extracted from D. helianthi isolated from Luehea divaricate. Tyrosol showed significant antagonistic activity against the tested pathogenic bacteria (Enterococcus hirae, E. coli, M. luteus, Salmonella typhi, S. aureus, and Xanthomonas asc. Phaseoli) [141]. 2,5-Dihydroxybenzyl alcohol (278) was derived from D. vochysiae LGMF1583 of medicinal plant Vochysia divergens, which showed cytotoxic activity against A549 $\left(\mathrm{EC}_{50}=54.8 \mu \mathrm{M}\right)$ and PC3 $\left(\mathrm{EC}_{50}=9.45 \mu \mathrm{M}\right)$ [143]. Four phytotoxic compounds, 4-hydroxybenzaldehyde (140), p-cresol (279), 4-hydroxybenzoic acid (280), and tyrosol (277), were isolated from $D$. eres of grapevine ( $V$. vinifera) wood. In the leaf disk and leaf absorption bioassay, phytotoxicities of all compounds increased with the concentration ranging in 0.1-1 mg/mL [142]. Arbutin (281), obtained from an endophytic fungus $D$. lithocarpus, had moderate cytotoxicity against murine leukemia P-388 cells and gave an $\mathrm{IC}_{50}$ value at $2.91 \mu \mathrm{g} / \mathrm{mL}$ [137]. Two antibacterial metabolites, phomosines A (113) and C (115), were extracted from Diaporthe sp. F2934 of plant Siparuna gesnerioides. They were active against S. aureus, M. luteus, Streptococcus oralis, Enterococcus fecalis, Enterococcus cloacae, and Bordetella bronchiseptica with inhibition zone diameter from $6 \pm 0.62$ to $12 \pm 1.18 \mathrm{~mm}$ at the concentration of $4 \mu \mathrm{g} / \mu \mathrm{L}$ [144]. Flavomannin-6,6'-di-O-methyl ether (282) was extracted from an endophytic strain of D. melonis from Annona squamosal, which showed antimicrobial activity against S. aureus 25697, S. aureus 29213, and Streptococcus pneumoniae ATCC 49619 with MIC values of 32, 32, and $2 \mu \mathrm{g} / \mathrm{mL}$, respectively [145]. Four secondary metabolites, acetoxydothiorelone B (283), and dothiorelones B (284), L (285) and G (286), were isolated from D. pseudomangiferaea. All of them displayed antifibrotic activities with the inhibitory rates of $17.4,62.9,59.2$ and $41.1 \%$ on the activation of human lung fibroblasts MRC- 5 cells induced by TFG- $\beta$ at $10 \mu \mathrm{M}$, with pirfenidone $(53.2 \%)$ as positive control at 1 $\mathrm{mM}$ [129]. Two diphenyl ether derivatives, diaporthols A (287) and B (288), were extracted from Diaporthe sp. ECN-137 isolated from the leaves of Phellodendron amurense. Compounds 287-288 exhibited anti-migration effects on TGF- $\beta 1$-elicited MDA-MB-231 breast cancer cells with an concentration at $20 \mu \mathrm{M}$ [146]. Tenellone C (289) was obtained from Diaporthe sp. SYSU-HQ3 of mangrove plant E. agallocha, displaying inhibitory effect on $M$. tuberculosis protein tyrosine phosphatase $\mathrm{B}(\mathrm{MptpB})\left(\mathrm{IC}_{50}=5.2 \mu \mathrm{M}\right)$ [147]. Six compounds were isolated from endophytic fungus Diaporthe sp. SYSU-HQ3 derived from the branches of E. agallocha, including a new benzophenone derivative, tenellone D (290), four special 
2,3-dihydro- $1 H$-indene isomers, diaporindenes A-D (291-294), and isoprenylisobenzofuran A (75). All isolated compounds showed anti-inflammatory activities by LPS-Induced NO production in RAW 264.7 cells with $\mathrm{IC}_{50}$ values of $4.2-18.6 \mu \mathrm{M}$ [148]. The structures of phenols (277-294) are shown in Figure 15.
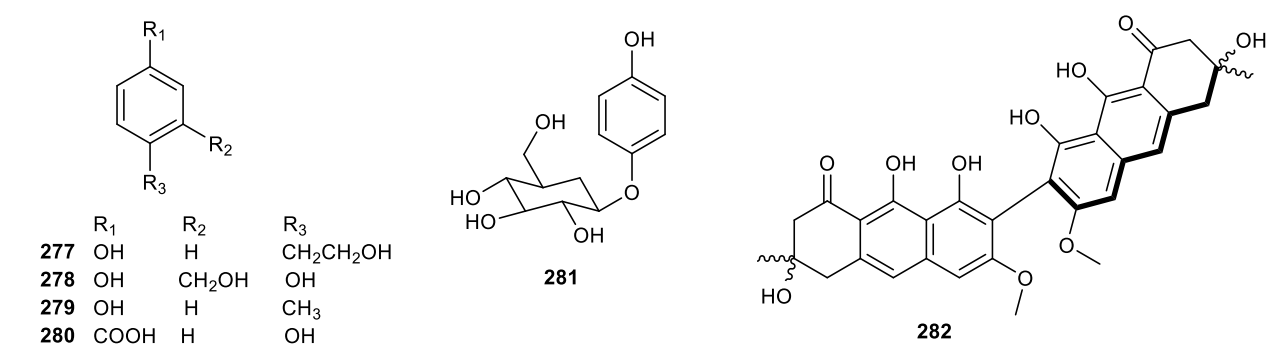<smiles>CCOC(=O)Cc1cc(O)cc(O)c1C(=O)CCCCC(CC)OC(C)=O</smiles><smiles>CCOC(=O)Cc1cc(O)cc(O)c1C(=O)CCCCC(O)CC</smiles><smiles>CCOC(=O)Cc1cc(O)cc(O)c1C(=O)CCCCC(=O)CC</smiles><smiles>CCCCCCCC(=O)c1c(O)cc(O)cc1CC(=O)OCC</smiles>

286<smiles>CC(C)=CCc1ccc(O)c(C(=O)O)c1C(=O)c1cc(C)cc2c1OC[C@H](C(C)(C)O)N2</smiles>

289<smiles></smiles>

292<smiles>Cc1cc2c(O)c(c1)OC(=O)c1c(ccc3c1OC(C)(C)C=C3)OC2</smiles>

287<smiles>COC(=O)c1c(O)ccc(CC=C(C)C)c1C(=O)c1cc(C)cc2c1OC[C@H](C(C)(C)O)CO2</smiles>

290

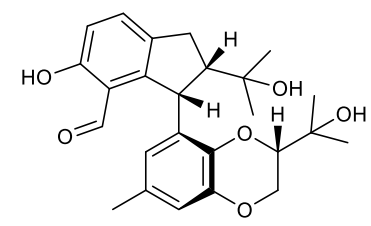

293

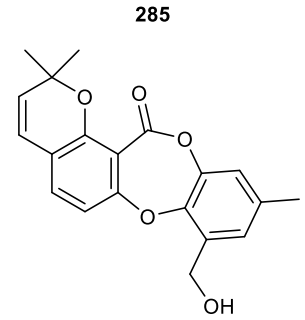

288

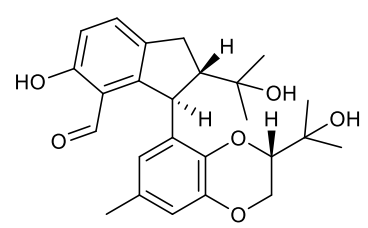

291

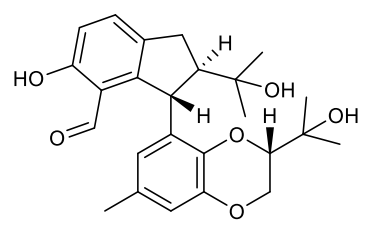

294

Figure 15. Chemical structures of compounds 277-294 from Diaporthe.

\subsubsection{Oblongolides}

Four lovastatin analogues, oblongolides D (295), H (296), P (297) and V (298), were obtained from the endophytic fungus Diaporthe sp. SXZ-19. These metabolites showed weak cytotoxic activities against HCT 116 cells with the concentration of $10 \mu \mathrm{M}$ [134]. The structures of oblongolides (295-298) are shown in Figure 16. 
<smiles>C[C@]1(O)CC[C@H]2C(=O)OC[C@H]3C=C[C@H](C1)[C@@H]32</smiles>

295<smiles>C=C(C#CC1=C[C@@H](O)[C@H](OC)[C@H](O)C1=O)CC=C(C)C</smiles>

299<smiles>C[C@H]1C[C@@H]2C[C@@H]3[C@@H](C)C[C@H]2C=C[C@@H]2COC(=O)[C@@H]2[C@@H]3C[C@@H]1O</smiles>

296<smiles>CC1C[C@H]2C[C@@H]3C(C)C[C@H]2C=C[C@H]2COC(=O)[C@@H]2[C@@H]3C[C@@H]1O</smiles>

297<smiles>CC1CC[C@]23C(=O)[C@](C)(O)OC[C@H]2C=C[C@H]3[C@H]1O</smiles>

298<smiles>CC[C@H](C)/C=C(C)/C=C/C1=CC2=C(Cl)C(=O)[C@](C)(O)[C@H](CC(C)=O)C2=CO1</smiles><smiles>CCC(C)/C=C(C)/C=C/C1=CC2=C(Cl)C(=O)[C@@]3(C)O[C@](C)(OC)[C@@H](C(=O)OC)[C@H]3[C@H]2CO1</smiles>

Figure 16. Chemical structures of compounds 295-301 from Diaporthe.

\subsubsection{Unclassified Polyketides}

Phomentrioloxin B (299) was obtained from a strain of D. gulyae isolated from C. lanatus, which had low phytotoxic effect to cause small necrosis against several weedy and crop plant species [149]. The fungus Diaporthe sp. SCSIO 41011 derived from mangrove plant R. stylosa, afforded two metabolites, epi-isochromophilone II (300) and isochromophilone $\mathrm{D}$ (301). Compound 300 displayed cytotoxicities against ACHN, OS-RC-2, and 786-O cells with $\mathrm{IC}_{50}$ values between 3.0 and $4.4 \mu \mathrm{M}$, and 301 had an $\mathrm{IC}_{50}$ of $8.9 \mu \mathrm{M}$ against 786-O cancer cells [150]. The structures of unclassified polyketides (299-301) are shown in Figure 16.

\subsection{Terpenoids}

$(1 R, 2 R, 4 R)$-Trihydroxy-p-menthane (302) was isolated from Diaporthe sp. SXZ-19, and displayed weak cytotoxicity on HCT 116 cells [134]. Two new $\alpha$-pyrones, gulypyrones A (303) and B (304), were extracted from D. gulyae. Both of them showed phytotoxic activities and gulypyrone A caused necrosis against Helianthus annuus plantlets [149]. A pentaketide monoterpenoid, nectriapyrone (173), was isolated from culture of D. Kongii, showing phytotoxic activity [135]. A new brasilane-type sesquiterpenoid, diaporol R (305) was produced by an endophytic fungus Diaporthe sp. isolated from leaves of $R$. stylosa. Diaporol $\mathrm{R}$ had moderate cytotoxic effect on SW480 cancer cells and provided an $\mathrm{IC}_{50}$ value at $8.72 \pm 1.32 \mu \mathrm{M}$ [151]. Eremofortin F (306) was obtained from endophytic fungus Diaporthe sp. SNB-GSS10 of Sabicea cinerea. It showed cytotoxic activity against KB and MRC5 cells with $\mathrm{IC}_{50}$ values of 13.9 and $12.2 \mu \mathrm{M}$ [152]. Two new eremophilanes, lithocarins B (307) and C (308), were extracted from D. lithocarpus A740, an endophytic fungus isolated from Morinda officinalis. These compounds displayed low cytotoxicities against SF-268, MCF-7, HepG-2, and A549 tumor cells with $\mathrm{IC}_{50}$ values between 37.68-97.71 $\mu \mathrm{M}$ [153]. The new triterpenoid, 19-nor-lanosta-5(10),6,8,24-tetraene- 1 $\alpha, 3 \beta, 12 \beta, 22 S$-tetraol (309), was obtained from Diaporthe sp. LG23 of the Chinese medicinal plant Mahonia fortunei, and displayed antibacterial activity against both Gram-positive and Gram-negative bacteria [154]. The structures of terpenoids (302-309) are shown in Figure 17.

\subsection{Steriods}

Only two steroids, 3 $\beta, 5 \alpha, 9 \alpha$-trihydroxy-(22E,24R)-ergosta-7,22-dien-6-one (216) and chaxine C (310) (Figure 17), were isolated from Diaporthe sp. LG23, showing antibacterial activities against $B$. subtilis with streptomycin as a positive control [154]. 
<smiles>CC(C)C1(O)CC[C@@](C)(O)[C@H](O)C1</smiles>

302<smiles>COc1cc(=O)oc(C(C)C(C)C)c1C</smiles>

303<smiles>COc1cc(/C(C)=C/CO)oc(=O)c1C</smiles>

304<smiles>CC1=C(C=O)C(O)C[C@H]2C(C)CCCC12C</smiles>

305<smiles>C=C(C)[C@H]1C[C@]2(CO)C(=CC1=O)CC[C@@H](OC(=O)C(C)C(O)/C=C/C=C/CC(C)O)[C@@H]2C</smiles>

306<smiles>C=C(C)[C@H]1C[C@]2(C)C(=CC1=O)CC[C@@H](OC(=O)C(C)C(O)/C=C/C=C/CC(C)OC(C)=O)[C@@H]2C</smiles>

307<smiles>C/C=C\C(=O)O[C@H]1CCC2=CC(=O)C(=C(C)C)C[C@]2(C)[C@@H]1C</smiles><smiles>CC(C)[C@H](C)/C=C/[C@H](C)[C@H]1CC[C@H]2/C(=C/C(=O)O[C@]3(C)CCC=CC3=O)C(=O)CC[C@@]21C</smiles>
309<smiles>CCC[C@H](OC(=O)/C=C\C=C/[C@H](O)[C@H](O)[C@H](C)COC(=O)c1ccc(O)cc1)C(C)C</smiles>

Figure 17. Chemical structures of compounds 302-314 from Diaporthe.

\subsection{Ten-Membered Lactones}

Ten-membered lactones always have anti-tumor, anti-inflammatory, anti-viral, antibacterial and other pharmacological activities, exhibiting important medical value in clinical practice [166]. Phomolide C (311) from Diaporthe sp. of Aucuba japonica var. borealis, inhibited the proliferation of human colon adenocarcinoma cells with concentration of $50 \mu \mathrm{g} / \mathrm{mL}$ [155]. The endophytic fungus D. terebinthifolii GG3F6 derived from medicinal plant Glycyrrhiza glabra, afforded two known compounds, xylarolide (312) and phomolide $\mathrm{G}$ (313). Compound 312 had cytotoxicity in vitro against cancer cells MIAPaCa-2, HCT-116 and T47D cancer cells with $\mathrm{IC}_{50}$ values of 38,100 , and $7 \mu \mathrm{M}$ and showed notable antimicrobial activity against $C$. albicans and Yersinia enterocolitica with $\mathrm{IC}_{50}$ values at 78.8 and $72.1 \mu \mathrm{M}$. Moreover, Compound 313 showed an $\mathrm{IC}_{50}$ value of $69.2 \mu \mathrm{M}$ against $Y$. enterocolitica [156]. A novel metabolite, named xylarolide A (314), was isolated from the fungus Diaporthe sp. of D. inoxia. Compound 314 had remarkable cytotoxicities against MIAPaCa-2 and PC-3 cancer cells with $\mathrm{IC}_{50}$ values between $14-32 \mu \mathrm{M}$, and also showed antioxidant activity on DPPH radical scavenging effect $\left(\mathrm{EC}_{50}=10.3 \mu \mathrm{M}\right)$ [133]. The structures of four ten-membered lactones (311-314) are shown in Figure 17.

\subsection{Alkaloids}

18-Des-hydroxy cytochalasin $\mathrm{H}$ (315) was obtained from endophytic fungus $D$. phaseolorum-92C of Combretum lanceolatum. This compound inhibited leishmanicidal activity, displayed moderate antioxidant activity, and had cytotoxic activity against the breast cancer cells MDA-MB-231 and MCF-7 [157]. A series of the cytochalasins were extracted from Diaporthe sp. GDG-118 of Sophora tonkinensis, including 21-acetoxycytochalasins $\mathrm{J}_{2}(316)$ and $\mathrm{J}_{3}$ (317), 7-acetoxycytochalasin $\mathrm{H}$ (319), and cytochalasins $\mathrm{J}_{3}$ (318), H (230), J (231), and E (320). All isolated metabolites showed different degrees of antifungal activities against Alternaria oleracea, Pestalotiopsis theae, Colletotrichum capsici, and Ceratocystis paradoxa with MIC values 
of $1.56-100 \mu \mathrm{g} / \mathrm{mL}$, and antibacterial activities against Gram-positive bacteria (B. subtilis, B. megaterium and Bacillus anthraci) and Gram-negative bacteria (Proteus vuigaris, E. coli and Salmonella paratyphi B) with MIC values in the range of $12.5-100 \mu \mathrm{g} / \mathrm{mL}$ [158]. The fungus Diaporthe sp. GZU-1021 yielded cytochalasin H (230) and 21-O-deacetyl-L-696,474 (321), which showed anti-inflammatory activities by inhibiting NO production in RAW 264.7 cells with $\mathrm{IC}_{50}$ values of 1.94 and $7.35 \mu \mathrm{M}$ [126]. Cordysinin A (322) was derived from endophytic fungus $D$. arecae of Kandelia obovate. It showed anti-angiogenic activity against the human endothelial progenitor cells (EPCs) with $\mathrm{IC}_{50}$ value of $15.1 \pm 0.2 \mu \mathrm{g} / \mathrm{mL}$ [159]. Further research led to the identification of 5-deoxybostrycoidin (323) and fusaristatin A (241) from D. phaseolorum SKS019 of mangrove plant A. ilicifolius. Compound 323 showed cytotoxic activity against MDA-MB-435 and NCI-H460 with IC 50 values at 5.32 and $6.57 \mu \mathrm{M}$, and the $\mathrm{IC}_{50}$ value of 241 was $8.15 \mu \mathrm{M}$ on MDA-MB-435 [160]. A new carboxamide, vochysiamide B (324), was extracted from new species D. vochysiae LGMF1583, which displayed antibacterial activity on the Gram-negative bacterium Klebsiella pneumoniae (KPC) with MIC value at $80 \mu \mathrm{g} / \mathrm{mL}$ and showed cytotoxic activity against $\mathrm{A} 549\left(\mathrm{EC}_{50}=86.4 \mu \mathrm{M}\right)$ and PC3 $\left(\mathrm{EC}_{50}=40.25 \mu \mathrm{M}\right)$ [143]. Four compounds, diaporisoindoles A (325), B (326), D (327), and E (328), were obtained from an endophytic fungus Diaporthe sp. SYSU-HQ3. They all showed anti-inflammatory activities by reducing NO production with $\mathrm{IC}_{50}$ values of 22.7, 18.2, 8.9, and $8.3 \mu \mathrm{M}$, respectively [148]. Diaporisoindole D (327) also exhibited inhibitory activity towards M. tuberculosis protein tyrosine phosphatase B $(\mathrm{MptpB})\left(\mathrm{IC}_{50}=4.2 \mu \mathrm{M}\right)$ [147]. Phomopsin F (329) was isolated from $D$. toxica, and showed cytotoxic activity against HepG2 cells [161]. The structures of alkaloids (315-329) are shown in Figure 18.

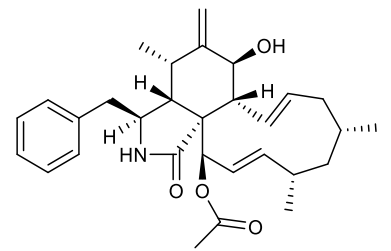

315

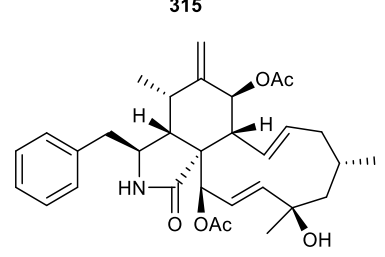

319

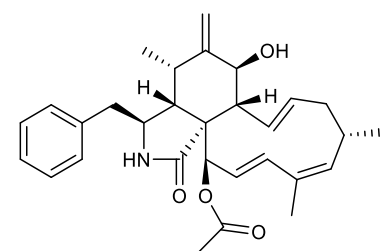

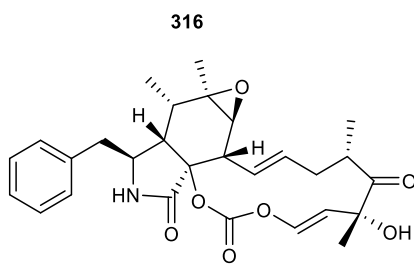

320

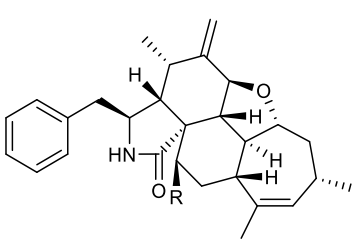

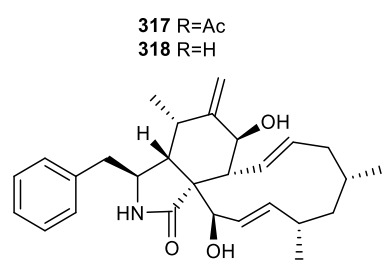

321

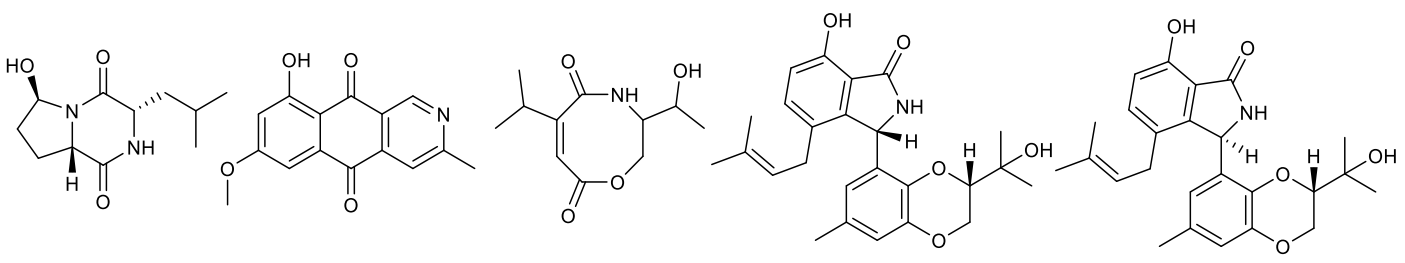<smiles>COCC1(C(C)(C)O)COc2cc(C)cc(C3(OC)NC(=O)c4c(O)ccc(CC=C(C)C)c43)c2O1</smiles>

327

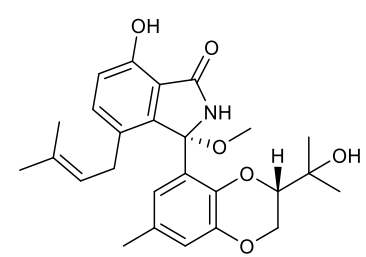

328
325

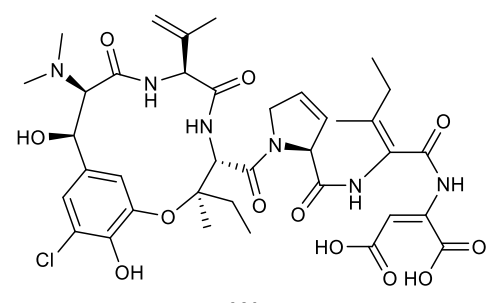

329

Figure 18. Chemical structures of compounds 315-329 from Diaporthe. 


\subsection{Fatty Acids}

Fatty acids are simple linear compounds that play an important role in the synthesis and catabolism of organisms [167]. Over here, six fatty acids are reported from Diaporthe. The fungus $D$. phaseolorum derived from Laguncularia racemose, afforded 3-hydroxypropionic acid (330), which showed antimicrobial activity against S. aureus and S. typhi [162]. A phytotoxic metabolite, 3-nitropropionic acid (331), was isolated from D. gulyae. Compound 331 was notably active in causing necroses on several weedy and crop plant species [149]. Two new fatty acids, diapolic acids A and B (332 and 333), were isolated from endophytic fungus $D$. terebinthifolii. They had moderate antibacterial activities against $Y$. enterocolitica with $\mathrm{IC}_{50}$ values of 78.4 and $73.4 \mu \mathrm{M}$ [156]. Studies of the strain Diaporthe sp. JC-J7 from stems of Dendrobium nobile led to the isolation of a new compound, diaporthsin E (334). It showed low antihyperlipidemic activity on triglycerides (TG) in steatotic L-02 cells with the inhibition rate of $26 \%$ at the concentration of $5 \mu \mathrm{g} / \mathrm{mL}$ [163]. The novel anti-candidal metabolite, 3-hydroxy-5-methoxyhex-5-ene-2,4-dione (335), was derived from Diaporthe sp. ED2 of medicinal herb Orthosiphon stamieus Benth. It showed antifungal activity against C. albicans with MIC value of $3.1 \mu \mathrm{g} / \mathrm{mL}$ [164]. The structures of fatty acids (330-335) are shown in Figure 19.<smiles>C=C(OC)C(=O)C(O)C(C)=O</smiles>

Figure 19. Chemical structures of compounds 330-335 from Diaporthe.

\section{Characteristics of Bioactive Secondary Metabolites from the Genus Diaporthe and Anamorph Phomopsis}

In this paper, a total of 335 bioactive compounds from the genus Diaporthe and Phomopsis are summarized. There are 106 secondary metabolites from Diaporthe and 246 ones from Phomopsis, in which 17 compounds were obtained from both of Diaporthe and Phomopsis. These compounds are classified into polyketides, terpenoids, steroids, macrolides, tenmembered lactones, alkaloids, flavonoids, and fatty acids. As seen in Figure 20, about two thirds of all compounds reported from Diaporthe and Phomopsis are refered to polyketides, accounting for $63 \%$ and $70 \%$, respectively. Moreover, terpenoids $(8 \%, 15 \%)$, alkaloids $(17 \%$, $6 \%)$, and steroids $(2 \%, 4 \%)$ were also produced by both of Diaporthe and Phomopsis. It is worth noting that fatty acids $(6 \%)$ and ten-membered lactones $(4 \%)$ are only reported from Diaporthe, while flavonoids (2\%) and macrolides (3\%) are only found in Phomopsis. Polyketides, as the largest member of the metabolites, are widely used in the field of medicine and play an important role in the treatment of cancer diseases.

The various bioactivities of the compounds isolated from Diaporthe and Phomopsis are presented in Figure 21, mainly containing cytotoxic, antibacterial, antifungal, antiviral, anti-inflammatory, antioxidant, antialgae, enzyme inhibition, and phytotoxic activities. Most of compounds have at least one kind of bioactivities. As seen in Figure 21 and Tables 1 and 2, secondary metabolites of Diaporthe and Phomopsis mainly exhibit cytotoxic, antibacterial and antifungal activities, accounting for $73 \%$ of all compounds, with 56 in Diaporthe and 200 from Phomopsis. Interestingly, in recent years, more and more compounds with anti-inflammatory, antioxidant and enzyme inhibitory activities have been studied in important human diseases. 


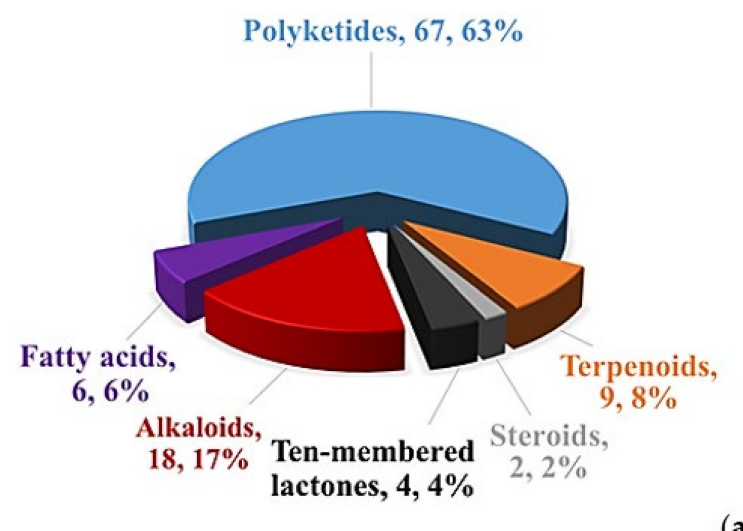

(a)

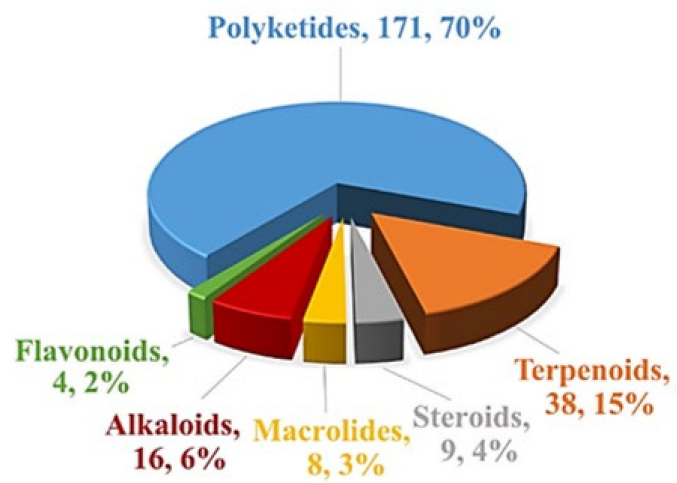

(b)

Figure 20. (a) The proportion of structural types of bioactive compounds from Diaporthe; (b) The proportion of structural types of bioactive compounds from Phomopsis.

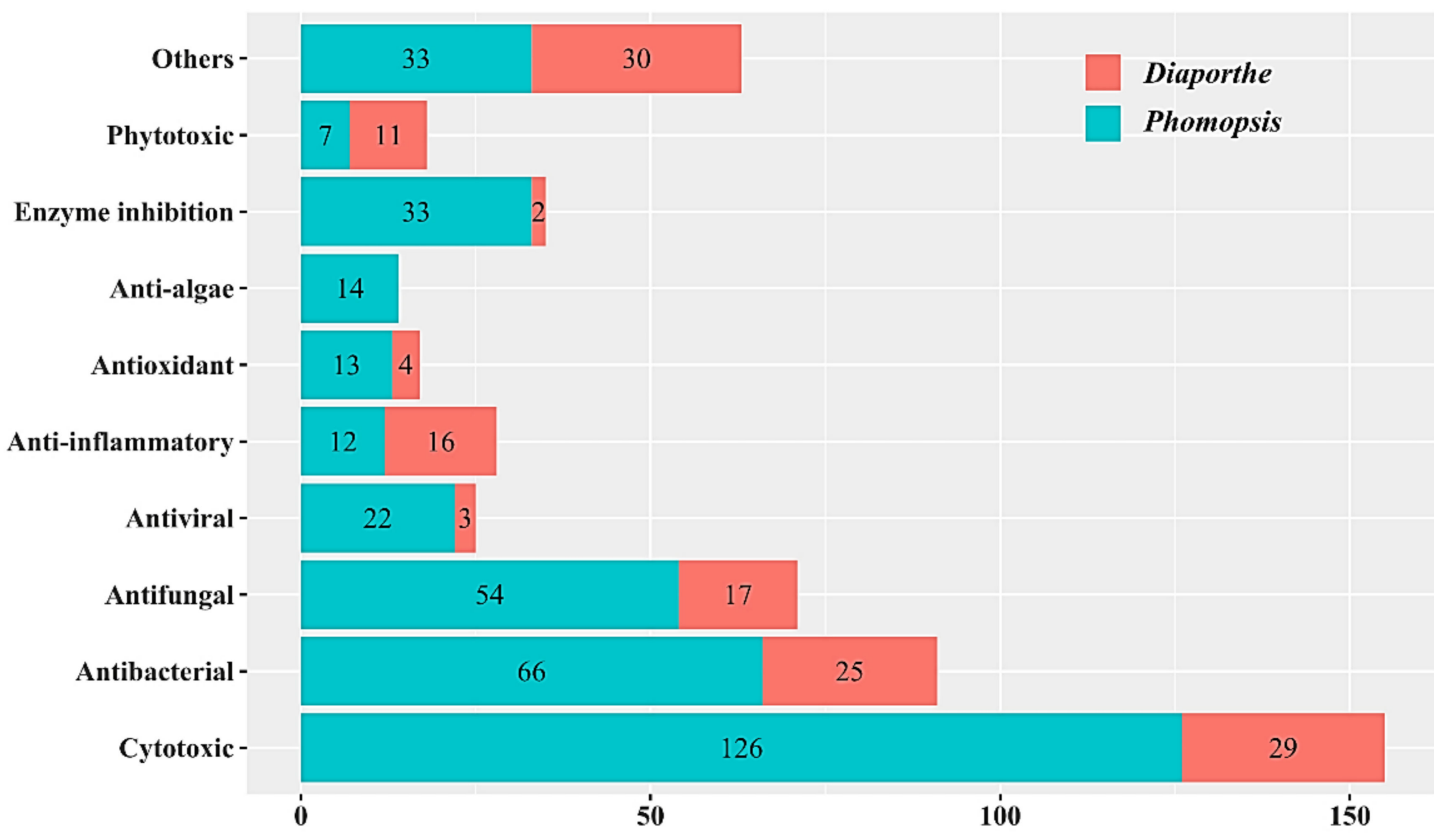

Figure 21. The distribution of main bioactivities of compounds isolated from Diaporthe and Phomopsis.

\section{Conclusions}

This review presents the diverse chemical structures and bioactivities of $335 \mathrm{com}$ pounds isolated from 26 known species and various unidentified species of the genus Dia- 
porthe and its anamorph Phomopsis between 2010-2019. Here, we can see from Tables 1 and 2, among all of the reported compounds, there are 236 (accounting for about 70\%) and 92 (about 27\%) compounds derived only from terrestrial and marine environments (including mangroves, sediments, deep-sea fungi and marine animals), respectively. In addition, only one compound is obtained from both of terrestrial and marine environments. In contrast, six compounds are not mentioned with their habitats in the literature. Polyketides represent the main chemical population, accounting for $64 \%$. About $73 \%$ of all metabolites possess cytotoxic, antibacterial, and antifungal activities. The species named as Phomopsis significantly produce much more compounds than Diaporthe, and most strains have not yet been identified at the species level. In conclusion, these results illustrate that the metabolic resources of Diaporthe and Phomopsis are of great value and deserved to conduct further research. Interestingly, in the past three years, there have been more reports on the secondary metabolites of the fungi in Diaporthe and Phomopsis than before, displaying an increasing trend, which indicates that Diaporthe and Phomopsis are regarded as important sources for discovering new natural bioactive substances.

In the past many years, lots of interesting fungal bioactive metabolites had been widely developed into new drugs, like antibiotics. Although most compounds obtained from Diaporthe and Phomopsis fungi had been studied on their isolation, structures, and activities, the in-depth research on pharmacological mechanisms and development of potent active compounds in drugs are still less. According to current studies, some compounds with remarkable bioactivities may serve as potential drug candidates in the future, such as cytotoxic altersolanol A and PM181110, and antimicrobial dicerandrol A. In order to ascertain the therapeutic potential of these compounds, further studies of pharmacological and producing mechanisms are required.

The fungal species in Diaporthe and Phomopsis have been considered to be important sources that can produce diverse and novel bioactive metabolites, which has attracted many natural product chemists and pharmacologists to study in recent years. The metabolites produced by Diaporthe and Phomopsis have rich biological activities, which is enough to show the importance of its metabolic resources. Nowadays, many fungi produce interesting bioactive metabolites that have been studied for their biosynthesis pathway, while similar studies in Diaporthe and Phomopsis are performed relatively less often. In the following work, the microbial biosynthesis pathway might be considered for further developing valuable products from Diaporthe or Phomopsis, which are hoped to be used as drug molecules for disease treatment. However, it cannot be ignored that Diaporthe or Phomopsis are important plant pathogens which might cause a wide range of plant host diseases and even serious human pathogens. In the future work, we should also focus on the role of metabolites produced by these pathogens, as well as the relationships with their hosts.

Author Contributions: Manuscript preparation, T.-C.X.; prepared figures, tables, and analyzed data, Y.-H.L.; data proofreading and manuscript revision, J.-F.W., Z.-Q.S., Y.-G.H., S.-S.L., and C.-S.L.; manuscript conception and revision, S.-H.W. All authors have read and agreed to the published version of the manuscript.

Funding: This research was funded by the National Natural Science Foundation of China (No. 81860634), Applied Basic Research Key Project of Yunnan Province (No. 202001BB050029), Major Science and Technology Projects of Yunnan Province (Digitalization, development and application of biotic resource, 202002AA100007), and Project of Innovative Research Team of Yunnan Province (202005AE160005).

Institutional Review Board Statement: Not applicable.

Informed Consent Statement: Not applicable.

Data Availability Statement: All data in this article is openly available without any restrictions.

Conflicts of Interest: The authors declare no conflict of interest. 


\section{References}

1. Guarnaccia, V.; Groenewald, J.Z.; Woodhall, J.; Armengol, J.; Cinelli, T.; Eichmeier, A.; Ezra, D.; Fontaine, F.; Gramaje, D.; Gutierrez-Aguirregabiria, A.; et al. Diaporthe diversity and pathogenicity revealed from a broad survey of grapevine diseases in Europe. Persoonia 2018, 40, 135-153. [CrossRef]

2. Dissanayake, A.J.; Chen, Y.Y.; Liu, J.K.J. Unravelling Diaporthe species associated with woody hosts from karst formations (Guizhou) in China. J. Fungi 2020, 6, 251. [CrossRef]

3. Gomes, R.R.; Glienke, C.; Videira, S.I.R.; Lombard, L.; Groenewald, J.Z.; Crous, P.W. Diaporthe: A genus of endophytic, saprobic and plant pathogenic fungi. Persoonia 2013,31,1-41. [CrossRef]

4. Udayanga, D.; Castlebury, L.A.; Rossman, A.Y.; Chukeatirote, E.; Hyde, K.D. Insights into the genus Diaporthe: Phylogenetic species delimitation in the D. eres species complex. Fungal Divers. 2014, 67, 203-229. [CrossRef]

5. Gao, Y.; Liu, F.; Duan, W.; Crous, P.W.; Cai, L. Diaporthe is paraphyletic. IMA Fungus 2017, 8, 153-187. [CrossRef]

6. Santos, J.M.; Correia, V.G.; Phillips, A.J.L. Primers for mating-type diagnosis in Diaporthe and Phomopsis: Their use in teleomorph induction in vitro and biological species definition. Fungal Biol. 2010, 114, 255-270. [CrossRef]

7. Gong, J.L.; Lu, Y.; Wu, W.H.; He, C.P.; Liang, Y.Q.; Huang, X.; Zheng, J.L.; Xi, J.G.; Tang, S.B.; Yi, K.X. First report of Phomopsis heveicola (anamorph of Diaporthe tulliensis) causing leaf blight of Coffee (Coffea arabica) in China. Plant Dis. 2020, 104, 570-571. [CrossRef]

8. Rehner, S.A.; Uecker, F.A. Nuclear ribosomal internal transcribed spacer phylogeny and host diversity in the coelomycete Phomopsis. Can. J. Bot. 1994, 72, 1666-1674. [CrossRef]

9. Santos, J.M.; Vrandecic, K.; Cosic, J.; Duvnjak, T.; Phillips, A.J.L. Resolving the Diaporthe species occurring on soybean in Croatia. Persoonia 2011, 27, 9-19. [CrossRef] [PubMed]

10. Zhou, H.; Hou, C.L. Three new species of Diaporthe from China based on morphological characters and DNA sequence data analyses. Phytotaxa 2019, 422, 157-174. [CrossRef]

11. Leon, M.; Berbegal, M.; Rodriguez-Reina, J.M.; Elena, G.; Abad-Campos, P.; Ramon-Albalat, A.; Olmo, D.; Vicent, A.; Luque, J.; Miarnau, X.; et al. Identification and characterization of Diaporthe spp. associated with twig cankers and shoot blight of almonds in Spain. Agronomy 2020, 10, 1062. [CrossRef]

12. Rossman, A.Y.; Adams, G.C.; Cannon, P.F.; Castlebury, L.A.; Crous, P.W.; Gryzenhout, M.; Jaklitsch, W.M.; Mejia, L.C.; Stoykov, D.; Udayanga, D.; et al. Recommendations of generic names in Diaporthales competing for protection or use. IMA Fungus 2015, 6, 145-154. [CrossRef] [PubMed]

13. Udayanga, D.; Liu, X.; McKenzie, E.H.C.; Chukeatirote, E.; Bahkali, A.H.A.; Hyde, K.D. The genus Phomopsis: Biology, applications, species concepts and names of common phytopathogens. Fungal Divers. 2011, 50, 189-225. [CrossRef]

14. Santos, L.; Alves, A.; Alves, R. Evaluating multi-locus phylogenies for species boundaries determination in the genus Diaporthe. PeerJ 2017, 5. [CrossRef]

15. Lin, X.; Huang, Y.J.; Fang, M.J.; Wang, J.F.; Zheng, Z.H.; Su, W.J. Cytotoxic and antimicrobial metabolites from marine lignicolous fungi, Diaporthe sp. FEMS Microbiol. Lett. 2005, 251, 53-58. [CrossRef]

16. Silva, G.H.; Teles, H.L.; Zanardi, L.M.; Marx Young, M.C.; Eberlin, M.N.; Hadad, R.; Pfenning, L.H.; Costa-Neto, C.M.; CastroGamboa, I.; Bolzani, V.d.S.; et al. Cadinane sesquiterpenoids of Phomopsis cassiae, an endophytic fungus associated with Cassia spectabilis (Leguminosae). Phytochemistry 2006, 67, 1964-1969. [CrossRef]

17. Niaz, S.I.; Khan, D.; Naz, R.; Safdar, K.; Ul Abidin, S.Z.; Khan, I.U.; Gul, R.; Khan, W.U.; Khan, M.A.U.; Lan, L. Antimicrobial and antioxidant chlorinated azaphilones from mangrove Diaporthe perseae sp. isolated from the stem of Chinese mangrove Pongamia pinnata. J. Asian Nat. Prod. Res. 2020. [CrossRef]

18. Yang, Z.J.; Zhang, Y.F.; Wu, K.; Xu, Y.X.; Meng, X.G.; Jiang, Z.T.; Ge, M.; Shao, L. New azaphilones, phomopsones A-C with biological activities from an endophytic fungus Phomopsis sp. CGMCC No.5416. Fitoterapia 2020, 145. [CrossRef]

19. Da Rosa, B.V.; Kuhn, K.R.; Ugalde, G.A.; Zabot, G.L.; Kuhn, R.C. Antioxidant compounds extracted from Diaporthe schini using supercritical $\mathrm{CO}_{2}$ plus cosolvent. Bioprocess Biosyst. Eng. 2020, 43, 133-141. [CrossRef]

20. Fan, M.M.; Xiang, G.; Chen, J.W.; Gao, J.; Xue, W.W.; Wang, Y.X.; Li, W.H.; Zhou, L.; Jiao, R.H.; Shen, Y.; et al. Libertellenone M, a diterpene derived from an endophytic fungus Phomopsis sp. S12, protects against DSS-induced colitis via inhibiting both nuclear translocation of NF-kB and NLRP3 inflammasome activation. Int. Immunopharmacol. 2020, 80. [CrossRef]

21. Tsantrizos, Y.S.; Ogilvie, K.K.; Watson, A.K. Phytotoxic metabolites of Phomopsis convolvulus, a host-specific pathogen of field bindweed. Can. J. Chem. 1992, 70, 2276-2284. [CrossRef]

22. Zhang, C.W.; Ondeyka, J.G.; Herath, K.B.; Guan, Z.Q.; Collado, J.; Platas, G.; Pelaez, F.; Leavitt, P.S.; Gurnett, A.; Nare, B.; et al. Tenellones A and B from a Diaporthe sp.: Two highly substituted benzophenone inhibitors of parasite cGMP-dependent protein kinase activity. J. Nat. Prod. 2005, 68, 611-613. [CrossRef] [PubMed]

23. Yang, H.Y.; Gao, Y.H.; Niu, D.Y.; Yang, L.Y.; Gao, X.M.; Du, G.; Hu, Q.F. Xanthone derivatives from the fermentation products of an endophytic fungus Phomopsis sp. Fitoterapia 2013, 91, 189-193. [CrossRef] [PubMed]

24. Yang, J.X.; Qiu, S.X.; She, Z.G.; Lin, Y.C. A new xanthone derivative from the marine fungus Phomopsis sp. (No. SK7RN3G1). Chem. Nat. Compd. 2013, 49, 31-33. [CrossRef]

25. Yuan, L.; Huang, W.; Du, G.; Gao, X.; Yang, H.; Hu, Q.; Ma, Y. Isolation of xanthones from the fermentation products of the endophytic fungus of Phomopsis amygdali. Chem. Nat. Compd. 2015, 51, 460-463. [CrossRef] 
26. Huang, R.; Ma, K.X.; Xie, X.S.; Wang, T.; Wu, S.H. Secondary metabolites of an endophytic fungus Phomopsis sp. Chem. Nat. Compd. 2015, 51, 392-394. [CrossRef]

27. Yuan, L.; Huang, W.Z.; Zhou, K.; Wang, Y.D.; Dong, W.; Lou, J.; Li, L.M.; Du, G.; Yang, H.Y.; Ma, Y.H.; et al. Xanthones from the fermentation products of an endophytic fungus Phomopsis sp. Heterocycles 2015, 91, 381-387. [CrossRef]

28. Yang, Y.; Yang, H.; Li, Y.; Ye, Y.; Hu, Q.; Gao, X.; Du, G. A new xanthone from the fermentation products of endophytic fungus of Phomopsis species. Asian J. Chem. 2014, 26, 4591-4593. [CrossRef]

29. Hu, Q.; Yang, Y.; Yang, S.; Cao, H.; Chunyang, M.; Yang, H.; Gao, X.; Du, G. Xanthones from the fermentation products of the endophytic fungus of Phomopsis amygdali. Chem. Nat. Compd. 2015, 51, 456-459. [CrossRef]

30. Huang, Z.; Yang, J.; Lei, F.; She, Z.; Lin, Y. A new xanthone O-glycoside from the mangrove endophytic fungus Phomopsis sp. Chem. Nat. Compd. 2013, 49, 27-30. [CrossRef]

31. Roensberg, D.; Debbab, A.; Mandi, A.; Vasylyeva, V.; Boehler, P.; Stork, B.; Engelke, L.; Hamacher, A.; Sawadogo, R.; Diederich, M.; et al. Pro-apoptotic and immunostimulatory tetrahydroxanthone dimers from the endophytic fungus Phomopsis longicolla. J. Org. Chem. 2013, 78, 12409-12425. [CrossRef] [PubMed]

32. Shiono, Y.; Sasaki, T.; Shibuya, F.; Yasuda, Y.; Koseki, T.; Supratman, U. Isolation of a phomoxanthone A derivative, a new metabolite of tetrahydroxanthone, from a Phomopsis sp. isolated from the mangrove, Rhizhopora mucronata. Nat. Prod. Commun. 2013, 8, 1735-1737. [CrossRef] [PubMed]

33. Meixiang, H.; Jing, L.; Lan, L.; Sheng, Y.; Jun, W.; Yongcheng, L.J.M.D. Phomopsichin A-D; four new chromone derivatives from mangrove endophytic fungus Phomopsis sp. 33\#. Mar. Drugs 2016, 14, 215. [CrossRef]

34. Lim, C.; Kim, J.; Choi, J.N.; Ponnusamy, K.; Jeon, Y.; Kim, S.U.; Kim, J.G.; Lee, C.H. Identification, fermentation, and bioactivity against xanthomonas oryzae of antimicrobial metabolites isolated from Phomopsis longicolla S1B4. J. Microbiol. Biotechnol. 2010, 20, 494-500. [CrossRef]

35. Ding, B.; Yuan, J.; Huang, X.; Wen, W.; Zhu, X.; Liu, Y.; Li, H.; Lu, Y.; He, L.; Tan, H.J.M.D. New dimeric members of the phomoxanthone family: Phomolactonexanthones A, B and deacetylphomoxanthone C isolated from the fungus Phomopsis sp. Mar. Drugs 2013, 11, 4961-4972. [CrossRef]

36. Ding, B.; Wang, Z.; Xia, G.; Huang, X.; Xu, F.; Chen, W.; She, Z. Three new chromone derivatives produced by Phomopsis sp. HNY29-2B from Acanthus ilicifolius linn. Chin. J. Chem. 2017, 35, 1889-1893. [CrossRef]

37. Wu, Q.; Guo, Y.; Guo, Z.K.; Chu, Y.L.; Wang, T.; Tan, R.X. Two new cytosporones from the culture of endophytic Phomopsis sp. Chem. Nat. Compd. 2013, 48, 938-941. [CrossRef]

38. Hu, H.B.; Luo, Y.F.; Wang, P.; Wang, W.J.; Wu, J. Xanthone-derived polyketides from the Thai mangrove endophytic fungus Phomopsis sp. xy21. Fitoterapia 2018, 131, 265-271. [CrossRef]

39. Yang, J.X.; Qiu, S.; She, Z.; Lin, Y. A new isochroman derivative from the marine fungus Phomopsis sp. (No. Gx-4). Chem. Nat. Compd. 2014, 50, 424-426. [CrossRef]

40. Ahmed, I.; Hussain, H.; Schulz, B.; Draeger, S.; Padula, D.; Pescitelli, G.; van Ree, T.; Krohn, K. Three new antimicrobial metabolites from the endophytic fungus Phomopsis sp. Eur. J. Org. Chem. 2011, 2011, 2867-2873. [CrossRef]

41. Yang, Z.; Wu, K.; Xu, Y.; Xia, X.; Wang, X.; Ge, M.; Shao, L. Three novel chromanones with biological activities from the endophytic fungus Phomopsis CGMCC No. 5416. J. Antibiot. 2020, 73, 194-199. [CrossRef] [PubMed]

42. Huang, Z.; Yang, R.; Guo, Z.; She, Z.; Lin, Y. A new naphtho- $\gamma$-pyrone from mangrove endophytic fungus ZSU-H26. Chem. Nat. Compd. 2010, 46, 15-18. [CrossRef]

43. Yang, J.; Xu, F.; Huang, C.; Li, J.; She, Z.; Pei, Z.; Lin, Y. Metabolites from the mangrove endophytic fungus Phomopsis sp. (\#zsu-H76). Eur. J. Org. Chem. 2010, 2010, 3692-3695. [CrossRef]

44. Yang, J.X.; Chen, Y.; Huang, C.; She, Z.; Lin, Y. A new isochroman derivative from the marine fungus Phomopsis sp. (No. ZH-111). Chem. Nat. Compd. 2011, 47, 13-16. [CrossRef]

45. Tang, J.W.; Wang, W.G.; Li, A.; Yan, B.C.; Chen, R.; Li, X.N.; Du, X.; Sun, H.D.; Pu, J.X. Polyketides from the endophytic fungus Phomopsis sp. sh917 by using the one strain/many compounds strategy. Tetrahedron 2017, 73, 3577-3584. [CrossRef]

46. Adelin, E.; Martin, M.T.; Cortial, S.; Retailleau, P.; Lumyong, S.; Ouazzani, J. Bioactive polyketides isolated from agar-supported fermentation of Phomopsis sp. CMU-LMA, taking advantage of the scale-up device, Platotex. Phytochemistry 2013, 93, 170-175. [CrossRef]

47. Tao, M.H.; Chen, Y.C.; Wei, X.Y.; Tan, J.W.; Zhang, W.M. Chemical constituents of the endophytic fungus Phomopsis sp. A240 isolated from Taxus chinensis var. mairei. Helv. Chim. Acta 2014, 97, 426-430. [CrossRef]

48. Talontsi, F.M.; Islam, M.T.; Facey, P.; Douanla-Meli, C.; von Tiedemann, A.; Laatsch, H. Depsidones and other constituents from Phomopsis sp. CAFT69 and its host plant Endodesmia calophylloides with potent inhibitory effect on motility of zoospores of grapevine pathogen Plasmopara viticola. Phytochem. Lett. 2012, 5, 657-664. [CrossRef]

49. Chapla, V.M.; Zeraik, M.L.; Ximenes, V.F.; Zanardi, L.M.; Lopes, M.N.; Cavalheiro, A.J.; Silva, D.H.S.; Young, M.C.M.; da Fonseca, L.M.; Bolzani, V.S.; et al. Bioactive secondary metabolites from Phomopsis sp., an endophytic fungus from Senna spectabilis. Molecules 2014, 19, 6597-6608. [CrossRef]

50. Zhang, W.; Xu, L.; Yang, L.; Huang, Y.; Li, S.; Shen, Y. Phomopsidone A, a novel depsidone metabolite from the mangrove endophytic fungus Phomopsis sp. A123. Fitoterapia 2014, 96, 146-151. [CrossRef]

51. Kornsakulkarn, J.; Somyong, W.; Supothina, S.; Boonyuen, N.; Thongpanchang, C. Bioactive oxygen-bridged cyclooctadienes from endophytic fungus Phomopsis sp. BCC 45011. Tetrahedron 2015, 71, 9112-9116. [CrossRef] 
52. Xu, J.L.; Liu, Z.M.; Chen, Y.C.; Tan, H.B.; Li, H.H.; Li, S.N.; Guo, H.; Huang, Z.L.; Gao, X.X.; Liu, H.X.; et al. Lithocarols A-F, six tenellone derivatives from the deep-sea derived fungus Phomopsis lithocarpus FS508. Bioorg. Chem. 2019, 87, 728-735. [CrossRef] [PubMed]

53. Du, G.; Wang, Z.C.; Hu, W.Y.; Yan, K.L.; Wang, X.L.; Yang, H.M.; Yang, H.Y.; Gao, Y.H.; Liu, Q.; Hu, Q.F. Three new 3-methyl-2arylbenzofurans from the fermentation products of an endophytic fungus Phomopsis sp. and their anti-TMV activity. Phytochem. Lett. 2017, 21, 287-290. [CrossRef]

54. Song, H.C.; Qin, D.; Han, M.J.; Wang, L.; Zhang, K.; Dong, J.Y. Bioactive 2-pyrone metabolites from an endophytic Phomopsis asparagi SWUKJ5.2020 of Kadsura angustifolia. Phytochem. Lett. 2017, 22, 235-240. [CrossRef]

55. Hussain, H.; Ahmed, I.; Schulz, B.; Draeger, S.; Krohn, K. Pyrenocines J-M: Four new pyrenocines from the endophytic fungus, Phomopsis sp. Fitoterapia 2012, 83, 523-526. [CrossRef]

56. Yang, Z.; Ding, J.; Ding, K.; Chen, D.; Cen, S.; Ge, M. Phomonaphthalenone A: A novel dihydronaphthalenone with anti-HIV activity from Phomopsis sp. HCCB04730. Phytochem. Lett. 2013, 6, 257-260. [CrossRef]

57. Li, X.B.; Chen, G.Y.; Liu, R.J.; Zheng, C.J.; Song, X.M.; Han, C.R. A new biphenyl derivative from the mangrove endophytic fungus Phomopsis longicolla HL-2232. Nat. Prod. Res. 2017, 31, 2264-2267. [CrossRef]

58. Mishra, P.D.; Verekar, S.A.; Deshmukh, S.K.; Joshi, K.S.; Fiebig, H.H.; Kelter, G. Altersolanol A: A selective cytotoxic anthraquinone from a Phomopsis sp. Lett. Appl. Microbiol. 2015, 60, 387-391. [CrossRef]

59. Evidente, A.; Rodeva, R.; Andolfi, A.; Stoyanova, Z.; Perrone, C.; Motta, A. Phytotoxic polyketides produced by Phomopsis foeniculi, a strain isolated from diseased Bulgarian fennel. Eur. J. Plant Pathol. 2011, 130, 173-182. [CrossRef]

60. Klaiklay, S.; Rukachaisirikul, V.; Phongpaichit, S.; Pakawatchai, C.; Saithong, S.; Buatong, J.; Preedanon, S.; Sakayaroj, J. Anthraquinone derivatives from the mangrove-derived fungus Phomopsis sp. PSU-MA214. Phytochem. Lett. 2012, 5, 738-742. [CrossRef]

61. Hussain, H.; Tchimene, M.K.; Ahmed, I.; Meier, K.; Steinert, M.; Draeger, S.; Schulz, B.; Krohn, K. Antimicrobial chemical constituents from the endophytic fungus Phomopsis sp. from Notobasis syriaca. Nat. Prod. Commun. 2011, 6, 1905-1906. [CrossRef] [PubMed]

62. Cai, R.; Chen, S.; Liu, Z.; Tan, C.; Huang, X.; She, Z. A new $\alpha$-pyrone from the mangrove endophytic fungus Phomopsis sp. HNY29-2B. Nat. Prod. Res. 2017, 31, 124-130. [CrossRef] [PubMed]

63. Krohn, K.; Farooq, U.; Hussain, H.; Ahmed, I.; Rheinheimer, J.; Draeger, S.; Schulz, B.; van Ree, T. Phomosines H-J, novel highly substituted biaryl ethers, isolated from the endophytic fungus Phomopsis sp. from Ligustrum vulgare. Nat. Prod. Commun. 2011, 6, 1907-1912. [CrossRef] [PubMed]

64. Hu, S.S.; Liang, M.J.; Mi, Q.L.; Chen, W.; Ling, J.; Chen, X.; Li, J.; Yang, G.Y.; Hu, Q.F.; Wang, W.G.; et al. Two new diphenyl ether derivatives from the fermentation products of the endophytic fungus Phomopsis asparagi. Chem. Nat. Compd. 2019, 55, 843-846. [CrossRef]

65. Gao, Y.H.; Zheng, R.; Li, J.; Kong, W.S.; Liu, X.; Ye, L.; Mi, Q.L.; Kong, W.S.; Zhou, M.; Yang, G.Y.; et al. Three new diphenyl ether derivatives from the fermentation products of an endophytic fungus Phomopsis fukushii. J. Asian Nat. Prod. Res. 2019, 21, 316-322. [CrossRef]

66. Li, Z.J.; Yang, H.Y.; Li, J.; Liu, X.; Ye, L.; Kong, W.S.; Tang, S.Y.; Du, G.; Liu, Z.H.; Zhou, M.; et al. Isopentylated diphenyl ether derivatives from the fermentation products of an endophytic fungus Phomopsis fukushii. J. Antibiot. 2018, 71, 359-362. [CrossRef]

67. Yang, H.Y.; Duan, Y.Q.; Yang, Y.K.; Liu, X.; Ye, L.; Mi, Q.L.; Kong, W.S.; Zhou, M.; Yang, G.Y.; Hu, Q.F.; et al. Two new diphenyl ether derivatives from the fermentation products of an endophytic fungus Phomopsis fukushii. Chem. Nat. Compd. 2019, 55, 428-431. [CrossRef]

68. Yang, H.Y.; Duan, Y.Q.; Yang, Y.K.; Li, J.; Liu, X.; Ye, L.; Mi, Q.L.; Kong, W.S.; Zhou, M.; Yang, G.Y.; et al. Three new napthalene derivatives from the endophytic fungus Phomopsis fukushii. Phytochem. Lett. 2017, 22, 266-269. [CrossRef]

69. Li, X.M.; Zeng, Y.C.; Chen, J.H.; Yang, Y.K.; Li, J.; Ye, L.; Du, G.; Zhou, M.; Hu, Q.F.; Guangyu, Y.; et al. Two new naphthalene derivatives from the fermentation products of an endophytic fungus Phomopsis sp. Chem. Nat. Compd. 2019, 55, 618-621. [CrossRef]

70. Xu, J.L.; Liu, H.X.; Chen, Y.C.; Tan, H.B.; Guo, H.; Xu, L.Q.; Li, S.N.; Huang, Z.L.; Li, H.H.; Gao, X.X.; et al. Highly substituted benzophenone aldehydes and eremophilane derivatives from the deep-Sea derived fungus Phomopsis lithocarpus FS508. Mar. Drugs 2018, 16, 329. [CrossRef]

71. Ma, K.X.; Shen, X.T.; Huang, R.; Wang, T.; Xie, X.S.; Liu, S.W.; Wu, S.H.; He, J. Bioactiye metabolites produced by the endophytic fungus Phomopsis sp. YM355364. Nat. Prod. Commun. 2014, 9, 669-670. [CrossRef] [PubMed]

72. Sheng, S.L.; Li, Y.P.; Xiang, H.Y.; Liu, Y.; Wang, Y.D.; Kong, L.P.; Du, G.; Hu, Q.F.; Chen, Y.J.; Wang, W.G. Histone deacetylase inhibitor induced lipase Inhibitors from endophytic Phomopsis sp. 0391. Rec. Nat. Prod. 2020, 14, 42-47. [CrossRef]

73. Kongprapan, T.; Xu, X.; Rukachaisirikul, V.; Phongpaichit, S.; Sakayaroj, J.; Chen, J.; Shen, X. Cytosporone derivatives from the endophytic fungus Phomopsis sp. PSU-H188. Phytochem. Lett. 2017, 22, 219-223. [CrossRef]

74. Xu, J.; Tan, H.; Chen, Y.; Li, S.; Guo, H.; Huang, Z.; Li, H.; Gao, X.; Liu, H.; Zhang, W. Lithocarpinols A and B, a pair of diastereomeric antineoplastic tenellone derivatives from the deep-sea derived fungus Phomopsis lithocarpus FS508. Chin. Chem. Lett. 2019, 30, 439-442. [CrossRef]

75. Chen, Y.G.; Pan, J.H.; Xu, F.; Liu, F.; Yang, J.X.; Huang, C.H.; Xu, C.L.; Lu, Y.J.; Cai, X.L.; She, Z.G.; et al. A new indene derivative from the marine fungus Phomopsis sp. (No. GX7-4A). Chem. Nat. Compd. 2010, 46, 230-232. [CrossRef] 
76. Tan, Q.W.; Fang, P.H.; Ni, J.C.; Gao, F.; Chen, Q.J. Metabolites Produced by an Endophytic Phomopsis sp. and Their Anti-TMV Activity. Molecules 2017, 22, 2073. [CrossRef]

77. Shiono, Y.; Muslihah, N.I.; Suzuki, T.; Ariefta, N.R.; Anwar, C.; Nurjanto, H.H.; Aboshi, T.; Murayama, T.; Tawaraya, K.; Koseki, T.; et al. New eremophilane and dichlororesorcinol derivatives produced by endophytes isolated from Ficus ampelas. J. Antibiot. 2017, 70, 1133-1137. [CrossRef]

78. Bunyapaiboonsri, T.; Yoiprommarat, S.; Srikitikulchai, P.; Srichomthong, K.; Lumyong, S. Oblongolides from the endophytic fungus Phomopsis sp. BCC 9789. J. Nat. Prod. 2010, 73, 55-59. [CrossRef]

79. Lin, T.; Wang, G.H.; Lin, X.; Hu, Z.Y.; Chen, Q.C.; Xu, Y.; Zhang, X.K.; Chen, H.F. Three new oblongolides from Phomopsis sp. XZ-01, an endophytic fungus from Camptotheca acuminate. Molecules 2011, 16, 3351-3359. [CrossRef]

80. Huang, R.; Jiang, B.G.; Li, X.N.; Wang, Y.T.; Liu, S.S.; Zheng, K.X.; He, J.; Wu, S.H. Polyoxygenated cyclohexenoids with promising $\alpha$-glycosidase inhibitory activity produced by Phomopsis sp. YE3250, an endophytic fungus derived from Paeonia delavayi. J. Agric. Food Chem. 2018, 66, 1140-1146. [CrossRef]

81. Cimmino, A.; Andolfi, A.; Zonno, M.C.; Troise, C.; Santini, A.; Tuzi, A.; Vurro, M.; Ash, G.; Evidente, A. Phomentrioloxin: A phytotoxic pentasubstituted geranylcyclohexentriol produced by Phomopsis sp., a potential mycoherbicide for Carthamus lanatus Biocontrol. J. Nat. Prod. 2012, 75, 1130-1137. [CrossRef] [PubMed]

82. Goddard, M.L.; Mottier, N.; Jeanneret-Gris, J.; Christen, D.; Tabacchi, R.; Abou-Mansour, E. Differential production of phytotoxins from Phomopsis sp. from grapevine plants showing esca symptoms. J. Agric. Food Chem. 2014, 62, 8602-8607. [CrossRef] [PubMed]

83. Xie, S.S.; Wu, Y.; Qiao, Y.B.; Guo, Y.; Wang, J.P.; Hu, Z.X.; Zhang, Q.; Li, X.N.; Huang, J.F.; Zhou, Q.; et al. Protoilludane, illudalane, and botryane sesquiterpenoids from the endophytic fungus Phomopsis sp. TJ507A. J. Nat. Prod. 2018, 81, 1311-1320. [CrossRef] [PubMed]

84. Zanardi, L.M.; Bolzani, V.d.S.; Cavalheiro, A.J.; Siqueira Silva, D.H.; Trevisan, H.C.; Araujo, A.R.; Silva, G.H.; Teles, H.L.; Young, M.C.M. Sesquiterpenes produced by endophytic fungus Phomopsis cassiae with antifungal and acetylcholinesterase inhibition activities. Quim. Nova 2012, 35, 2233-2236. [CrossRef]

85. Hemtasin, C.; Kanokmedhakul, S.; Kanokmedhakul, K.; Hahnvajanawong, C.; Soytong, K.; Prabpai, S.; Kongsaeree, P. Cytotoxic pentacyclic and tetracyclic aromatic sesquiterpenes from Phomopsis archeri. J. Nat. Prod. 2011, 74, 609-613. [CrossRef]

86. Ma, X.; Wang, W.; Li, E.; Gao, F.; Guo, L.; Pei, Y. A new sesquiterpene from the entomogenous fungus Phomopsis amygdali. Nat. Prod. Res. 2016, 30, 276-280. [CrossRef]

87. Qian, Y.X.; Kang, J.C.; Luo, Y.K.; He, J.; Wang, L.; Li, Q.R. Secondary metabolites of an endophytic fungus Phomopsis castaneaemollissimae. Chem. Nat. Compd. 2018, 54, 346-347. [CrossRef]

88. Wei, W.; Gao, J.; Shen, Y.; Chu, Y.L.; Xu, Q.; Tan, R.X. Immunosuppressive diterpenes from Phomopsis sp. S12. Eur. J. Org. Chem. 2014, 2014, 5728-5734. [CrossRef]

89. Xu, K.; Zhang, X.; Chen, J.W.; Shen, Y.; Jiang, N.; Tan, R.X.; Jiao, R.H.; Ge, H.M. Anti-inflammatory diterpenoids from an endophytic fungus Phomopsis sp. S12. Tetrahedron Lett. 2019, 60. [CrossRef]

90. Zhang, Y.; Hao, F.; Liu, N.; Xu, Y.; Jia, A.; Yang, Z.; Xia, X.; Liu, C. Stereochemical determination of a new and cytotoxic euphane triterpenoid from the plant endophytic fungus Phomopsis chimonanthi. J. Antibiot. 2013, 66, 679-682. [CrossRef]

91. Peyrat, L.A.; Eparvier, V.; Eydoux, C.; Guillemot, J.C.; Litaudon, M.; Stien, D. Betulinic acid, the first lupane-type triterpenoid isolated from both a Phomopsis sp. and its host plant Diospyros carbonaria benoist. Chem. Biodivers. 2017, 14. [CrossRef] [PubMed]

92. Wu, S.H.; Huang, R.; Miao, C.P.; Chen, Y.W. Two new steroids from an endophytic fungus Phomopsis sp. Chem. Biodivers. 2013, 10, 1276-1283. [CrossRef] [PubMed]

93. Hu, Z.X.; Wu, Y.; Xie, S.S.; Sun, W.G.; Guo, Y.; Li, X.N.; Liu, J.J.; Li, H.; Wang, J.P.; Luo, Z.W.; et al. Phomopsterones A and B, two functionalized ergostane-type steroids from the endophytic fungus Phomopsis sp. TJ507A. Org. Lett. 2017, 19, $258-261$. [CrossRef] [PubMed]

94. Adelin, E.; Servy, C.; Cortial, S.; Levaique, H.; Martin, M.T.; Retailleau, P.; Le Goff, G.; Bussaban, B.; Lumyong, S.; Ouazzani, J. Isolation, structure elucidation and biological activity of metabolites from Sch-642305-producing endophytic fungus Phomopsis sp. CMU-LMA. Phytochemistry 2011, 72, 2406-2412. [CrossRef] [PubMed]

95. Xu, J.; Tan, H.; Chen, Y.; Li, S.; Huang, Z.; Guo, H.; Li, H.; Gao, X.; Liu, H.; Zhang, W. Lithocarpins A-D: Four tenellone-macrolide conjugated [ $4+2]$ hetero-adducts from the deep-sea derived fungus Phomopsis lithocarpus FS508. Org. Chem. Front. 2018, 5, 1792-1797. [CrossRef]

96. Yan, B.C.; Wang, W.G.; Hu, D.B.; Sun, X.; Kong, L.M.; Li, X.N.; Du, X.; Luo, S.H.; Liu, Y.; Li, Y.; et al. Phomopchalasins A and B, two cytochalasans with polycyclic-fused skeletons from the endophytic fungus Phomopsis sp. shj2. Org. Lett. 2016, 18, 1108-1111. [CrossRef]

97. Luo, Y.F.; Zhang, M.; Dai, J.G.; Pedpradab, P.; Wang, W.J.; Wu, J. Cytochalasins from mangrove endophytic fungi Phomopsis spp. xy21 and xy22. Phytochem. Lett. 2016, 17, 162-166. [CrossRef]

98. Jouda, J.B.; Tamokou, J.D.D.; Mbazoa, C.D.; Douala-Meli, C.; Sarkar, P.; Bag, P.K.; Wandji, J. Antibacterial and cytotoxic cytochalasins from the endophytic fungus Phomopsis sp. harbored in Garcinia kola (Heckel) nut. BMC Complement. Altern. Med. 2016, 16. [CrossRef]

99. Fu, J.; Zhou, Y.; Li, H.F.; Ye, Y.H.; Guo, J.H. Antifungal metabolites from Phomopsis sp. By254, an endophytic fungus in Gossypium hirsutum. Afr. J. Microbiol. Res. 2011, 5, 1231-1236. [CrossRef] 
100. Chang, H.S.; Peng, C.J.; Cheng, M.J.; Wu, H.C.; Chan, H.Y.; Hsieh, S.Y.; Yuan, G.F.; Chen, I.S. Chemical constituents of the endophytic fungus Phomopsis asparagi isolated from the plant Peperomia sui. Chem. Nat. Compd. 2018, 54, 504-508. [CrossRef]

101. Chen, S.C.; Liu, Z.M.; Tan, H.B.; Chen, Y.C.; Li, S.N.; Li, H.H.; Guo, H.; Zhu, S.; Liu, H.X.; Zhang, W.M. Tersone A-G, new pyridone alkaloids from the deep-sea fungus Phomopsis tersa. Mar. Drugs 2019, 17, 394. [CrossRef] [PubMed]

102. Chen, H.; Huang, M.; Li, X.; Liu, L.; Chen, B.; Wang, J.; Lin, Y. Phochrodines A-D, first naturally occurring new chromenopyridines from mangrove entophytic fungus Phomopsis sp. 33\#. Fitoterapia 2018, 124, 103-107. [CrossRef] [PubMed]

103. Verekar, S.A.; Mishra, P.D.; Sreekumar, E.S.; Deshmukh, S.K.; Fiebig, H.H.; Kelter, G.; Maier, A. Anticancer activity of new depsipeptide compound isolated from an endophytic fungus. J. Antibiot. 2014, 67, 697-701. [CrossRef] [PubMed]

104. Katz, L. Manipulation of modular polyketide syntheses. Chem. Rev. 1997, 97, 2557-2575. [CrossRef]

105. Le Pogam, P.; Boustie, J. Xanthones of lichen source: A 2016 update. Molecules 2016, 21, 294. [CrossRef]

106. Duan, Y.D.; Jiang, Y.Y.; Guo, F.X.; Chen, L.X.; Xu, L.L.; Zhang, W.; Liu, B. The antitumor activity of naturally occurring chromones: A review. Fitoterapia 2019, 135, 114-129. [CrossRef]

107. Mayuri, B.; Kavitha, P.; Basavoju, S.; Bhargavi, G.; Reddy, K.L. Synthesis, structural characterisation and biological evolution of chromanones. J. Mol. Struct. 2017, 1145, 1-9. [CrossRef]

108. Li, Y.; Li, X.; Cheng, J.P. Catalytic asymmetric synthesis of chiral benzofuranones. Adv. Synth. Catal. 2014, 356, 1172-1198. [CrossRef]

109. McGlacken, G.P.; Fairlamb, I.J.S. 2-Pyrone natural products and mimetics: Isolation, characterisation and biological activity. Nat Prod. Rep. 2005, 22, 369-385. [CrossRef]

110. Bolton, J.L.; Dunlap, T. Formation and biological targets of quinones: Cytotoxic versus cytoprotective effects. Chem. Res. Toxicol. 2017, 30, 13-37. [CrossRef]

111. Jones, T.J.M.; Douglas, C.J. The metabolism and toxicity of quinones, quinonimines, quinone methides, and quinone-thioethers. Curr. Drug Metab. 2002, 3, 425-438. [CrossRef]

112. Gajera, H.P.; Gevariya, S.N.; Hirpara, D.G.; Patel, S.V.; Golakiya, B.A. Antidiabetic and antioxidant functionality associated with phenolic constituents from fruit parts of indigenous black jamun (Syzygium cumini L.) landraces. J. Food Sci. Technol. 2017, 54, 3180-3191. [CrossRef] [PubMed]

113. Shing, T.K.M.; Yang, J. A short synthesis of natural (-)-oblongolide via an intramolecular or a transannular diels-alder reaction. J. Org. Chem. 1995, 60, 5785-5789. [CrossRef]

114. Huang, M.; Lu, J.J.; Huang, M.Q.; Bao, J.L.; Chen, X.P.; Wang, Y.T. Terpenoids: Natural products for cancer therapy. Expert Opin. Investig. Drugs 2012, 21, 1801-1818. [CrossRef] [PubMed]

115. Thoppil, R.J.; Bishayee, A. Terpenoids as potential chemopreventive and therapeutic agents in liver cancer. World J. Hepatol. 2011, 3, 228-249. [CrossRef]

116. Zielinska-Blajet, M.; Feder-Kubis, J. Monoterpenes and their derivatives-recent development in biological and medical applications. Int. J. Mol. Sci. 2020, 21, 7078. [CrossRef]

117. Chen, D.L.; Wang, B.W.; Sun, Z.C.; Yang, J.S.; Xu, X.D.; Ma, G.X. Natural nitrogenous sesquiterpenoids and their bioactivity: A review. Molecules 2020, 25, 2485. [CrossRef]

118. Chen, L.; Lu, X.; El-Seedi, H.; Teng, H. Recent advances in the development of sesquiterpenoids in the treatment of type 2 diabetes. Trends Food Sci. Technol. 2019, 88, 46-56. [CrossRef]

119. Su, Y.D.; Su, J.H.; Hwang, T.L.; Wen, Z.H.; Sheu, J.H.; Wu, Y.C.; Sung, P.J. Briarane diterpenoids isolated from octocorals between 2014 and 2016. Mar. Drugs 2017, 15, 44. [CrossRef]

120. Ren, Y.; Kinghorn, A.D. Natural product triterpenoids and their semi-synthetic derivatives with potential anticancer activity. Planta Med. 2019, 85, 802-814. [CrossRef]

121. Rahman, S.U.; Ismail, M.; Khurram, M.; Ullah, I.; Rabbi, F.; Iriti, M. Bioactive steroids and saponins of the genus Trillium. Molecules 2017, 22, 2156. [CrossRef] [PubMed]

122. Wang, M.; Zhang, J.; He, S.; Yan, X. A review study on macrolides isolated from cyanobacteria. Mar. Drugs 2017, 15, 126. [CrossRef] [PubMed]

123. Mishra, S.K.; Tripathi, G.; Kishore, N.; Singh, R.K.; Singh, A.; Tiwari, V.K. Drug development against tuberculosis: Impact of alkaloids. Eur. J. Med. Chem. 2017, 137, 504-544. [CrossRef] [PubMed]

124. Wang, T.Y.; Li, Q.; Bi, K.S. Bioactive flavonoids in medicinal plants: Structure, activity and biological fate. Asian J. Pharm. 2018, 13, 12-23. [CrossRef]

125. Luo, X.; Yang, J.; Chen, F.; Lin, X.; Chen, C.; Zhou, X.; Liu, S.; Liu, Y. Structurally diverse polyketides from the mangrove-derived fungus Diaporthe sp. SCSIO 41011 with their anti-influenza A virus activities. Front. Chem. 2018, 6. [CrossRef]

126. Liu, Y.; Ruan, Q.; Jiang, S.; Qu, Y.; Chen, J.; Zhao, M.; Yang, B.; Liu, Y.; Zhao, Z.; Cui, H. Cytochalasins and polyketides from the fungus Diaporthe sp. GZU-1021 and their anti-inflammatory activity. Fitoterapia 2019, 137. [CrossRef]

127. Niu, Z.; Chen, Y.; Guo, H.; Li, S.N.; Li, H.H.; Liu, H.X.; Liu, Z.; Zhang, W. Cytotoxic polyketides from a deep-sea sediment derived fungus Diaporthe phaseolorum FS431. Molecules 2019, 24, 3062. [CrossRef]

128. Cui, H.; Ding, M.; Huang, D.; Zhang, Z.; Liu, H.; Huang, H.; She, Z. Chroman-4-one and pyrano[4,3-b]chromenone derivatives from the mangrove endophytic fungus Diaporthe phaseolorum SKS019. RSC Adv. 2017, 7, 20128-20134. [CrossRef]

129. Liu, Z.; Zhao, J.; Liang, X.; Lv, X.; Li, Y.; Qu, J.; Liu, Y. Dothiorelone derivatives from an endophyte Diaporthe pseudomangiferaea inhibit the activation of human lung fibroblasts MRC-5 cells. Fitoterapia 2018, 127, 7-14. [CrossRef] 
130. Bungihan, M.E.; Tan, M.A.; Kitajima, M.; Kogure, N.; Franzblau, S.G.; dela Cruz, T.E.E.; Takayama, H.; Nonato, M.G. Bioactive metabolites of Diaporthe sp. P133, an endophytic fungus isolated from Pandanus amaryllifolius. J. Nat. Med. 2011, 65, 606-609. [CrossRef]

131. De Medeiros, A.G.; Savi, D.C.; Mitra, P.; Shaaban, K.A.; Jha, A.K.; Thorson, J.S.; Rohr, J.; Glienke, C. Bioprospecting of Diaporthe terebinthifolii LGMF907 for antimicrobial compounds. Folia Microbiol. 2018, 63, 499-505. [CrossRef] [PubMed]

132. Meepagala, K.M.; Briscoe, W.E.; Techen, N.; Johnson, R.D.; Clausen, B.M.; Duke, S.O. Isolation of a phytotoxic isocoumarin from Diaporthe eres-infected Hedera helix (English ivy) and synthesis of its phytotoxic analogs. Pest Manag. Sci. 2018, 74, 37-45. [CrossRef] [PubMed]

133. Sharma, V.; Singamaneni, V.; Sharma, N.; Kumar, A.; Arora, D.; Kushwaha, M.; Bhushan, S.; Jaglan, S.; Gupta, P. Valproic acid induces three novel cytotoxic secondary metabolites in Diaporthe sp., an endophytic fungus from Datura inoxia Mill. Bioorganic Med. Chem. Lett. 2018, 28, 2217-2221. [CrossRef] [PubMed]

134. Liu, Y.; Hu, Z.; Lin, X.; Lu, C.; Shen, Y. A new polyketide from Diaporthe sp. SXZ-19, an endophytic fungal strain of Camptotheca acuminate. Nat. Prod. Res. 2013, 27, 2100-2104. [CrossRef] [PubMed]

135. Evidente, M.; Boari, A.; Vergura, S.; Cimmino, A.; Vurro, M.; Ash, G.; Superchi, S.; Evidente, A. Structure and absolute configuration of kongiidiazadione, a new phytotoxic 3-substituted-5-diazenylcyclopentendione produced by Diaporthe Kongii. Chirality 2015, 27, 557-562. [CrossRef]

136. Tanney, J.B.; McMullin, D.R.; Green, B.D.; Miller, J.D.; Seifert, K.A. Production of antifungal and antiinsectan metabolites by the Picea endophyte Diaporthe maritima sp. nov. Fungal Biol. 2016, 120, 1448-1457. [CrossRef]

137. Riga, R.; Happyana, N.; Quentmeier, A.; Zammarelli, C.; Kayser, O.; Hakim, E.H. Secondary metabolites from Diaporthe lithocarpus isolated from Artocarpus heterophyllus. Nat. Prod. Res. 2019. [CrossRef]

138. Ratnaweera, P.B.; Jayasundara, J.M.N.M.; Herath, H.H.M.S.D.; Williams, D.E.; Rajapaksha, S.U.; Nishantha, K.M.D.W.P.; de Silva, E.D.; Andersen, R.J. Antifeedant, contact toxicity and oviposition deterrent effects of phyllostine acetate and phyllostine isolated from the endophytic fungus Diaporthe miriciae against Plutella xylostella larvae. Pest Manag. Sci. 2020, 76, 1541-1548. [CrossRef]

139. Wulansari, D.; Julistiono, H.; Nurkanto, A.; Agusta, A. Antifungal activity of (+)-2,2'-epicytoskyrin A and its membrane-disruptive action. Makara J. Sci. 2016, 20, 160-166. [CrossRef]

140. Tian, W.; Liao, Z.; Zhou, M.; Wang, G.; Wu, Y.; Gao, S.; Qiu, D.; Liu, X.; Lin, T.; Chen, H. Cytoskyrin C, an unusual asymmetric bisanthraquinone with cage-like skeleton from the endophytic fungus Diaporthe sp. Fitoterapia 2018, 128, 253-257. [CrossRef]

141. Specian, V.; Sarragiotto, M.H.; Pamphile, J.A.; Clemente, E. Chemical characterization of bioactive compounds from the endophytic fungus Diaporthe helianthi isolated from Luehea divaricata. Braz. J. Microbiol. 2012, 43, 1174-1182. [CrossRef] [PubMed]

142. Reveglia, P.; Pacetti, A.; Masi, M.; Cimmino, A.; Carella, G.; Marchi, G.; Mugnai, L.; Evidente, A. Phytotoxic metabolites produced by Diaporthe eres involved in cane blight of grapevine in Italy. Nat. Prod. Res. 2019. [CrossRef] [PubMed]

143. Noriler, S.A.; Savi, D.C.; Ponomareva, L.V.; Rodrigues, R.; Rohr, J.; Thorson, J.S.; Glienke, C.; Shaaban, K.A. Vochysiamides A and B: Two new bioactive carboxamides produced by the new species Diaporthe vochysiae. Fitoterapia 2019, 138. [CrossRef] [PubMed]

144. Sousa, J.P.B.; Aguilar-Perez, M.M.; Arnold, A.E.; Rios, N.; Coley, P.D.; Kursar, T.A.; Cubilla-Rios, L. Chemical constituents and their antibacterial activity from the tropical endophytic fungus Diaporthe sp. F2934. J. Appl. Microbiol. 2016, 120, 1501-1508. [CrossRef]

145. Ola, A.R.B.; Debbab, A.; Kurtan, T.; Broetz-Oesterhelt, H.; Aly, A.H.; Proksch, P. Dihydroanthracenone metabolites from the endophytic fungus Diaporthe melonis isolated from Annona squamosa. Tetrahedron Lett. 2014, 55, 3147-3150. [CrossRef]

146. Nakashima, K.I.; Tomida, J.; Kamiya, T.; Hirai, T.; Morita, Y.; Hara, H.; Kawamura, Y.; Adachi, T.; Inoue, M. Diaporthols A and B: Bioactive diphenyl ether derivatives from an endophytic fungus Diaporthe sp. Tetrahedron Lett. 2018, 59, 1212-1215. [CrossRef]

147. Cui, H.; Lin, Y.; Luo, M.; Lu, Y.; Huang, X.; She, Z. Diaporisoindoles A-C: Three isoprenylisoindole alkaloid derivatives from the mangrove endophytic fungus Diaporthe sp. SYSU-HQ3. Org. Lett. 2017, 19, 5621-5624. [CrossRef]

148. Cui, H.; Liu, Y.; Li, J.; Huang, X.; Yan, T.; Cao, W.; Liu, H.; Long, Y.; She, Z. Diaporindenes A-D: Four unusual 2,3-dihydro-1Hindene analogues with anti-inflammatory activities from the mangrove endophytic fungus Diaporthe sp. SYSU-HQ3. J. Org. Chem. 2018, 83, 11804-11813. [CrossRef]

149. Andolfi, A.; Boari, A.; Evidente, M.; Cimmino, A.; Vurro, M.; Ash, G.; Evidente, A. Gulypyrones A and B and phomentrioloxins B and C Produced by Diaporthe gulyae, a potential mycoherbicide for saffron thistle (Carthamus lanatus). J. Nat. Prod. 2015, 78, 623-629. [CrossRef]

150. Luo, X.; Lin, X.; Tao, H.; Wang, J.; Li, J.; Yang, B.; Zhou, X.; Liu, Y. Isochromophilones A-F, cytotoxic chloroazaphilones from the marine mangrove endophytic fungus Diaporthe sp. SCSIO 41011. J. Nat. Prod. 2018, 81, 934-941. [CrossRef]

151. Chen, C.J.; Liu, X.X.; Zhang, W.J.; Zang, L.Y.; Wang, G.; Ng, S.W.; Tan, R.X.; Ge, H.M. Sesquiterpenoids isolated from an endophyte fungus Diaporthe sp. RSC Adv. 2015, 5, 17559-17565. [CrossRef]

152. Mandavid, H.; Rodrigues, A.M.S.; Espindola, L.S.; Eparvier, V.; Stien, D. Secondary metabolites isolated from the amazonian endophytic fungus Diaporthe sp. SNB-GSS10. J. Nat. Prod. 2015, 78, 1735-1739. [CrossRef] [PubMed]

153. Liu, H.; Chen, Y.; Li, H.; Li, S.; Tan, H.; Liu, Z.; Li, D.; Liu, H.; Zhang, W. Four new metabolites from the endophytic fungus Diaporthe lithocarpus A740. Fitoterapia 2019, 137. [CrossRef] [PubMed]

154. Li, G.; Kusari, S.; Kusari, P.; Kayser, O.; Spiteller, M. Endophytic Diaporthe sp. LG23 produces a potent antibacterial tetracyclic triterpenoid. J. Nat. Prod. 2015, 78, 2128-2132. [CrossRef] 
155. Ito, A.; Maeda, H.; Tonouchi, A.; Hashimoto, M. Relative and absolute structure of phomolide C. Biosci. Biotechnol. Biochem. 2015, 79, 1067-1069. [CrossRef]

156. Yedukondalu, N.; Arora, P.; Wadhwa, B.; Malik, F.A.; Vishwakarma, R.A.; Gupta, V.K.; Riyaz-Ul-Hassan, S.; Ali, A. Diapolic acid A-B from an endophytic fungus, Diaporthe terebinthifolii depicting antimicrobial and cytotoxic activity. J. Antibiot. 2017, 70, 212-215. [CrossRef]

157. Brissow, E.R.; da Silva, I.P.; de Siqueira, K.A.; Senabio, J.A.; Pimenta, L.P.; Januario, A.H.; Magalhaes, L.G.; Furtado, R.A.; Tavares, D.C.; Sales Junior, P.A.; et al. 18-Des-hydroxy cytochalasin: An antiparasitic compound of Diaporthe phaseolorum-92C, an endophytic fungus isolated from Combretum lanceolatum Pohl ex Eichler. Parasitol. Res. 2017, 116, 1823-1830. [CrossRef]

158. Huang, X.; Zhou, D.; Liang, Y.; Liu, X.; Cao, F.; Qin, Y.; Mo, T.; Xu, Z.; Li, J.; Yang, R. Cytochalasins from endophytic Diaporthe sp. GDG-118. Nat. Prod. Res. 2019. [CrossRef]

159. Chang, F.R.; Wang, S.W.; Li, C.Y.; Lu, Y.Y.; Liu, S.Y.V.; Chen, C.Y.; Wu, Y.C.; Cheng, Y.B. Natural products from Diaporthe arecae with anti-angiogenic activity. Isr. J. Chem. 2019, 59, 439-445. [CrossRef]

160. Cui, H.; Yu, J.; Chen, S.; Ding, M.; Huang, X.; Yuan, J.; She, Z. Alkaloids from the mangrove endophytic fungus Diaporthe phaseolorum SKS019. Bioorganic Med. Chem. Lett. 2017, 27, 803-807. [CrossRef]

161. Schloss, S.; Hackl, T.; Herz, C.; Lamy, E.; Koch, M.; Rohn, S.; Maul, R. Detection of a toxic methylated derivative of phomopsin A produced by the legume-infesting fungus Diaporthe toxica. J. Nat. Prod. 2017, 80, 1930-1934. [CrossRef] [PubMed]

162. Sebastianes, F.L.S.; Cabedo, N.; El Aouad, N.; Valente, A.M.M.P.; Lacava, P.T.; Azevedo, J.L.; Pizzirani-Kleiner, A.A.; Cortes, D. 3-Hydroxypropionic acid as an antibacterial agent from endophytic fungi Diaporthe phaseolorum. Curr. Microbiol. 2012, 65, 622-632. [CrossRef] [PubMed]

163. Hu, M.; Yang, X.Q.; Wan, C.P.; Wang, B.Y.; Yin, H.Y.; Shi, L.J.; Wu, Y.M.; Yang, Y.B.; Zhou, H.; Ding, Z.T. Potential antihyperlipidemic polyketones from endophytic Diaporthe sp. JC-J7 in Dendrobium nobile. RSC Adv. 2018, 8, 41810-41817. [CrossRef]

164. Yenn, T.W.; Ring, L.C.; Nee, T.W.; Khairuddean, M.; Zakaria, L.; Ibrahim, D. Endophytic Diaporthe sp. ED2 produces a novel anti-candidal ketone derivative. J. Microbiol. Biotechnol. 2017, 27, 1065-1070. [CrossRef]

165. El-Helw, E.A.E.; Hashem, A.I. Synthesis and antitumor activity evaluation of some pyrrolone and pyridazinone heterocycles derived from 3-((2-oxo-5-(p-tolyl)furan-3(2H)-ylidene)methyl)quinolin-2(1H)-one. Synth. Commun. 2020, 50, $1046-1055$. [CrossRef]

166. Zheng, C.J.; Shao, C.L.; Chen, M.; Niu, Z.G.; Zhao, D.L.; Wang, C.Y. Merosesquiterpenoids and ten-membered macrolides from a soft coral-derived Lophiostoma sp. fungus. Chem. Biodivers. 2015, 12, 1407-1414. [CrossRef]

167. Jozwiak, M.; Filipowska, A.; Fiorino, F.; Struga, M. Anticancer activities of fatty acids and their heterocyclic derivatives. Eur. J. Pharmacol. 2020, 871. [CrossRef] 